\title{
C-GOALS: Chandra observations of a complete sample of luminous infrared galaxies from the IRAS Revised Bright Galaxy Survey
}

\author{
K. Iwasawa ${ }^{1}$, D. B. Sanders ${ }^{2}$, S. H. Teng ${ }^{3}$, V. U ${ }^{2,4, \star}$, L. Armus ${ }^{5}$, A. S. Evans ${ }^{6}$, J. H. Howell ${ }^{5}$, S. Komossa ${ }^{7}$, \\ J. M. Mazzarella ${ }^{8}$, A. O. Petric ${ }^{5}$, J. A. Surace ${ }^{5}$, T. Vavilkin ${ }^{9}$, S. Veilleux ${ }^{3}$, and N. Trentham ${ }^{10}$ \\ 1 ICREA and Institut de Ciències del Cosmos (ICC), Universitat de Barcelona (IEEC-UB), Martí i Franquès, 1, 08028 Barcelona, \\ Spain \\ e-mail: kazushi.iwasawa@icc.ub.edu \\ 2 Institute for Astronomy, 2680 Woodlawn Drive, Honolulu, Hawaii 96822, USA \\ 3 Department of Astronomy, University of Maryland, College Park, MD 20742, USA \\ 4 SAO Predoctoral Fellow, Harvard-Smithsonian Center for Astrophysics, Cambridge, MA 02138, USA \\ 5 Spitzer Science Center, California Institute of Technology, Pasadena, CA 91125, USA \\ 6 Department of Astronomy, University of Virginia, 530 McCormick Road, Charlottesville, VA 22904 and NRAO, \\ 520 Edgemont Road, Charlottesville, VA 22903, USA \\ 7 Max Planck Institut für extraterrestrische Physik, Gießenbachstraße, 85748 Garching, Germany \\ 8 IPAC, California Institute of Technology, Pasadena, CA 91125, USA \\ 9 Department of Physics and Astronomy, State University of New York at Stony Brook, NY 11794, USA \\ 10 Institute of Astronomy, Madingley Road, Cambridge CB3 OHA, UK
}

Received 23 June 2010 / Accepted 25 February 2011

\section{ABSTRACT}

\begin{abstract}
We present X-ray data for a complete sample of 44 luminous infrared galaxies (LIRGs), obtained with the Chandra X-ray Observatory. These are the X-ray observations of the high luminosity portion of the Great Observatory All-sky LIRG Survey (GOALS), which includes the most luminous infrared selected galaxies, $\log \left(L_{\mathrm{ir}} / L_{\odot}\right) \geq 11.73$, in the local universe, $z \leq 0.088$. X-rays were detected from 43 out of 44 objects, and their arcsec-resolution images, spectra, and radial brightness distributions are presented. With a selection by hard X-ray colour and the $6.4 \mathrm{keV}$ iron line, AGN are found in $37 \%$ of the objects, with higher luminosity sources more likely to contain an AGN. These AGN also tend to be found in late-stage mergers. The AGN fraction would increase to $48 \%$ if objects with [Ne v] $\lambda 14.3 \mu \mathrm{m}$ detection are included. Double AGN are clearly detected only in NGC 6240 among 24 double/triple systems. Other AGN are found either in single nucleus objects or in one of the double nuclei at similar rates. Objects without conventional $\mathrm{X}$-ray signatures of AGN appear to be hard X-ray quiet, relative to the X-ray to far-IR correlation for starburst galaxies, as discussed elsewhere. Most objects also show extended soft X-ray emission, which is likely related to an outflow from the nuclear region, with a metal abundance pattern suggesting enrichment by core collapse supernovae, as expected for a starburst.
\end{abstract}

Key words. infrared: galaxies - X-rays: galaxies - galaxies: active - galaxies: starburst

\section{Introduction}

The Great Observatory All-sky LIRGs Survey (GOALS, Armus et al. 2009) is a multi-wavelength study of the most luminous infrared galaxies in the local Universe, selected from the $60 \mu \mathrm{m}$ flux limited IRAS Revised Bright Galaxy Sample (RBGS: Sanders et al. 2003). As the brightest far-infrared selected galaxies in the sky, these objects are the most amenable for study at all wavelengths.

Luminous infrared galaxies (LIRGs: $L_{\mathrm{ir}}>10^{11} L_{\odot}{ }^{1}$ ) have proven to be an extremely important class of extragalactic objects. In the local Universe they are more numerous than optically selected starburst and Seyfert galaxies and quasi-stellar objects (QSOs) at comparable bolometric luminosity. Strong interactions and mergers of gas-rich spirals appear to be the trigger for the most luminous infrared objects, which are fueled by a mixture of intense starbursts and AGN, with the latter becoming

\footnotetext{
* NASA Jenkins Predoctoral Fellow.

${ }^{1} L_{\text {ir }} \equiv L(8-1000 \mu \mathrm{m})$.
}

more dominant with increasing $L_{\mathrm{ir}}$. At the highest luminosities, ultraluminous infrared galaxies (ULIRGs: $L_{\mathrm{ir}}>10^{12} L_{\odot}$ ), may represent an important stage in the formation of QSOs and powerful radio galaxies, and they may also represent a primary stage in the formation of massive ellipticals, the formation of globular clusters, and the metal enrichment of the intergalactic medium (see Sanders \& Mirabel 1996, for a more complete review and, e.g., Chapman et al. 2005; Hopkins et al. 2006; Veilleux et al. 2009 , for recent developments).

The X-ray survey data presented here (C-GOALS) is the $\mathrm{X}$-ray component of the GOALS multi-wavelength survey. This initial C-GOALS paper presents data obtained by us and others with the Chandra X-ray Observatory (Chandra, hereafter). Data obtained by us in Cycles $7+8$ have been combined with data from the Chandra Archive to produce the first complete X-ray survey of the most luminous sources in the GOALS sample. Previous X-ray investigations of LIRGs, either by Chandra, XMM-Newton or Suzaku, have been presented in Ptak et al. (2003), Franceschini et al. (2003), Teng et al. (2005, 2009), and Grimes et al. (2005). 


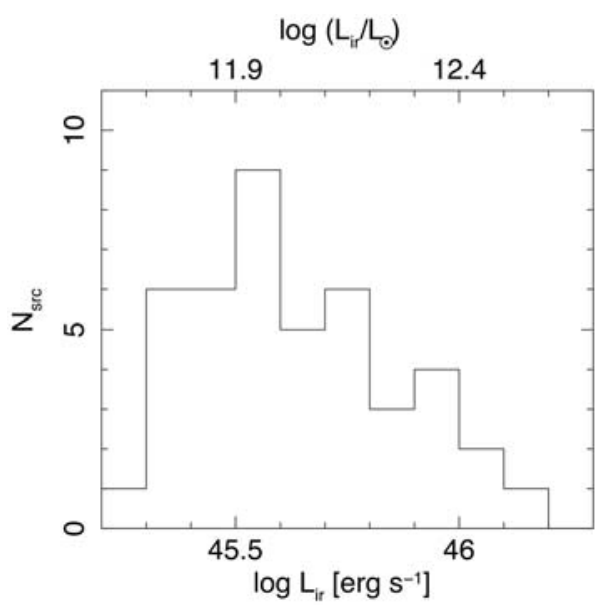

Fig. 1. The distribution of infrared luminosity, $L_{\text {ir }}(8-1000 \mu \mathrm{m})$ for the 44 objects in our C-GOALS sample with $\log \left(L_{\text {ir }} / L_{\odot}\right)=11.73-12.57$. For the infrared luminosity, we have chosen to use units of $\mathrm{erg} \mathrm{s}^{-1}$ (bottom axis), rather than the unit of $L_{\odot}$ (top axis) normally used in infrared astronomy, in order to be consistent with X-ray luminosity measurements.

The C-GOALS sample and observations are described in Sects. 2 and 3, respectively, with the major results (X-ray images, flux density spectra, radial surface brightness profiles) presented in Sect. 4. Derived properties from the X-ray spectra, and a discussion of trends of X-ray properties with infrared luminosity are presented in Sect. 5. A summary of our results is given in Sect. 6. Notes for each object are presented in the Appendix. The cosmology adopted here is consistent with that adopted by Armus et al. (2009). Cosmological distances were computed by first correcting for the 3-attractor flow model of Mould et al. (2000) and adopting $H_{0}=70 \mathrm{~km} \mathrm{~s}^{-1} \mathrm{Mpc}^{-1}, \Omega_{\mathrm{V}}=0.72$, and $\Omega_{\mathrm{M}}=0.28$ based on the 5-year WMAP results (Hinshaw et al. 2009), as provided by the NASA/IPAC Extragalactic Database (NED).

\section{The sample}

The RBGS is a complete sample of 629 extragalactic objects with IRAS $60 \mu \mathrm{m}$ flux density greater than $5.24 \mathrm{Jy}$, covering the full sky above Galactic latitude $|b|>5^{\circ}$. The GOALS sample contains 202 "systems" (77 of which contain multiple galaxies), drawn from the RBGS with the luminosity threshold $L_{\mathrm{ir}} \geq$ $10^{11} L_{\odot}$ (see Armus et al. 2009, for further details). The current C-GOALS sample represents the high luminosity part of the GOALS sample, and is complete down to $\log \left(L_{\text {ir }} / L_{\odot}\right)=11.73$. The object with the largest IR luminosity, $\log \left(L_{\text {ir }} / L_{\odot}\right)=12.57$, is UGC 8058 (hereafter the more common name, Mrk 231, is used in the text). Table 1 gives basic data for all 44 galaxies in the C-GOALS sample. The redshift range is $z=0.010-0.088$. The median IR luminosity of the sample $\log \left(L_{\text {ir }} / L_{\odot}\right)=11.99$ corresponding to $\log L_{\mathrm{ir}}=45.58\left(\mathrm{erg} \mathrm{s}^{-1}\right)$. The distribution of infrared luminosity is shown in Fig. 1.

\section{Observations and data reduction}

Twenty-six objects were observed with Chandra in Cycle-8 (PI: D. Sanders) with a uniform $15 \mathrm{ks}$ exposure on each target. All the observations were carried out in imaging mode with the ACIS$\mathrm{S}$ detector operated in VFAINT mode. The Chandra data for the remaining 18 targets were taken from the Archive, including four galaxies in the Cycle 7 mini survey of LIRGs with double nuclei (UGC 4881, VV 705, F08572+3915, and F14348-1447) (PI: S Komossa). The exposure times for these 4 objects are also $15 \mathrm{ks}$ each. For other archival data, the exposure times vary from $10 \mathrm{ks}$ to $154 \mathrm{ks}$. The observation log for the 44 galaxies is shown in Table 2. Where multiple Chandra observations are found, an imaging observation with the ACIS-S is chosen. For Mrk 231, four ACIS-S observations exist in the archive, and we use all four to construct and analyze the X-ray images, while only the first observation was used for the analysis of the radial surface brightness profiles and the X-ray colour, since the source is not variable and the single observation provides good quality data for this purpose.

In Table 2, the Galactic HI column density, estimated from the Leiden/Argentine/Bonn (LAB) survey (Kalberla et al. 2005), is also quoted. Some of the Cycle- 8 targets lie at low Galactic latitudes and thus have been little studied because of high Galactic extinction. The Galactic HI absorption column exceeds $10^{21} \mathrm{~cm}^{-2}$ for six objects.

The data reduction was performed using the Chandra data analysis package CIAO version 3.4.1, and HEASARC's FTOOLS.

\section{Results}

Basic X-ray properties obtained from the Chandra data are presented in Table 3. For each object, the ACIS-S count rates in the soft $(0.5-2 \mathrm{keV})$ and hard $(2-7 \mathrm{keV})$ bands, the X-ray colour, estimated X-ray fluxes, X-ray luminosities in the two bands, and their logarithmic ratio to the infrared luminosity, $L_{\mathrm{ir}}(8-1000 \mu \mathrm{m})$, are listed. The count rates are computed for spatially distinctive components within a single object when they are clearly resolved, as opposed to the source counts in Table 2, which were collected from the whole X-ray emitting region per object. Similarly, IR luminosity is divided into respective components, estimated from IRAS HIRES processing (Surace et al. 2004) and new estimates based on Spitzer MIPS photometry (see Howell et al. 2010). The X-ray colour is defined as $H R=(H-S) /(H+S)$, often referred as the "Hardness Ratio", where $H$ and $S$ are background-corrected counts in the $2-8 \mathrm{keV}$ and $0.5-2 \mathrm{keV}$ bands, respectively.

We use the energy ranges, particularly for the hard X-ray band, that are slightly different from one another, but optimized for different purposes, which can sometimes be confusing. Here, we summarize the choice of the energy ranges used in this paper. 1) $0.4-7 \mathrm{keV}$ : this is effectively the full ACIS-S bandpass, and used only for observed counts; 2) $0.5-2 \mathrm{keV}$ : the soft X-ray band is always this range; 3 ) $2-8 \mathrm{keV}$ : the choice of this band is to match the conventional calculation of hardness ratio, $H R$, and used only for the hard band counts in Table 3 . The quoted counts are as observed, although the majority of our sources have negligible counts above $7 \mathrm{keV}$ where the background would simply increases; 4) 2-7 keV: this is the nominal range for our observed hard band flux, estimated from spectral fitting, and optimized for the signal to noise ratio of the data for the majority of our sources for the reason mentioned above; and 5) 2-10 keV: for the hard band luminosity, we opted to use this extended range for a purpose of comparison with results from other X-ray observatories, since the $2-10 \mathrm{keV}$ is the standard band for missions like XMM-Newton.

The images, spectra, colour and the selection of AGN based on the colour, the correlation of X-ray and infrared luminosities and the surface brightness distribution of individual objects are presented in the subsections below. 
Table 1. The C-GOALS sample.

\begin{tabular}{|c|c|c|c|c|c|c|c|c|}
\hline No. & IRAS Name & Optical ID & $\begin{array}{c}\text { RA (NED) } \\
\text { (J2000) } \\
(4)\end{array}$ & $\begin{array}{c}\text { Dec (NED) } \\
(\mathrm{J} 2000) \\
(5)\end{array}$ & $\begin{array}{c}z \\
\left(\mathrm{~km} \mathrm{~s}^{-1}\right) \\
(6)\end{array}$ & $\begin{array}{c}D_{\mathrm{L}} \\
(\mathrm{Mpc}) \\
(7)\end{array}$ & $\begin{array}{c}\log \left(L_{\text {ir }}\right) \\
\left(L_{\odot}\right) \\
(8)\end{array}$ & $\begin{array}{l}\text { Other name } \\
\text { (9) }\end{array}$ \\
\hline 1 & F01364-1042 & IRAS F01364-1042 & $01 \mathrm{~h} 38 \mathrm{~m} 52.92 \mathrm{~s}$ & $-10 \mathrm{~d} 27 \mathrm{~m} 11.4 \mathrm{~s}$ & 0.0483 & 210.0 & 11.85 & \\
\hline 2 & F04454-4838 & ESO 203-IG1 & $04 \mathrm{~h} 46 \mathrm{~m} 49.50 \mathrm{~s}$ & $-48 \mathrm{~d} 33 \mathrm{~m} 32.9 \mathrm{~s}$ & 0.0529 & 235.0 & 11.86 & \\
\hline 3 & F05081+7936 & VII Zw 31 & $05 \mathrm{~h} 16 \mathrm{~m} 46.44 \mathrm{~s}$ & $+79 \mathrm{~d} 40 \mathrm{~m} 12.6 \mathrm{~s}$ & 0.0537 & 240.0 & 11.99 & \\
\hline 4 & F05189-2524 & IRAS F05189-2524 & $05 \mathrm{~h} 21 \mathrm{~m} 01.47 \mathrm{~s}$ & $-25 \mathrm{~d} 21 \mathrm{~m} 45.4 \mathrm{~s}$ & 0.0425 & 187.0 & 12.16 & \\
\hline 5 & F06259-4708 & ESO 255-IG7 & $06 \mathrm{~h} 27 \mathrm{~m} 22.45 \mathrm{~s}$ & $-47 \mathrm{~d} 10 \mathrm{~m} 48.7 \mathrm{~s}$ & 0.0388 & 173.0 & 11.90 & \\
\hline 6 & $07251-0248$ & IRAS 07251-0248 & $07 \mathrm{~h} 27 \mathrm{~m} 37.55 \mathrm{~s}$ & $-02 \mathrm{~d} 54 \mathrm{~m} 54.1 \mathrm{~s}$ & 0.0875 & 400.0 & 12.39 & \\
\hline 7 & F08520-6850 & ESO 60-IG16 & $08 \mathrm{~h} 52 \mathrm{~m} 31.29 \mathrm{~s}$ & $-69 \mathrm{~d} 01 \mathrm{~m} 57.0 \mathrm{~s}$ & 0.0463 & 210.0 & 11.82 & \\
\hline 8 & F08572+3915 & IRAS F08572+3915 & $09 \mathrm{~h} 00 \mathrm{~m} 25.39 \mathrm{~s}$ & $+39 \mathrm{~d} 03 \mathrm{~m} 54.4 \mathrm{~s}$ & 0.0584 & 264.0 & 12.16 & \\
\hline 9 & 09022-3615 & IRAS 09022-3615 & $09 \mathrm{~h} 04 \mathrm{~m} 12.70 \mathrm{~s}$ & $-36 \mathrm{~d} 27 \mathrm{~m} 01.1 \mathrm{~s}$ & 0.0596 & 271.0 & 12.31 & \\
\hline 10 & F09111-1007 & IRAS F09111-1007 & $09 \mathrm{~h} 13 \mathrm{~m} 37.61 \mathrm{~s}$ & $-10 \mathrm{~d} 19 \mathrm{~m} 24.8 \mathrm{~s}$ & 0.0541 & 246.0 & 12.06 & \\
\hline 11 & F09126+4432 & UGC 4881 & $09 \mathrm{~h} 15 \mathrm{~m} 55.11 \mathrm{~s}$ & $+44 \mathrm{~d} 19 \mathrm{~m} 54.1 \mathrm{~s}$ & 0.0393 & 178.0 & 11.74 & Arp 55 \\
\hline 12 & F09320+6134 & UGC 5101 & $09 \mathrm{~h} 35 \mathrm{~m} 51.65 \mathrm{~s}$ & $+61 \mathrm{~d} 21 \mathrm{~m} 11.3 \mathrm{~s}$ & 0.0394 & 177.0 & 12.01 & \\
\hline 13 & F10038-3338 & ESO 374-IG 032a & $10 \mathrm{~h} 06 \mathrm{~m} 04.80 \mathrm{~s}$ & $-33 \mathrm{~d} 53 \mathrm{~m} 15.0 \mathrm{~s}$ & 0.0341 & 156.0 & 11.78 & \\
\hline 14 & $\mathrm{~F} 10173+0828$ & IRAS F10173+0828 & $10 \mathrm{~h} 20 \mathrm{~m} 00.21 \mathrm{~s}$ & $+08 \mathrm{~d} 13 \mathrm{~m} 33.8 \mathrm{~s}$ & 0.0491 & 224.0 & 11.86 & \\
\hline 15 & F10565+2448 & IRAS F10565+2448 & $10 \mathrm{~h} 59 \mathrm{~m} 18.14 \mathrm{~s}$ & $+24 \mathrm{~d} 32 \mathrm{~m} 34.3 \mathrm{~s}$ & 0.0431 & 197.0 & 12.08 & \\
\hline 16 & F11257+5850 & NGC 3690 & $11 \mathrm{~h} 28 \mathrm{~m} 32.25 \mathrm{~s}$ & $+58 \mathrm{~d} 33 \mathrm{~m} 44.0 \mathrm{~s}$ & 0.0104 & 50.7 & 11.93 & Arp 299 \\
\hline 17 & F12112+0305 & IRAS F12112+0305 & $12 \mathrm{~h} 13 \mathrm{~m} 46.00 \mathrm{~s}$ & $+02 \mathrm{~d} 48 \mathrm{~m} 38.0 \mathrm{~s}$ & 0.0733 & 340.0 & 12.36 & \\
\hline 18 & F12540+5708 & UGC 8058 & $12 \mathrm{~h} 56 \mathrm{~m} 14.23 \mathrm{~s}$ & $+56 \mathrm{~d} 52 \mathrm{~m} 25.2 \mathrm{~s}$ & 0.0422 & 192.0 & 12.57 & Mrk 231 \\
\hline 19 & $13120-5453$ & IRAS 13120-5453 & $13 \mathrm{~h} 15 \mathrm{~m} 06.35 \mathrm{~s}$ & $-55 \mathrm{~d} 09 \mathrm{~m} 22.7 \mathrm{~s}$ & 0.0308 & 144.0 & 12.32 & \\
\hline 20 & $F 13136+6223$ & VV 250a & $13 \mathrm{~h} 15 \mathrm{~m} 35.06 \mathrm{~s}$ & $+62 \mathrm{~d} 07 \mathrm{~m} 28.6 \mathrm{~s}$ & 0.0311 & 142.0 & 11.81 & Arp 238 \\
\hline 21 & $F 13182+3424$ & UGC 8387 & $13 \mathrm{~h} 20 \mathrm{~m} 35.34 \mathrm{~s}$ & $+34 \mathrm{~d} 08 \mathrm{~m} 22.2 \mathrm{~s}$ & 0.0233 & 110.0 & 11.73 & IC 883, Arp 193 \\
\hline 22 & F13428+5608 & UGC 8696 & $13 \mathrm{~h} 44 \mathrm{~m} 42.11 \mathrm{~s}$ & $+55 \mathrm{~d} 53 \mathrm{~m} 12.7 \mathrm{~s}$ & 0.0378 & 173.0 & 12.21 & Mrk 273 \\
\hline 23 & F14348-1447 & IRAS F14348-1447 & $14 \mathrm{~h} 37 \mathrm{~m} 38.37 \mathrm{~s}$ & $-15 \mathrm{~d} 00 \mathrm{~m} 22.8 \mathrm{~s}$ & 0.0827 & 387.0 & 12.39 & \\
\hline 24 & F14378-3651 & IRAS F14378-3651 & $14 \mathrm{~h} 40 \mathrm{~m} 59.01 \mathrm{~s}$ & $-37 \mathrm{~d} 04 \mathrm{~m} 32.0 \mathrm{~s}$ & 0.0676 & 315.0 & 12.23 & \\
\hline 25 & F14547+2449 & VV 340a & $14 \mathrm{~h} 57 \mathrm{~m} 00.68 \mathrm{~s}$ & $+24 \mathrm{~d} 37 \mathrm{~m} 02.7 \mathrm{~s}$ & 0.0337 & 157.0 & 11.74 & Arp 302 \\
\hline 26 & $\mathrm{~F} 15163+4255$ & VV 705 & $15 \mathrm{~h} 18 \mathrm{~m} 06.28 \mathrm{~s}$ & $+42 \mathrm{~d} 44 \mathrm{~m} 41.2 \mathrm{~s}$ & 0.0402 & 183.0 & 11.92 & I Zw 107 \\
\hline 27 & $F 15250+3608$ & IRAS F15250+3608 & $15 \mathrm{~h} 26 \mathrm{~m} 59.40 \mathrm{~s}$ & $+35 \mathrm{~d} 58 \mathrm{~m} 37.5 \mathrm{~s}$ & 0.0552 & 254.0 & 12.00 & \\
\hline 28 & $F 15327+2340$ & UGC 9913 & $15 \mathrm{~h} 34 \mathrm{~m} 57.12 \mathrm{~s}$ & $+23 \mathrm{~d} 30 \mathrm{~m} 11.5 \mathrm{~s}$ & 0.0182 & 87.9 & 12.28 & Arp 220 \\
\hline 29 & F16330-6820 & ESO 69-IG6 & $16 \mathrm{~h} 38 \mathrm{~m} 12.65 \mathrm{~s}$ & $-68 d 26 m 42.6 s$ & 0.0464 & 212.0 & 11.98 & \\
\hline 30 & $\mathrm{~F} 16504+0228$ & NGC 6240 & $16 \mathrm{~h} 52 \mathrm{~m} 58.89 \mathrm{~s}$ & $+02 \mathrm{~d} 24 \mathrm{~m} 03.4 \mathrm{~s}$ & 0.0245 & 116.0 & 11.93 & \\
\hline 31 & $\mathrm{~F} 17132+5313$ & IRAS F17132+5313 & $17 \mathrm{~h} 14 \mathrm{~m} 20.00 \mathrm{~s}$ & $+53 \mathrm{~d} 10 \mathrm{~m} 30.0 \mathrm{~s}$ & 0.0509 & 232.0 & 11.96 & \\
\hline 32 & F17207-0014 & IRAS F17207-0014 & $17 \mathrm{~h} 23 \mathrm{~m} 21.96 \mathrm{~s}$ & $-00 \mathrm{~d} 17 \mathrm{~m} 00.9 \mathrm{~s}$ & 0.0428 & 198.0 & 12.46 & \\
\hline 33 & F18293-3413 & IRAS F18293-3413 & $18 \mathrm{~h} 32 \mathrm{~m} 41.13 \mathrm{~s}$ & $-34 \mathrm{~d} 11 \mathrm{~m} 27.5 \mathrm{~s}$ & 0.0182 & 86.0 & 11.88 & \\
\hline 34 & F19115-2124 & ESO 593-IG8 & $19 \mathrm{~h} 14 \mathrm{~m} 30.90 \mathrm{~s}$ & $-21 \mathrm{~d} 19 \mathrm{~m} 07.0 \mathrm{~s}$ & 0.0487 & 222.0 & 11.93 & \\
\hline 35 & F19297-0406 & IRAS F19297-0406 & $19 \mathrm{~h} 32 \mathrm{~m} 21.25 \mathrm{~s}$ & $-03 \mathrm{~d} 59 \mathrm{~m} 56.3 \mathrm{~s}$ & 0.0857 & 395.0 & 12.45 & \\
\hline 36 & $19542+1110$ & IRAS 19542+1110 & $19 \mathrm{~h} 56 \mathrm{~m} 35.44 \mathrm{~s}$ & $+11 \mathrm{~d} 19 \mathrm{~m} 02.6 \mathrm{~s}$ & 0.0650 & 295.0 & 12.12 & \\
\hline 37 & F20550+1655 & CGCG 448-020 & $20 \mathrm{~h} 57 \mathrm{~m} 23.90 \mathrm{~s}$ & $+17 \mathrm{~d} 07 \mathrm{~m} 39.0 \mathrm{~s}$ & 0.0361 & 161.0 & 11.94 & II Zw 96 \\
\hline 38 & F20551-4250 & ESO 286-IG19 & $20 \mathrm{~h} 58 \mathrm{~m} 26.79 \mathrm{~s}$ & $-42 \mathrm{~d} 39 \mathrm{~m} 00.3 \mathrm{~s}$ & 0.0430 & 193.0 & 12.06 & \\
\hline 39 & $21101+5810$ & IRAS $21101+5810$ & $21 \mathrm{~h} 11 \mathrm{~m} 30.40 \mathrm{~s}$ & $+58 \mathrm{~d} 23 \mathrm{~m} 03.2 \mathrm{~s}$ & 0.0390 & 174.0 & 11.81 & \\
\hline 40 & F22467-4906 & ESO 239-IG2 & $22 \mathrm{~h} 49 \mathrm{~m} 39.87 \mathrm{~s}$ & $-48 \mathrm{~d} 50 \mathrm{~m} 58.1 \mathrm{~s}$ & 0.0430 & 191.0 & 11.84 & \\
\hline 41 & F22491-1808 & IRAS F22491-1808 & $22 \mathrm{~h} 51 \mathrm{~m} 49.26 \mathrm{~s}$ & $-17 \mathrm{~d} 52 \mathrm{~m} 23.5 \mathrm{~s}$ & 0.0778 & 351.0 & 12.20 & \\
\hline 42 & F23128-5919 & ESO 148-IG2 & $23 \mathrm{~h} 15 \mathrm{~m} 46.78 \mathrm{~s}$ & $-59 \mathrm{~d} 03 \mathrm{~m} 15.6 \mathrm{~s}$ & 0.0446 & 199.0 & 12.06 & \\
\hline 43 & F23180-6929 & ESO 77-IG14 & $23 \mathrm{~h} 21 \mathrm{~m} 04.53 \mathrm{~s}$ & $-69 \mathrm{~d} 12 \mathrm{~m} 54.2 \mathrm{~s}$ & 0.0416 & 186.0 & 11.76 & \\
\hline 44 & $\mathrm{~F} 23365+3604$ & IRAS F23365+3604 & $23 \mathrm{~h} 39 \mathrm{~m} 01.27 \mathrm{~s}$ & $+36 \mathrm{~d} 21 \mathrm{~m} 08.7 \mathrm{~s}$ & 0.0645 & 287.0 & 12.20 & \\
\hline
\end{tabular}

Notes. ${ }^{(a)}$ When the IRAS Revised Bright Galaxy Sample (RBGS, Sanders et al. 2003) was compiled, IRAS F10038-3338 was mistakenly crossidentified with the optical source IC 2545. The proper optical counterpart is ESO 374-IG 032. (See the Essential Notes in NED.) Column (1): through number of the object, which are also used in other tables and Fig. 2. Column (2): original IRAS source, where an "F" prefix indicates the Faint Source Catalog and no prefix indicates the Point Source Catalog. Column (3): optical cross-identification, where available from NED. Column (4): the best available source right ascension (J2000) in NED as of October 2008. Column (5): the best available source declination (J2000) in NED as of October 2008. Column (6): the best available heliocentric redshift, in NED as of October 2008. Column (7): the luminosity distance in megaparsecs derived by correrecting the heliocentric velocity for the 3-attractor flow model of Mould et al. (2000) and adopting cosmological parameters $H_{0}=70 \mathrm{~km} \mathrm{~s}^{-1} \mathrm{Mpc}^{-2}, \Omega_{\mathrm{V}}=0.72$, and $\Omega_{\mathrm{M}}=0.28$ based on the 5-year WMAP results (Hinshaw et al. 2009), as provided by NED. Column (8): the total infrared luminosity in $\log _{10}$ Solar units computed using the flux densities reported in the RBGS and the lumiosity distances in Col. (7) using the formulae $L_{\text {ir }} / L_{\odot}=4 \pi\left(D_{\mathrm{L}[\mathrm{m}]}\right)^{2}\left(F_{\text {ir }}\left[\mathrm{W} \mathrm{m}^{-2}\right]\right) / 3.826 \times 10^{26}\left[\mathrm{~W} \mathrm{~m}^{-2}\right]$, where $F_{\text {ir }}=1.8 \times 10^{-14}\left\{13.48 f_{12 \mu \mathrm{m}}[\mathrm{Jy}]+5.16 f_{25} \mu \mathrm{m}[\mathrm{Jy}]+\right.$ $\left.2.58 f_{60 \mu \mathrm{m}}[\mathrm{Jy}]+f_{100 \mu \mathrm{m}}[\mathrm{Jy}]\right\}\left[\mathrm{W} \mathrm{m}^{-2}\right]$ (Sanders \& Mirabel 1996). Column (9): other conventionally used object name.

\subsection{Images}

Figure 2 shows the X-ray brightness contours overlaid on the HST-ACS F814W ( $I$-band) image (Evans et al., in prep.) for all 44 targets. The contours are made from a $0.4-7 \mathrm{keV}$ image, smoothed using a circular Gaussian filter with typical dispersion of 1 arcsec. Note that the deep image of Mrk 231 reaches a significantly lower brightness level, and thus the contour levels are drawn down to a level which is a factor of 10 lower than for the other objects.

The appearance of the X-ray images often differs dramatically between the soft and hard bands. In addition to the full band $(0.4-7 \mathrm{keV})$ image in unsmoothed $\left(0.5^{\prime \prime} \times 0.5^{\prime \prime}\right.$ pixel size $)$ and smoothed forms, images of the same region of the sky in the 
Table 2. Chandra observation log.

\begin{tabular}{|c|c|c|c|c|c|c|c|}
\hline No. & Galaxy & Obs ID & Date & Mode & $\begin{array}{l}\text { Exp. Time } \\
\text { (ks) }\end{array}$ & $\begin{array}{c}0.4-7 \mathrm{keV}^{a} \\
(\mathrm{cts})\end{array}$ & $\begin{array}{c}N_{\mathrm{H}, \mathrm{Gal}}{ }^{b} \\
\left(10^{20} \mathrm{~cm}^{-2}\right)\end{array}$ \\
\hline \multicolumn{8}{|c|}{ Cycle 8 targets } \\
\hline 1 & F01364-1042 & 7801 & 2007 Sep. 10 & VFAINT & 14.57 & $46.0 \pm 7.3$ & 2.0 \\
\hline 2 & ESO 203-IG1 & 7802 & 2008 Jan. 17 & VFAINT & 14.85 & $0(<3)$ & 1.4 \\
\hline 3 & VII Zw 31 & 7887 & 2007 May 27 & VFAINT & 14.98 & $173.8 \pm 13.3$ & 7.4 \\
\hline 5 & ESO 255-IG7 & 7803 & 2007 May 27 & VFAINT & 14.57 & $341.8 \pm 18.8$ & 3.8 \\
\hline 6 & 07251-0248 & 7804 & 2006 Dec. 01 & VFAINT & 15.43 & $12.7 \pm 3.6$ & 14.6 \\
\hline 7 & ESO 60-IG16 & 7888 & 2007 May 31 & VFAINT & 14.68 & $122.6 \pm 11.1$ & 5.2 \\
\hline 9 & 09022-3615 & 7805 & 2007 Sep. 04 & VFAINT & 14.85 & $265.3 \pm 16.5$ & 26.6 \\
\hline 10 & F09111-1007 & 7806 & 2007 Mar. 20 & VFAINT & 14.63 & $118.7 \pm 11.1$ & 4.6 \\
\hline 13 & ESO 374-IG32 & 7807 & 2007 Mar. 07 & VFAINT & 14.36 & $75.0 \pm 9.1$ & 8.8 \\
\hline 14 & F10173+0828 & 7808 & 2008 Jan. 18 & VFAINT & 14.98 & $9.8 \pm 3.2$ & 2.3 \\
\hline 19 & $13120-5453$ & 7809 & 2006 Dec. 01 & VFAINT & 14.67 & $300.7 \pm 17.4$ & 21.3 \\
\hline 20 & VV 250 & 7810 & 2007 Aug. 22 & VFAINT & 14.85 & $391.9 \pm 20.1$ & 2.0 \\
\hline 21 & UGC 8387 & 7811 & 2007 Feb. 19 & VFAINT & 14.07 & $251.4 \pm 16.0$ & 1.0 \\
\hline 24 & F14378-3651 & 7889 & 2007 Jun. 25 & VFAINT & 13.86 & $45.3 \pm 6.8$ & 6.3 \\
\hline 25 & VV 340 & 7812 & 2006 Dec. 17 & VFAINT & 14.86 & $331.4 \pm 20.4$ & 3.3 \\
\hline 29 & ESO 69-IG6 & 7813 & 2007 Jun. 21 & VFAINT & 14.54 & $330.1 \pm 18.3$ & 9.1 \\
\hline 31 & F17132+5313 & 7814 & 2007 Apr. 03 & VFAINT & 14.85 & $90.8 \pm 10.0$ & 1.9 \\
\hline 33 & F18293-3413 & 7815 & 2007 Feb. 25 & VFAINT & 14.04 & $444.6 \pm 21.5$ & 9.7 \\
\hline 34 & ESO 593-IG8 & 7816 & 2007 Jun. 09 & VFAINT & 14.97 & $158.1 \pm 13.7$ & 8.1 \\
\hline 35 & F19297-0406 & 7980 & 2007 Jun. 18 & VFAINT & 16.42 & $85.8 \pm 9.7$ & 15.1 \\
\hline 36 & $19542+1110$ & 7817 & 2007 Sep. 10 & VFAINT & 14.98 & $324.3 \pm 18.0$ & 14.0 \\
\hline 37 & CGCG 448-020 & 7818 & 2007 Sep. 10 & VFAINT & 14.56 & $301.3 \pm 18.2$ & 6.9 \\
\hline 39 & 21101+5810 & 7819 & 2007 Jul. 01 & VFAINT & 14.85 & $21.7 \pm 4.8$ & 37.2 \\
\hline 40 & ESO 239-IG2 & 7820 & 2007 Sep. 10 & VFATIN & 14.57 & $151.5 \pm 13.1$ & 0.9 \\
\hline 41 & F22491-1808 & 7821 & 2007 Jul. 13 & VFAINT & 14.97 & $50.9 \pm 7.3$ & 2.3 \\
\hline 43 & ESO 77-IG14 & 7822 & 2008 Jan. 26 & VFAINT & 14.98 & $84.1 \pm 9.3$ & 3.3 \\
\hline \multicolumn{8}{|c|}{ Archival data } \\
\hline 4 & F05189-2524 & 3432 & 2002 Jan. 03 & FAINT & 14.86 & $2016.9 \pm 45.0$ & 1.7 \\
\hline 8 & F08572+3915 & 6862 & 2006 Jan. 26 & FAINT & 14.94 & $9.7 \pm 3.2$ & 2.0 \\
\hline 11 & UGC 4881 & 6857 & 2006 Jan. 12 & FAINT & 14.77 & $69.4 \pm 8.4$ & 1.4 \\
\hline 12 & UGC 5101 & 2033 & 2001 May 28 & FAINT & 49.32 & $482.9 \pm 22.3$ & 3.0 \\
\hline 15 & F10565+2448 & 4552 & 2003 Oct. 23 & FAINT & 28.87 & $335.2 \pm 18.8$ & 1.1 \\
\hline 16 & NGC 3690 & 6227 & 2005 Feb. 14 & FAINT & 10.19 & $2526.0 \pm 52.1$ & 0.9 \\
\hline 17 & $\mathrm{~F} 12112+0305$ & 4110 & 2003 Apr. 15 & VFAINT & 9.87 & $42.6 \pm 6.6$ & 1.8 \\
\hline 18 & UGC 8058 & $1031^{c}$ & 2000 Oct 19 & FAINT & 39.25 & $2312.5 \pm 66.2$ & 1.0 \\
\hline 18 & UGC 8058 & 4028 & 2003 Feb. 03 & VFAINT & 39.68 & $2205.6 \pm 59.7$ & 1.0 \\
\hline 18 & UGC 8058 & 4029 & 2003 Feb. 11 & VFAINT & 38.63 & $2070.5 \pm 57.3$ & 1.0 \\
\hline 18 & UGC 8058 & 4030 & 2003 Feb. 20 & VFAINT & 36.01 & $1876.6 \pm 52.0$ & 1.0 \\
\hline 22 & UGC 8696 & 809 & 2000 Apr. 19 & VFAINT & 44.19 & $2054.0 \pm 45.8$ & 0.9 \\
\hline 23 & F14348-1447 & 6861 & 2006 Mar. 12 & FAINT & 14.72 & $75.9 \pm 8.8$ & 7.5 \\
\hline 26 & VV 705 & 6858 & 2006 Sep. 11 & FAINT & 14.47 & $157.8 \pm 12.6$ & 1.8 \\
\hline 27 & F15250+3608 & 4112 & 2003 Aug. 27 & VFAINT & 9.84 & $26.6 \pm 5.3$ & 1.5 \\
\hline 28 & UGC 9913 & 869 & 2000 Jun. 24 & FAINT & 56.49 & $1555.1 \pm 47.1$ & 3.9 \\
\hline 30 & NGC 6240 & 1590 & 2001 Jul. 29 & FAINT & 36.69 & $10010.7 \pm 103.5$ & 4.9 \\
\hline 32 & F17207-0014 & 2035 & 2001 Oct. 24 & FAINT & 48.53 & $476.6 \pm 23.1$ & 9.7 \\
\hline 38 & ESO 286-IG19 & 2036 & 2001 Oct. 31 & FAINT & 44.87 & $767.8 \pm 28.3$ & 3.3 \\
\hline 42 & ESO 148-IG2 & 2037 & 2001 Sep. 30 & FAINT & 49.31 & $1052.2 \pm 34.9$ & 1.6 \\
\hline 44 & F23365+3604 & 4115 & 2003 Feb. 03 & VFAINT & 10.10 & $28.8 \pm 5.4$ & 9.6 \\
\hline
\end{tabular}

Notes. The 26 Cycle 8 Chandra observations (PI: D. Sanders) are listed first, followed by archival data for the additional 18 objects with $\log \left(L_{\mathrm{ir}} / L_{\odot}\right)>11.73$. All observations were obtained in imaging mode with the ACIS-S, and the targets were placed at the nominal pointing position on the detector. ${ }^{(a)}$ The source counts are corrected for background and measured in the $0.4-7 \mathrm{keV}$ band. The counts from separate components in a single objects are summed together. ${ }^{(b)}$ The Galactic absorption column density is taken from the LAB HI map by Kalberla et al. (2005). ${ }^{(c)}$ This observation was used to make the radial surface-brightness profile.

soft (0.5-2 keV) and hard (2-7 keV) bands are shown. The same smoothing has been applied to the $0.5-2 \mathrm{keV}$ and $2-7 \mathrm{keV}$ images. Linear scaling is normally used for presentation, but for a few sources with strongly peaked emission, a logarithmic scale was used to show faint extended features. The scale bar represents an angular scale of 5 arcsec. The soft $(0.5-2 \mathrm{keV})$ and hard (2-7 keV) band radial profiles, described in the following section, are also shown. An example for these figures is shown in Fig. 3 for UGC 8387. Similar style multi-panel figures were made for all $44 \mathrm{C}$-GOALS targets and these can be found in the Appendix.

As seen clearly in UGC 8387, soft X-ray nebulae extending along the minor axis of the galaxy, or in the direction displaced from the optical light distribution, are often observed. These extended SX nebulae are likely associated with a galactic-scale outflow from the nuclear region, driven by either a starburst or 
Table 3. X-ray spectral properties for the C-GOALS sample.

\begin{tabular}{|c|c|c|c|c|c|c|c|c|c|c|}
\hline No. & Galaxy & $\begin{array}{l}S X \\
\text { (1) }\end{array}$ & $\begin{array}{l}H X \\
(2)\end{array}$ & $\begin{array}{c}H R \\
(3)\end{array}$ & $\begin{array}{l}F_{\mathrm{SX}} \\
(4)\end{array}$ & $\begin{array}{c}F_{\mathrm{HX}} \\
(5)\end{array}$ & $\begin{array}{c}L_{\mathrm{SX}} \\
(6)\end{array}$ & $\begin{array}{l}L_{\mathrm{HX}} \\
(7)\end{array}$ & $\begin{array}{c}S X / I R \\
(8)\end{array}$ & $\begin{array}{c}H X / I R \\
(9)\end{array}$ \\
\hline \multicolumn{11}{|c|}{ Cycle 8 Data } \\
\hline 1 & F01364-1042 & $2.14 \pm 0.41$ & $0.86 \pm 0.30$ & $-0.43 \pm 0.18$ & $4.5 e-15$ & $2.0 \mathrm{e}-14$ & $2.4 \mathrm{e} 40$ & $1.5 \mathrm{e} 41$ & -5.05 & -4.26 \\
\hline 2 & ESO 203-IG1 & $0.00 \pm 0.20$ & $0.00 \pm 0.20$ & - & $<1.5 \mathrm{e}-15$ & $<3.0 \mathrm{e}-15$ & $<5.6 \mathrm{e} 39$ & $<6.3 \mathrm{e} 40$ & $<-5.69$ & $<-5.14$ \\
\hline 3 & VII Zw 31 & $9.49 \pm 0.80$ & $2.01 \pm 0.38$ & $-0.65 \pm 0.09$ & $3.0 \mathrm{e}-14$ & $3.5 \mathrm{e}-14$ & $2.0 \mathrm{e} 41$ & $2.7 \mathrm{e} 41$ & -4.27 & -4.14 \\
\hline 5 & ESO 255-IG7 N & $12.40 \pm 0.93$ & $2.77 \pm 0.46$ & $-0.64 \pm 0.08$ & $3.5 \mathrm{e}-14$ & $2.9 \mathrm{e}-14$ & $1.2 \mathrm{e} 41$ & $1.6 \mathrm{e} 41$ & -4.41 & -4.28 \\
\hline 5 & ESO 255-IG7 C & $4.77 \pm 0.57$ & $1.35 \pm 0.32$ & $-0.56 \pm 0.12$ & $1.5 \mathrm{e}-14$ & $2.1 \mathrm{e}-14$ & $5.3 \mathrm{e} 40$ & $1.2 \mathrm{e} 41$ & -4.76 & -4.41 \\
\hline 5 & ESO 255-IG7 S & $1.53 \pm 0.33$ & $0.63 \pm 0.23$ & $-0.42 \pm 0.20$ & $4.5 \mathrm{e}-15$ & $6.8 \mathrm{e}-15$ & $1.5 \mathrm{e} 40$ & $3.8 \mathrm{e} 40$ & -5.31 & -4.90 \\
\hline 6 & 07251-0248 & $0.77 \pm 0.22$ & $0.05 \pm 0.06$ & $-0.87 \pm 0.38$ & $3.3 \mathrm{e}-15$ & $<3 e-15$ & $6.2 \mathrm{e} 40$ & $<5.8 \mathrm{e} 40$ & -5.18 & $<-5.21$ \\
\hline 7 & ESO 60-IG16 & $3.24 \pm 0.47$ & $5.09 \pm 0.59$ & $+0.22 \pm 0.09$ & $8.1 \mathrm{e}-15$ & $1.0 \mathrm{e}-13$ & $5.5 \mathrm{e} 40$ & $7.3 \mathrm{e} 41$ & -4.66 & -3.54 \\
\hline 9 & 09022-3615 & $10.45 \pm 0.84$ & $7.38 \pm 0.72$ & $-0.17 \pm 0.06$ & $2.9 \mathrm{e}-14$ & $1.4 \mathrm{e}-13$ & $4.4 \mathrm{e} 41$ & $2.0 \mathrm{e} 42$ & -4.25 & -3.59 \\
\hline 10 & F09111-1007E & $5.27 \pm 0.60$ & $0.97 \pm 0.27$ & $-0.69 \pm 0.13$ & $1.6 \mathrm{e}-14$ & $1.2 \mathrm{e}-14$ & $1.2 \mathrm{e} 41$ & $9.8 \mathrm{e} 40$ & -4.57 & -4.65 \\
\hline 10 & F09111-1007W & $1.20 \pm 0.29$ & $0.55 \pm 0.21$ & $-0.37 \pm 0.22$ & $3.6 \mathrm{e}-15$ & $4.5 e-15$ & $2.1 \mathrm{e} 40$ & $3.8 \mathrm{e} 40$ & -5.32 & -5.06 \\
\hline 13 & ESO 374-IG32 & $4.02 \pm 0.55$ & $1.22 \pm 0.32$ & $-0.62 \pm 0.13$ & $1.3 \mathrm{e}-14$ & $1.7 \mathrm{e}-14$ & $3.8 \mathrm{e} 40$ & $6.3 \mathrm{e} 40$ & -4.78 & -4.56 \\
\hline 14 & F10173+0828 & $0.59 \pm 0.21$ & $0.06 \pm 0.06$ & $-0.82 \pm 0.42$ & $2.1 \mathrm{e}-15$ & $1.0 \mathrm{e}-15$ & $1.1 \mathrm{e} 40$ & $6.6 \mathrm{e} 39$ & -5.40 & -5.62 \\
\hline 19 & $13120-5453$ & $11.63 \pm 0.89$ & $9.00 \pm 0.79$ & $-0.13 \pm 0.06$ & $3.7 \mathrm{e}-14$ & $1.4 \mathrm{e}-13$ & $1.1 \mathrm{e} 41$ & $4.5 \mathrm{e} 41$ & -4.86 & -4.25 \\
\hline 20 & VV $250 \mathrm{E}$ & $16.90 \pm 1.08$ & $6.94 \pm 1.75$ & $-0.42 \pm 0.06$ & $5.3 \mathrm{e}-14$ & $9.5 e-14$ & $1.2 \mathrm{e} 41$ & $3.1 \mathrm{e} 41$ & -4.32 & -3.90 \\
\hline 20 & VV $250 \mathrm{~W}$ & $1.78 \pm 0.36$ & $0.54 \pm 0.22$ & $-0.53 \pm 0.20$ & $6.0 \mathrm{e}-15$ & $1.0 \mathrm{e}-15$ & $1.3 \mathrm{e} 40$ & $2.5 \mathrm{e} 39$ & -5.28 & -6.00 \\
\hline 21 & UGC 8387 & $13.77 \pm 0.99$ & $3.98 \pm 0.54$ & $-0.55 \pm 0.07$ & $4.4 \mathrm{e}-14$ & $4.3 e-14$ & $5.4 \mathrm{e} 40$ & $6.4 \mathrm{e} 40$ & -4.58 & -4.51 \\
\hline 24 & F14378-3651 & $1.92 \pm 0.38$ & $1.34 \pm 0.31$ & $-0.18 \pm 0.15$ & $6.7 e-15$ & $2.1 \mathrm{e}-14$ & $8.0 \mathrm{e} 40$ & $3.4 \mathrm{e} 41$ & -4.91 & -4.28 \\
\hline 25 & VV $340 \mathrm{~N}$ & $16.58 \pm 1.08$ & $2.55 \pm 0.55$ & $-0.74 \pm 0.08$ & $4.8 \mathrm{e}-14$ & $7.3 e-14$ & $1.3 \mathrm{e} 41$ & $2.9 \mathrm{e} 41$ & -4.21 & -3.86 \\
\hline 25 & VV $340 \mathrm{~S}$ & $2.35 \pm 0.41$ & $0.92 \pm 0.33$ & $-0.44 \pm 0.18$ & $6.6 \mathrm{e}-15$ & $3.6 e-15$ & $1.7 \mathrm{e} 40$ & $1.2 \mathrm{e} 40$ & -5.09 & -5.25 \\
\hline 29 & ESO 69-IG6 (N) & $8.88 \pm 0.79$ & $1.78 \pm 0.37$ & $-0.67 \pm 0.10$ & $2.8 \mathrm{e}-14$ & $1.8 \mathrm{e}-14$ & $1.4 \mathrm{e} 41$ & $1.0 \mathrm{e} 41$ & -4.42 & -4.56 \\
\hline 29 & ESO 69-IG6 (S) & $2.19 \pm 0.39$ & $9.97 \pm 0.83$ & $+0.64 \pm 0.09$ & $8.1 \mathrm{e}-15$ & $1.7 \mathrm{e}-13$ & $3.5 \mathrm{e} 40$ & $1.2 \mathrm{e} 42$ & -5.02 & -3.49 \\
\hline 31 & $\mathrm{~F} 17132+5313$ & $5.13 \pm 0.60$ & $0.76 \pm 0.33$ & $-0.74 \pm 0.14$ & $1.3 \mathrm{e}-14$ & $1.1 \mathrm{e}-14$ & $8.3 \mathrm{e} 40$ & $8.6 \mathrm{e} 40$ & -4.63 & -4.61 \\
\hline 33 & F18293-3413 & $22.92 \pm 1.29$ & $8.66 \pm 0.82$ & $-0.45 \pm 0.05$ & $6.9 \mathrm{e}-14$ & $1.3 \mathrm{e}-13$ & $5.7 \mathrm{e} 40$ & $9.4 \mathrm{e} 40$ & -4.71 & -4.49 \\
\hline 34 & ESO 593-IG8 & $9.01 \pm 0.81$ & $1.65 \pm 0.44$ & $-0.69 \pm 0.11$ & $2.1 \mathrm{e}-14$ & $2.5 \mathrm{e}-14$ & $1.2 \mathrm{e} 41$ & $1.9 \mathrm{e} 41$ & -4.44 & -4.24 \\
\hline 35 & F19297-0406 & $3.85 \pm 0.49$ & $1.21 \pm 0.30$ & $-0.52 \pm 0.13$ & $1.2 \mathrm{e}-14$ & $8.2 \mathrm{e}-15$ & $3.0 \mathrm{e} 41$ & $1.8 \mathrm{e} 41$ & -4.56 & -4.78 \\
\hline 36 & $19542+1110$ & $4.80 \pm 0.57$ & $17.12 \pm 1.07$ & $+0.56 \pm 0.06$ & $1.8 \mathrm{e}-14$ & $2.9 \mathrm{e}-13$ & $2.2 \mathrm{e} 41$ & $4.1 \mathrm{e} 42$ & -4.36 & -3.09 \\
\hline 37 & CGCG 448-020 & $15.91 \pm 1.07$ & $4.41 \pm 0.64$ & $-0.57 \pm 0.07$ & $4.9 \mathrm{e}-14$ & $6.7 \mathrm{e}-14$ & $1.4 \mathrm{e} 41$ & $2.8 \mathrm{e} 41$ & -4.38 & -4.08 \\
\hline 39 & $21101+5810$ & $1.17 \pm 0.29$ & $0.33 \pm 0.17$ & $-0.56 \pm 0.25$ & $3.5 \mathrm{e}-15$ & $4.0 \mathrm{e}-15$ & $1.9 \mathrm{e} 40$ & $2.0 \mathrm{e} 40$ & -5.12 & -5.09 \\
\hline 40 & ESO 239-IG2 & $9.14 \pm 0.83$ & $1.31 \pm 0.36$ & $-0.75 \pm 0.11$ & $3.0 \mathrm{e}-14$ & $1.9 \mathrm{e}-14$ & $1.4 \mathrm{e} 41$ & $9.7 \mathrm{e} 40$ & -4.28 & -4.44 \\
\hline 41 & F22491-1808 & $3.19 \pm 0.47$ & $0.14 \pm 0.14$ & $-0.91 \pm 0.20$ & $8.3 e-15$ & $3.0 \mathrm{e}-15$ & $1.3 \mathrm{e} 41$ & $0.6 \mathrm{e} 41$ & -4.67 & -5.01 \\
\hline 43 & ESO 77-IG14 N & $2.65 \pm 0.42$ & $0.94 \pm 0.26$ & $-0.47 \pm 0.15$ & $7.8 \mathrm{e}-15$ & $1.7 \mathrm{e}-14$ & $9.6 \mathrm{e} 40$ & $6.8 \mathrm{e} 40$ & -4.36 & -4.51 \\
\hline 43 & ESO 77-IG14 S & $1.85 \pm 0.35$ & $0.22 \pm 0.13$ & $-0.78 \pm 0.23$ & $6.0 \mathrm{e}-15$ & $6.1 \mathrm{e}-15$ & $2.4 \mathrm{e} 40$ & $2.5 \mathrm{e} 40$ & -4.96 & -4.95 \\
\hline \multicolumn{11}{|c|}{ Archival Data } \\
\hline 4 & F05189-2524 & $27.15 \pm 1.35$ & $107.00 \pm 2.69$ & $+0.60 \pm 0.03$ & $8.2 \mathrm{e}-14$ & $2.0 \mathrm{e}-12$ & $3.4 \mathrm{e} 41$ & $1.3 \mathrm{e} 43$ & -4.21 & -2.63 \\
\hline 8 & F08572+3915 & $0.12 \pm 0.09$ & $0.45 \pm 0.18$ & $+0.57 \pm 0.41$ & $9.0 \mathrm{e}-15$ & $2.5 \mathrm{e}-14$ & $8.0 \mathrm{e} 40$ & $2.0 \mathrm{e} 41$ & -4.83 & -4.44 \\
\hline 11 & UGC 4881 E & $2.09 \pm 0.38$ & $0.32 \pm 0.15$ & $-0.73 \pm 0.21$ & $6.7 \mathrm{e}-15$ & $7.8 \mathrm{e}-15$ & $2.5 \mathrm{e} 40$ & $4.1 \mathrm{e} 40$ & -4.82 & -4.61 \\
\hline 11 & UGC $4881 \mathrm{~W}$ & $2.13 \pm 0.38$ & $0.13 \pm 0.12$ & $-0.88 \pm 0.24$ & $7.1 \mathrm{e}-15$ & $3.0 \mathrm{e}-15$ & $2.7 \mathrm{e} 40$ & $1.3 \mathrm{e} 40$ & -4.19 & -4.51 \\
\hline 12 & UGC 5101 & $6.34 \pm 0.36$ & $3.49 \pm 0.27$ & $-0.29 \pm 0.05$ & $1.9 \mathrm{e}-14$ & $9.1 \mathrm{e}-14$ & $7.0 \mathrm{e} 40$ & $4.7 \mathrm{e} 41$ & -4.74 & -3.92 \\
\hline 15 & $\mathrm{~F} 10565+2448$ & $9.37 \pm 0.58$ & $2.21 \pm 0.30$ & $-0.62 \pm 0.07$ & $2.8 \mathrm{e}-14$ & $2.8 \mathrm{e}-14$ & $1.2 \mathrm{e} 41$ & $1.6 \mathrm{e} 41$ & -4.58 & -4.46 \\
\hline 16 & NGC $3690 \mathrm{~T}$ & $204.84 \pm 4.64$ & $41.40 \pm 2.17$ & $-0.66 \pm 0.03$ & $6.2 \mathrm{e}-13$ & $6.8 \mathrm{e}-13$ & $1.9 \mathrm{e} 41$ & $2.6 \mathrm{e} 41$ & -4.23 & -4.10 \\
\hline 16 & NGC 3690 E & $54.46 \pm 2.33$ & $15.43 \pm 1.24$ & $-0.56 \pm 0.04$ & $1.5 \mathrm{e}-13$ & $2.6 e-13$ & $7.7 \mathrm{e} 40$ & $9.6 \mathrm{e} 40$ & -4.49 & -4.40 \\
\hline 16 & NGC $3690 \mathrm{~W}$ & $58.71 \pm 2.42$ & $15.04 \pm 1.23$ & $-0.59 \pm 0.04$ & $1.6 \mathrm{e}-13$ & $2.6 \mathrm{e}-13$ & $8.0 \mathrm{e} 40$ & $9.6 \mathrm{e} 40$ & -4.02 & -3.94 \\
\hline 17 & $\mathrm{~F} 12112+0305$ & $3.22 \pm 0.57$ & $1.09 \pm 0.34$ & $-0.49 \pm 0.17$ & $9.8 \mathrm{e}-15$ & $1.9 \mathrm{e}-14$ & $1.3 \mathrm{e} 41$ & $4.0 \mathrm{e} 41$ & -4.83 & -4.34 \\
\hline 18 & UGC 8058 & $34.23 \pm 1.04$ & $19.65 \pm 0.74$ & $-0.27 \pm 0.03$ & $9.5 \mathrm{e}-14$ & $4.0 \mathrm{e}-13$ & $3.9 \mathrm{e} 41$ & $3.0 \mathrm{e} 42$ & -4.56 & -3.67 \\
\hline 22 & UGC 8696 core & $29.02 \pm 0.82$ & $16.20 \pm 0.62$ & $-0.28 \pm 0.02$ & $7.4 \mathrm{e}-14$ & $4.2 \mathrm{e}-13$ & $2.5 \mathrm{e} 41$ & $2.5 \mathrm{e} 42$ & -4.39 & -3.39 \\
\hline 22 & UGC 8696 tail & $7.09 \pm 0.50$ & $0.00 \pm 0.31$ & $-1.00 \pm 0.12$ & $1.7 \mathrm{e}-14$ & $2.5 \mathrm{e}-15$ & $5.6 \mathrm{e} 40$ & $1.1 \mathrm{e} 40$ & - & - \\
\hline 23 & F14348-1447 T & $3.80 \pm 0.51$ & $1.32 \pm 0.31$ & $-0.48 \pm 0.13$ & $1.3 \mathrm{e}-14$ & $2.4 \mathrm{e}-14$ & $2.9 \mathrm{e} 41$ & $7.5 \mathrm{e} 41$ & -4.51 & -4.09 \\
\hline 23 & F14348-1447 S & $1.15 \pm 0.28$ & $0.80 \pm 0.24$ & $-0.18 \pm 0.19$ & $4.1 \mathrm{e}-15$ & $1.4 \mathrm{e}-14$ & $9.0 \mathrm{e} 40$ & $4.5 \mathrm{e} 41$ & -4.90 & -4.20 \\
\hline 26 & VV $705 \mathrm{~N}$ & $8.34 \pm 0.76$ & $1.39 \pm 0.32$ & $-0.71 \pm 0.10$ & $2.6 \mathrm{e}-14$ & $1.6 \mathrm{e}-14$ & $9.7 \mathrm{e} 40$ & $6.8 \mathrm{e} 40$ & -4.46 & -4.62 \\
\hline 26 & VV $705 \mathrm{~S}$ & $1.03 \pm 0.27$ & $0.06 \pm 0.07$ & $-0.90 \pm 0.34$ & $3.5 \mathrm{e}-15$ & $2.1 \mathrm{e}-15$ & $1.3 \mathrm{e} 40$ & $8.6 \mathrm{e} 39$ & - & - \\
\hline 27 & $\mathrm{~F} 15250+3608$ & $2.78 \pm 0.54$ & $0.00 \pm 0.18$ & $-1.0 \pm 0.30$ & $8.7 \mathrm{e}-15$ & $<4.2 \mathrm{e}-15$ & $6.2 \mathrm{e} 40$ & $<4.5 \mathrm{e} 40$ & -4.87 & $<-5.11$ \\
\hline 28 & UGC 9913 & $21.90 \pm 0.70$ & $4.68 \pm 0.46$ & $-0.65 \pm 0.04$ & $2.7 \mathrm{e}-14$ & $6.6 e-14$ & $2.0 \mathrm{e} 40$ & $6.8 \mathrm{e} 40$ & -5.56 & -5.03 \\
\hline 30 & NGC 6240 & $209.55 \pm 2.45$ & $59.57 \pm 1.34$ & $-0.56 \pm 0.01$ & $6.1 \mathrm{e}-13$ & $1.2 \mathrm{e}-12$ & $8.1 \mathrm{e} 41$ & $2.1 \mathrm{e} 42$ & -3.19 & -2.97 \\
\hline 32 & F17207-0014 & $7.13 \pm 0.40$ & $2.70 \pm 0.26$ & $-0.45 \pm 0.05$ & $1.9 \mathrm{e} 014$ & $3.9 \mathrm{e}-14$ & $9.9 \mathrm{e} 40$ & $2.2 \mathrm{e} 41$ & -5.04 & -4.70 \\
\hline 38 & ESO 286-IG19 & $14.83 \pm 0.58$ & $1.98 \pm 0.24$ & $-0.77 \pm 0.05$ & $4.3 \mathrm{e}-14$ & $3.3 e-14$ & $2.1 \mathrm{e} 41$ & $2.1 \mathrm{e} 41$ & -4.32 & -4.32 \\
\hline 42 & ESO 148-IG2 Tot & $14.46 \pm 0.56$ & $6.56 \pm 0.43$ & $-0.38 \pm 0.04$ & $4.0 \mathrm{e}-14$ & $1.1 \mathrm{e}-14$ & $1.9 \mathrm{e} 41$ & $8.3 \mathrm{e} 41$ & -4.36 & -3.72 \\
\hline
\end{tabular}


Table 3. continued.

\begin{tabular}{|c|c|c|c|c|c|c|c|c|c|c|}
\hline No. & Galaxy & $\begin{array}{l}S X \\
(1)\end{array}$ & $\begin{array}{l}H X \\
(2)\end{array}$ & $\begin{array}{l}H R \\
\text { (3) }\end{array}$ & $\begin{array}{c}F_{\text {SX }} \\
(4)\end{array}$ & $\begin{array}{c}F_{\mathrm{HX}} \\
(5)\end{array}$ & $\begin{array}{c}L_{S X} \\
(6)\end{array}$ & $\begin{array}{c}L_{\mathrm{HX}} \\
(7)\end{array}$ & $\begin{array}{c}S X / I R \\
(8)\end{array}$ & $\begin{array}{c}H X / I R \\
(9)\end{array}$ \\
\hline 42 & ESO 148-IG2 N & $2.78 \pm 0.24$ & $0.41 \pm 0.11$ & $-0.74 \pm 0.10$ & $7.6 e-15$ & $4.2 \mathrm{e}-15$ & $3.7 \mathrm{e} 40$ & $2.4 \mathrm{e} 40$ & -4.47 & -4.66 \\
\hline 42 & ESO 148-IG2 S & $4.44 \pm 0.30$ & $5.48 \pm 0.34$ & $+0.11 \pm 0.05$ & $1.3 e-14$ & $9.0 \mathrm{e}-14$ & $5.7 \mathrm{e} 40$ & $6.6 \mathrm{e} 41$ & -4.75 & -3.69 \\
\hline 44 & F23365+3604 & $1.67 \pm 0.41$ & $1.07 \pm 0.33$ & $-0.22 \pm 0.20$ & $5.7 e-15$ & $1.1 \mathrm{e}-14$ & $5.3 \mathrm{e} 40$ & $1.6 \mathrm{e} 41$ & -5.06 & -4.58 \\
\hline
\end{tabular}

Notes. Column (1): background corrected count rate in the $0.5-2 \mathrm{keV}$ band in unit of $10^{-3} \mathrm{ct} \mathrm{s}^{-1}$. Column (2): background corrected count rate in the $2-8 \mathrm{keV}$ band in unit of $10^{-3} \mathrm{ct} \mathrm{s}^{-1}$. Column (3): X-ray colour, as defined by $H R=(H-S) /(H+S)$. Column (4): observed $0.5-2 \mathrm{keV}$ band flux (erg s $\mathrm{cm}^{-2}$ ). Column (5): observed 2-7 keV band flux $\left(\mathrm{erg} \mathrm{s}^{-1} \mathrm{~cm}^{-2}\right.$ ). Column (6): 0.5-2 keV luminosity corrected for Galactic absorption ( $\mathrm{erg} \mathrm{s}^{-1}$ ). Column (7): 2-10 keV luminosity corrected for Galactic absorption (erg s${ }^{-1}$ ). Column (8): logarithmic luminosity ratio of the 0.5-2 keV and $8-1000 \mu \mathrm{m}$ bands. Column (9): logarithmic luminosity ratio of the $2-10 \mathrm{keV}$ and $8-1000 \mu \mathrm{m}$ bands.

AGN. Other examples are F18293-3413, VV 340 N, UGC 9913 (hereafter Arp 220), NGC 6240 and ESO 148-IG2.

\subsection{Flux density spectra}

X-ray spectra are traditionally shown as count rate spectra, i.e., data folded through the detector response. However, in this paper, in the interest of comparing the X-ray spectra with other multi-wavelength datasets in GOALS, the ACIS spectra have been corrected for the detector response curve and further converted into flux density units (Fig. 4). This correction may introduce some uncertainty, particularly when a spectral bin is wide within which the response varies rapidly, e.g., at the high energy end of the bandpass. In spite of this caveat, we think that this presentation has merit given that the spectral properties can be directly observed without resorting to spectral fitting. For this purpose, the flux density range for all spectra was also kept to be 2 orders of magnitude except where the dynamic range of the spectra exceeds 2 decades. The flux density is in units of $\left[10^{-14} \mathrm{erg} \mathrm{cm}^{-2} \mathrm{~s}^{-1} \mathrm{keV}^{-1}\right]$, and can readily be converted into units of $\left[\mathrm{W} \mathrm{m}^{-2} \mathrm{~Hz}^{-1}\right.$ ] by multiplying by $4.17 \times 10^{-35}$.

For some objects, data of spatially separate components are shown in Fig. 4, and when we consider it appropriate, data for the total emission are shown to help facilitate comparison with spectra taken from other X-ray observatories at lower spatial resolution. Finally, we note that these are not "unfolded spectra", which are sometimes presented in the literature. Instead, they have been corrected solely for the detector effective area while preserving the energy resolution of the detector, and thus are independent of any spectral model which might be fitted. (Note: The spectra shown in Fig. 4 are for display purposes only; all physical quantities reported in this paper were obtained through conventional spectral fitting to the count rate spectra with appropriate detector responses.)

\subsection{X-ray colour and AGN selection}

The X-ray colour, or hardness ratio, $H R$, gives the relative strength of the X-ray emission above and below $2 \mathrm{keV}$ (in counts). Since strong emission above $2 \mathrm{keV}$ is often associated with an absorbed X-ray source with a column density, $N_{\mathrm{H}}$, in the range of $10^{22}-10^{24} \mathrm{~cm}^{-2}$, which, in turn indicates the presence of an obscured AGN, it often serves as a crude probe of AGN.

As described in Iwasawa et al. (2009), AGN are selected as follows: The primary criterion is an hard X-ray spectrum as assessed by the X-ray colour, $H R$. The values of $H R$ as a function of $L_{\text {ir }}$ are plotted in Fig. 5. The median value of $H R$ is -0.56 . Objects with $H R>-0.3$ are classified as an AGN. This threshold is chosen because ULIRGs known to host AGN (e.g. Mrk 231,
UGC 8696 (=Mrk 273), UGC 5101) cluster just above this value. All of the optically identified AGN, i.e., Seyfert 1 and Seyfert 2 galaxies, in our sample are selected by this criterion.

Some Compton-thick AGN are missed by a $H R>-0.3$ selection because of their weakness in the hard band given that only reflected radiation is observed. The relative strength of the hard X-ray emission is largely suppressed, giving a small value for $H R$. Objects that show a strong Fe $\mathrm{K}$ line at $6.4 \mathrm{keV}$, a characteristic signature of a Compton-thick AGN, are also classified as AGN (e.g. NGC 6240 - Iwasawa \& Comastri 1998; Vignati et al. 1999; Ikebe et al. 2000; Komossa et al. 2003; NGC 3690 West - Della Ceca et al. 2002; Ballo et al. 2004; VV 340a - Armus et al. 2009; UGC 5101 - Imanishi et al. 2003). Note that highionization Fe $\mathrm{K}$ lines from Fe xxv or Fe xxvI at $6.7-7 \mathrm{keV}$ that have been found in a few objects, (e.g., NGC 3690 East - Ballo et al. 2004; Arp 220 - Iwasawa et al. 2005), are not considered here as evidence for AGN since they can also originate from hot gas produced by a starburst.

The selection by $H R$ and the $6.4 \mathrm{keV} \mathrm{Fe} \mathrm{K} \mathrm{line} \mathrm{finds} 16 \mathrm{ob}-$ jects that contain AGN. The remaining objects are characterized by relatively soft spectra, defined by small $H R$ values or relative weakness of the hard X-ray band emission (2-8 keV). The integral spectral properties of this sample of 29 "hard X-ray quiet" (HXQ, as defined by small $H R$ ) objects are reported in a separate paper (Iwasawa et al. 2009), in which the detection and origin of a high-ionization Fe $\mathrm{K}$ line in the integrated spectrum is discussed. The X-ray classifications of the sample are given in Table 5, along with optical and SDSS spectral types, the mid-IR [Nev] $\lambda 14.3 \mu \mathrm{m}$ detection, and the X-ray AGN selection criteria, which are met. We note that ESO 286-IG19 is classified as AGN in Franceschini et al. (2003), based on the XMM-Newton data (see Appendix A for more detail), while neither our Xray or mid-IR criteria selected this object as an AGN. Although ESO 286-IG19 remains as a viable AGN candidate, the discussion below assume that this object is a HXQ.

In general, AGN being powerful X-ray emitters, an X-ray observation is a sensitive probe of AGN unless they are hidden behind Compton thick obscuration. However, it is not necessarily true that AGN selected by the X-ray technique are energetically important for the bolometric output of LIRGs. Here we have made an attempt to access the importance of AGN contribution to the IR luminosity of the objects hosting the X-ray selected AGN, using the observed X-ray properties. However the uncertainty in these estimate should be quite large, as explained below, and they can be considered as a guide only.

There are two steps for estimating the bolometric luminosity of an AGN from the observed X-ray luminosity: 1) absorption correction which recovers the instrinsic X-ray luminosity by removing the flux suppression effect due to absorption; 


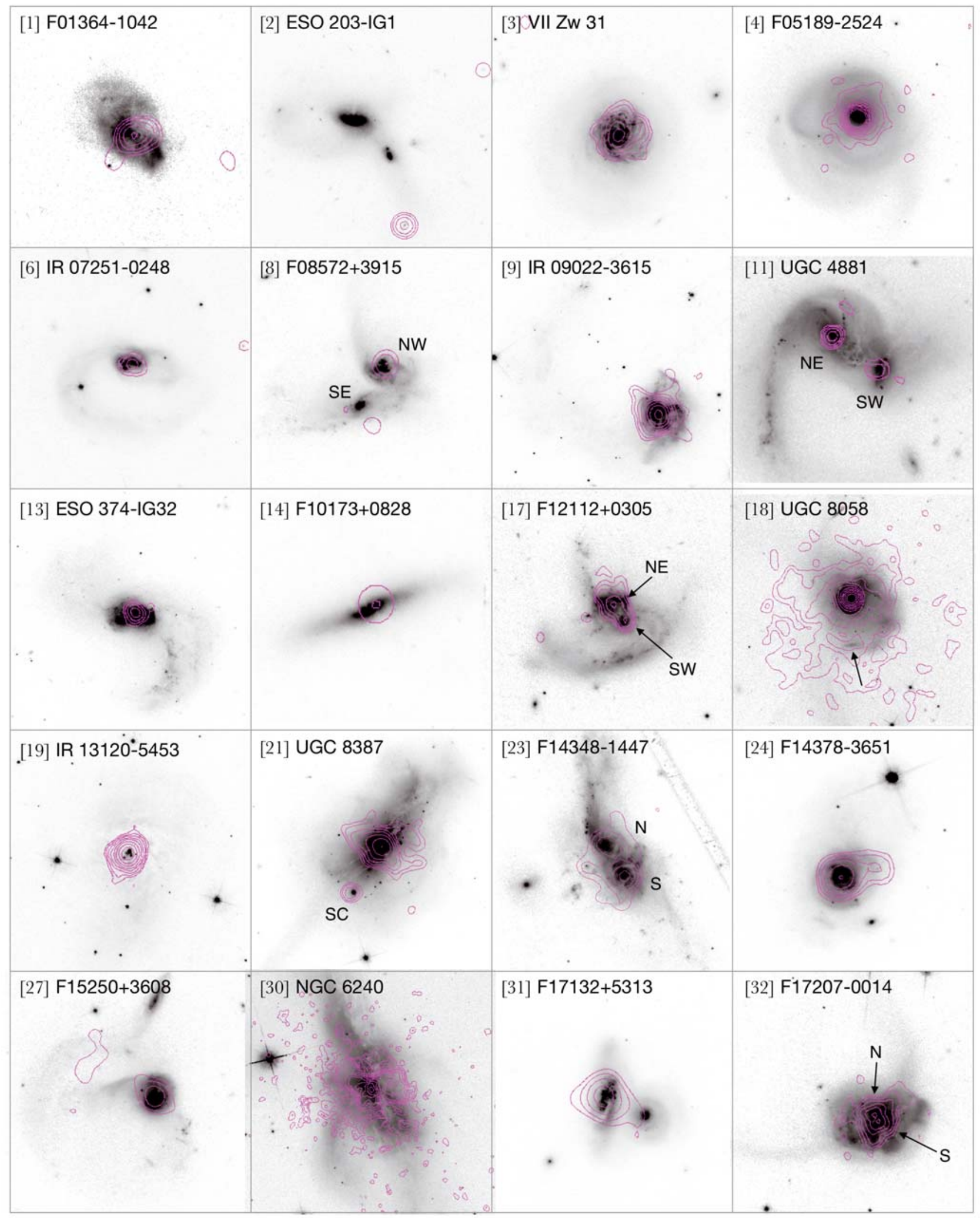

Fig. 2. X-ray and optical images of the 44 C-GOALS sample galaxies. X-ray (0.4-7 keV) brightness contours (magenta) are overlaid on the HSTACS F814W image (grey scale) for each object. The orientation of all images is north up and east to the left. Eleven contour levels are defined in the fixed surface brightness range $4 \times 10^{-5}-7 \times 10^{-3}$ [counts s arcsec $^{-2}$ ], which is divided into ten equal logarithmic intervals. For majority of objects, these contour levels were applied. For nine objects (F10173+0828, F17132+5313, F19297-0406, CGCG 448-020, F01364-1042, IRAS 21101+5810, F10565+2448, UGC 9913, and UGC 5101), a further lower interval was added to outline lower surface brightness features. For four bright objects, (UGC 8696, NGC 3690, F05189-2524 and UGC 8058), custom contour levels were made for describing their X-ray morphology - these are listed in Table 4. 


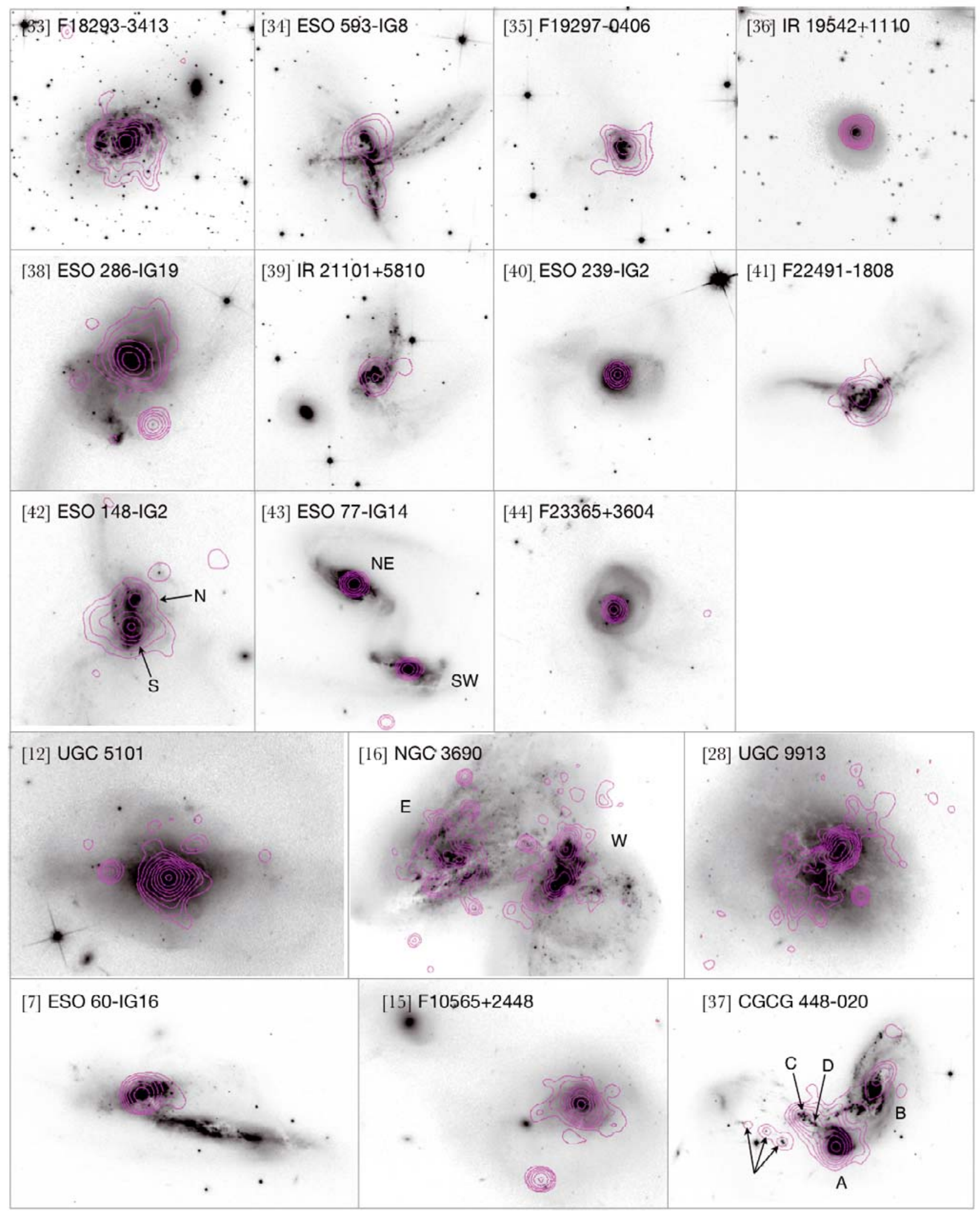

Fig. 2. continued.

2) bolometric correction which converts the absorptioncorrected X-ray luminosity to the bolometric luminosity, assuming a typical spectral energy distribution (SED) of an unobscured AGN (e.g., Elvis et al. 1994). In our rough estimates, the absorption correction factors of 3 for a Compton thin AGN and 100 for a Compton thick AGN (i.e., $1 \%$ of $2-10 \mathrm{keV}$ emission from a hidden AGN is visible) are adopted for the $2-10 \mathrm{keV}$ band. The bolometric correction is assumed to be 


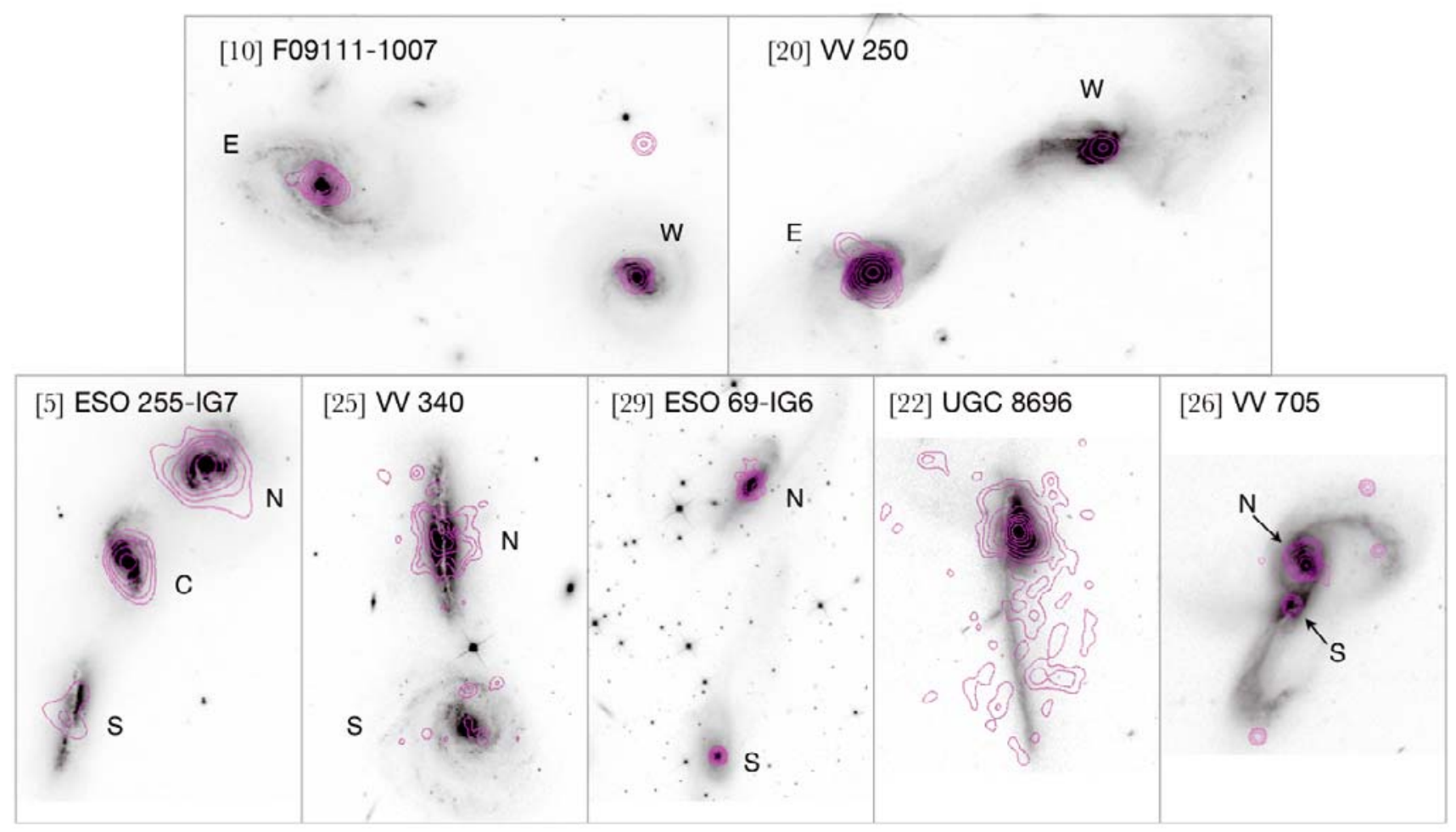

Fig. 2. continued.

\section{UGC 8387}
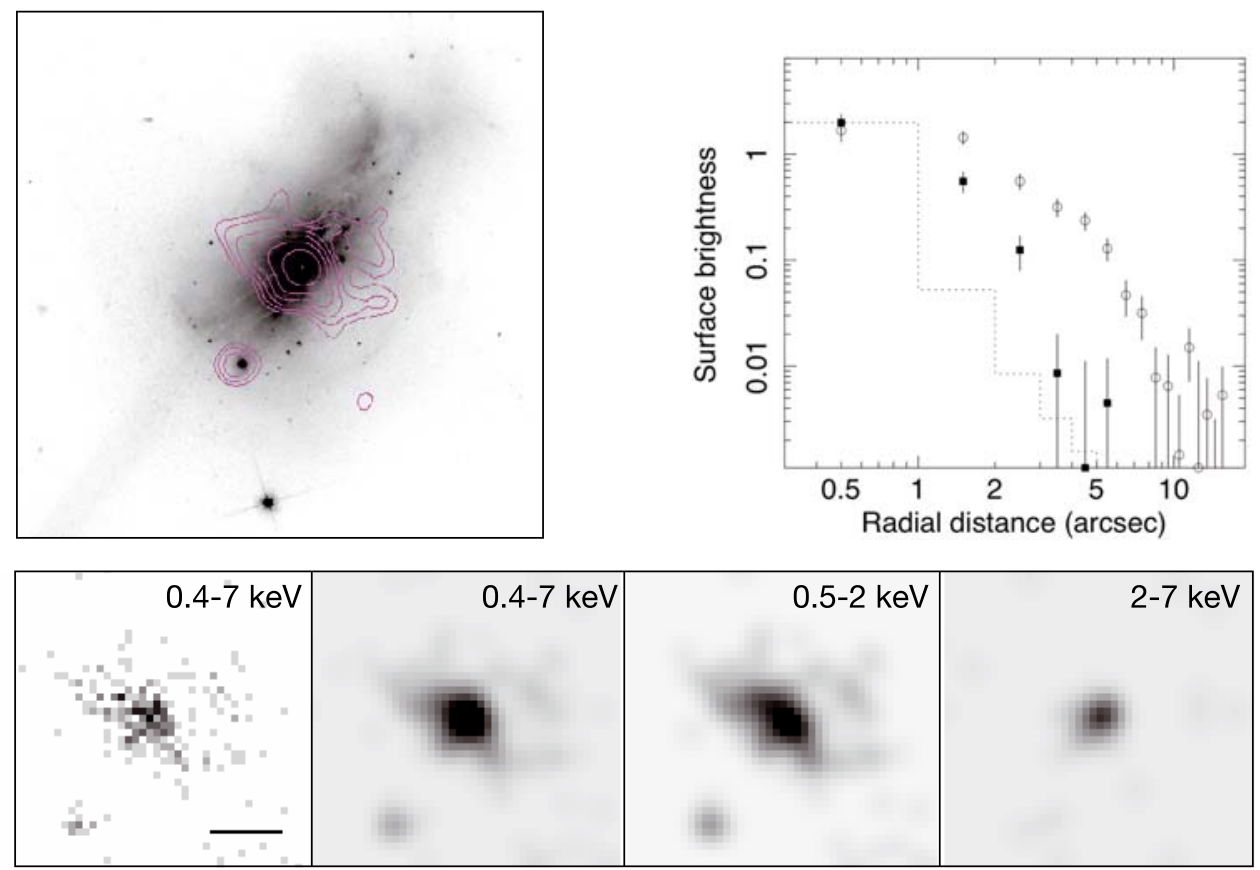

Fig. 3. An example of X-ray images and the surface brightness distributions for individual objects is presented. The object shown here is UGC 8387 (=IC 883). Similar multi-panel figures for the other 43 objects in our C-GOALS sample can be found in Appendix. Upper left: the X-ray (0.4$7 \mathrm{keV}$ ) brightness contours overlaid on the HST ACS I-band image. Upper right: the radial surface brightness profiles in the 0.5-2 keV (open circles) and 2-7 keV (filled squares) bands are shown. These profiles are the azimuthal average and are measured from the peak of 2-7 keV emission at 1 arcsec resolution. The Point Spread Function (PSF) computed at $3 \mathrm{keV}$ is shown as a dotted histogram where the peak has been matched to the observed 2-7 keV peak for comparison. The bottom four-panel figure shows (left to right) the raw 0.4-7 keV image, the Gaussian smoothed version of the same image, the $0.5-2 \mathrm{keV}$ smoothed image, and the $2-7 \mathrm{keV}$ smoothed image. The pixel size is $\approx 0.5^{\prime \prime} \times 0.5^{\prime \prime}$. The scale bar indicates 5 arcsec. 
A\&A 529, A106 (2011)
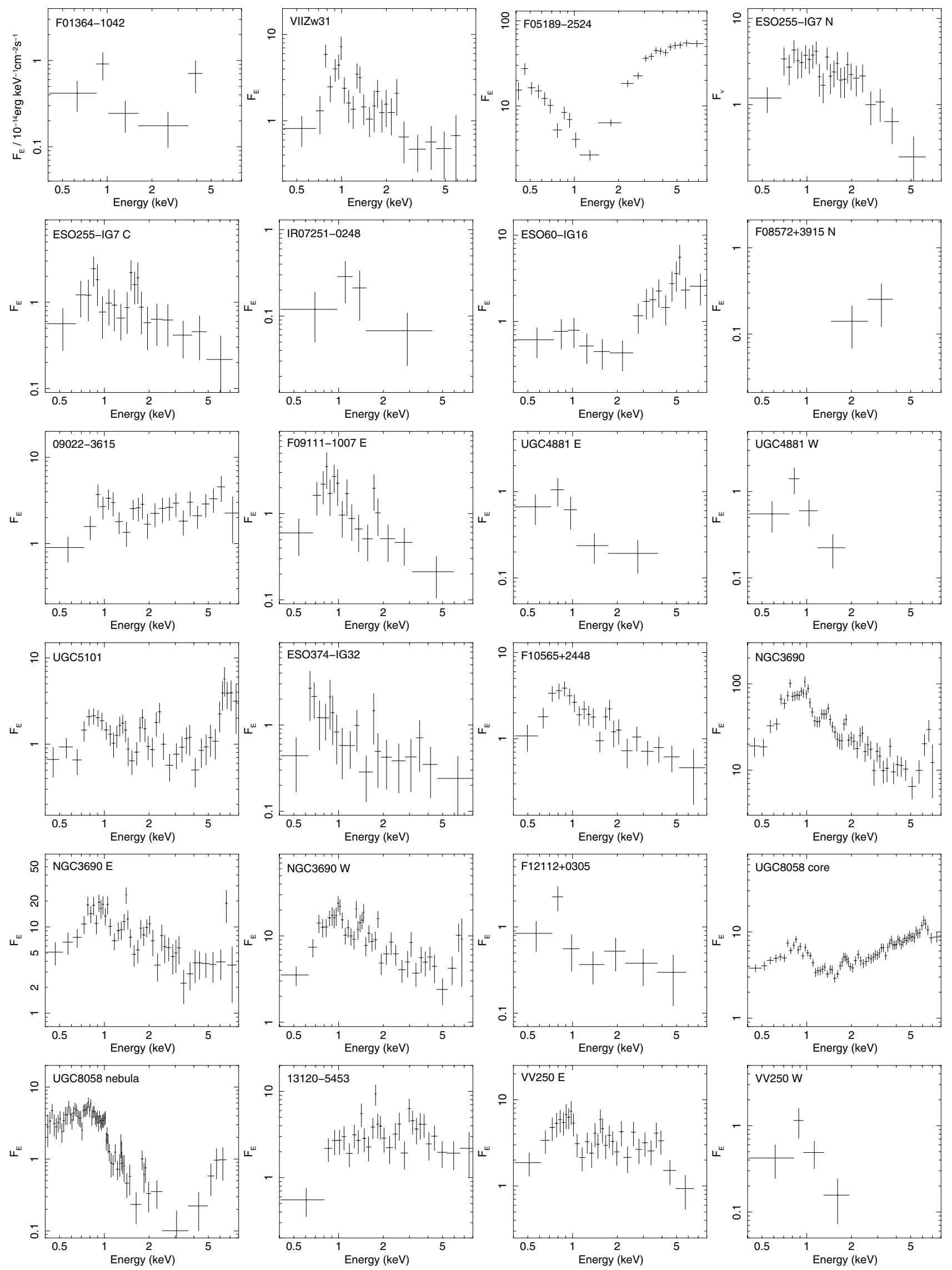

Fig. 4. The X-ray flux density spectra of the C-GOALS sources, obtained from the Chandra ACIS. The energy scale is as observed. The flux density is in units of $\left[10^{-14} \mathrm{erg} \mathrm{s}^{-1} \mathrm{~cm}^{-2} \mathrm{keV}^{-1}\right]$. 
K. Iwasawa et al.: C-GOALS survey
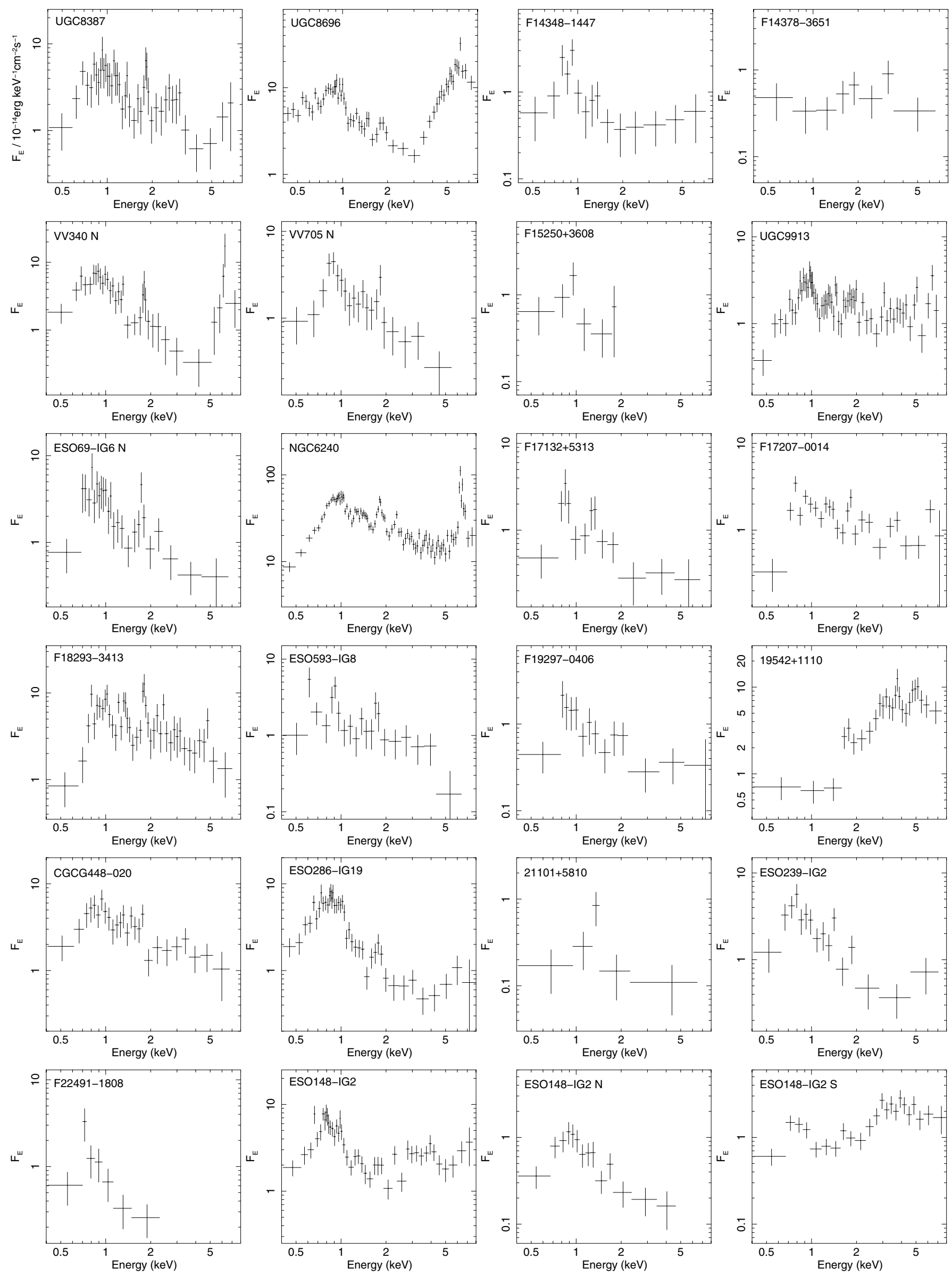

Fig. 4. continued. 

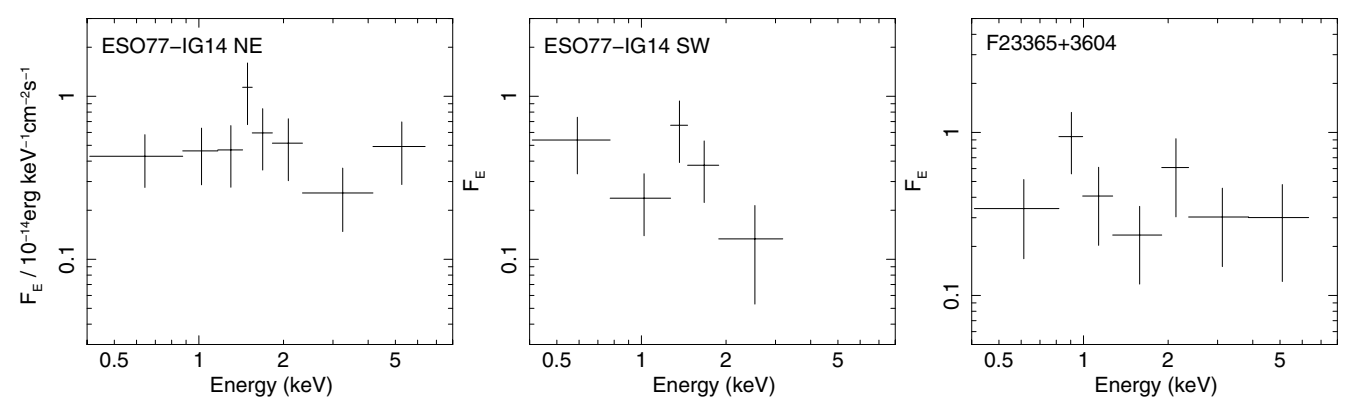

Fig. 4. continued.

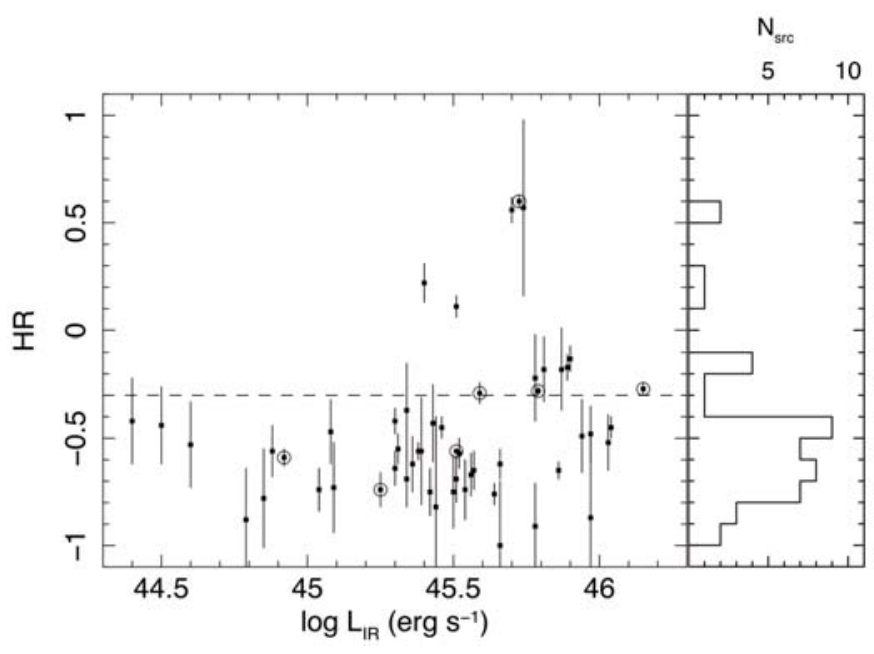

Fig. 5. The X-ray colour $(H R)$ as a function of infrared luminosity and its distribution. See the text for the definition of $H R$. While the $H R$ is derived for individual components in a single object when the X-ray sources are well resolved into multiple components (i.e., the nuclear separation is $\sim 4^{\prime \prime}$ or larger; see Table 3 ). The threshold for selecting AGN, based on the $H R>-0.3$, is indicated by the dashed line. Objects in which a $6.4 \mathrm{keV} \mathrm{Fe} \mathrm{K} \alpha$ line detetion has been reported are marked by open circles. Three known Compton thick AGN with strong $(6.4 \mathrm{keV})$ Fe K (NGC 3690 W, NGC 6240, VV340 N) lie below the HR threshold. The $H R$ distribution histogram of $H R$ is attached on the right hand side. The median is $H R=-0.56$.

Table 4. X-ray contours for bright objects.

\begin{tabular}{rlccc}
\hline \hline No. & Galaxy & $\begin{array}{c}\text { Low } \\
(1)\end{array}$ & $\begin{array}{c}\text { High } \\
(2)\end{array}$ & $\begin{array}{c}n \\
(3)\end{array}$ \\
\hline 4 & F05189-2524 & $3.0 \times 10^{-5}$ & $4.2 \times 10^{-3}$ & 10 \\
16 & NGC 3690 & $2.7 \times 10^{-5}$ & $1.8 \times 10^{-3}$ & 10 \\
18 & UGC 8058 & $8.5 \times 10^{-7}$ & $3.7 \times 10^{-4}$ & 11 \\
22 & UGC 8696 & $2.3 \times 10^{-6}$ & $1.8 \times 10^{-3}$ & 9 \\
\hline
\end{tabular}

Notes. Column (1): the lowest contour (cts $\left.\mathrm{s}^{-1} \operatorname{arcsec}^{-2}\right)$ in Fig. 2. Column (2): the highest contour (cts s$\left.{ }^{-1} \operatorname{arcsec}^{-2}\right)$ in Fig. 2. Column (3): the number of contour levels in Fig. 2.

$L_{\text {bol }} / L_{2-10}=30$, where $L_{2-10}$ is absorption-corrected $2-10 \mathrm{keV}$ luminosity, which is adopted from Marconi et al. (2004) for objects with $L_{\text {bol }} \sim 10^{12} L_{\odot}$.

When applying the absorption correction, there is an ambiguity inherent in the AGN selected by X-ray colour alone with a poor quality spectrum. They show flat X-ray spectra (Fig. 4), as the $H R$ analysis infers, but it is not trivial whether they are Compton thick or thin objects without a good constraint on a $\mathrm{Fe} \mathrm{K}$ line. As the respective absorption correction factors differ significantly, the classification of the obscuration type introduces a large uncertainty in the AGN luminosity. Since only weak reflected light is observed from a Compton thick AGN, we tentatively assume that the X-ray colour selected AGN with $\log \left(L_{\mathrm{HX}} / L_{\mathrm{ir}}\right)<-4$ are Compton thick AGN candidates ${ }^{2}$ and they are marked as such in Table 6. It is also noted that AGN with a reasonable quality spectrum showing a low-energy cut-off due to moderate absorption, $N_{\mathrm{H}} \sim 10^{22}-10^{23} \mathrm{~cm}^{-2}$, [F05189-2524, ESO 60-IG16, Mrk 273, 19542+1110] all have larger values between -3.4 and -2.5 in $\log \left(L_{\mathrm{HX}} / L_{\mathrm{ir}}\right)$.

The absorption correction for a Compton thick AGN is also rather uncertain, since it strongly depends on the geometry of the obscuring matter, which is not well known. While a uniform correction factor of $\times 100$ is used here, the final error in $F_{\text {agn }}$ can be easily larger than $0.5 \mathrm{dex}$, as demonstrated by $F_{\text {agn }}>1$ for NGC 6240. In any case, taking the face values, the median of $\log F_{\text {agn }}$ is -1 , and there are only two objects (Mrk 231 and NGC 6240) in which the AGN contribution exceeds $50 \%$ of their infrared luminosity. In summary, according to this simplistic estimate, AGN-dominated objects would seem to be in the minority in our sample, and the typical AGN contribution to the infrared luminosity would appear to be $\sim 10 \%$.

The X-ray-selected AGN represent $37 \%$ of the total sample (16/44). This figure is comparable to that inferred from the midIR diagnostics for the same IR luminosity range (Petric et al. 2011). When the sample is divided into two luminosity bins, above and below the median IR luminosity of $\log \left(L_{\mathrm{ir}} / L_{\odot}\right)=$ $11.99,12$ of these $16 \mathrm{AGN}(75 \%)$ are above the median, in agreement with previous studies showing the fraction of AGN rising with increasing $L_{\text {ir }}$ (e.g., Veilleux et al. 1995). If objects with $[\mathrm{Nev}] \lambda 14.3 \mu \mathrm{m}$ detection (Table 5), which is generally considered as evidence for AGN, are included, the AGN fraction would increase up to $48 \%$. In terms of merger stage, X-ray-selected AGN tend to be found more in mergers of later stages. For example, 50\% (9/18) of final stage mergers (nuclear separation $<1 \mathrm{kpc}$ ) are X-ray-seleted AGN, while only $26 \%$ (7/27) of earlier stage mergers are X-ray selected AGN. We refer to Evans et al. (in prep.) for details on the galaxy morphology and merger-stage classifications based on our HST imaging.

NGC 6240 is the only object in the sample where two nuclei both show evidence for harboring a powerful $\mathrm{AGN}^{3}$ out of 24 objects that have double or tripple nuclei. It should be noted

2 The hard X-ray to [OIII] $\lambda 5007$ ratio is often used as a diagnostic for a Compton thick AGN (e.g., Bassani et al. 1999). However, this diagnostic may fail for dusty objects like LIRGs because emission lines from the narrow-line regions are likely suppressed as well.

${ }^{3}$ Mrk 266 (Mazzarella et al. 2010) and Mrk 463 (Bianchi et al. 2008), two GOALS galaxies, but with $L_{\text {ir }}$ below our current C-GOALS sample threshold, are other objects which are found to have X-ray evidence for AGN in both nuclei. 
Table 5. Classification of C-GOALS sources.

\begin{tabular}{|c|c|c|c|c|c|c|}
\hline No. & Object & $\begin{array}{l}\mathrm{VO} \\
\text { (1) }\end{array}$ & $\begin{array}{c}\text { YKS } \\
(2)\end{array}$ & $\begin{array}{c}D_{\text {agn }} \\
\text { (3) }\end{array}$ & $\begin{array}{c}\mathrm{Ne} \mathrm{v]} \\
\text { (4) }\end{array}$ & $\begin{array}{l}X_{\text {agn }} \\
(5)\end{array}$ \\
\hline \multicolumn{7}{|c|}{ X-ray selected AGN } \\
\hline 4 & F05189-2524 & S2 & S2 & 1 & Y & CL \\
\hline 7 & ESO 60-IG16 & - & - & - & - & $\mathrm{C}$ \\
\hline 8 & F08572+3915 NW & $\mathrm{L}$ & S2 & 0.6 & $\mathrm{~N}$ & $\mathrm{C}$ \\
\hline 9 & 09022-3615 & - & - & - & $\mathrm{N}$ & $\mathrm{C}$ \\
\hline 12 & UGC 5101 & $\mathrm{~L}$ & S2 & 0.6 & $\mathrm{Y}$ & CL \\
\hline 16 & NGC $3690 \mathrm{~W}$ & $\mathrm{H}$ & - & - & - & $\mathrm{L}$ \\
\hline 18 & UGC 8058 & S1 & S1 & 1 & $\mathrm{~N}$ & CL \\
\hline 19 & $13120-5453$ & - & - & - & Y & $\mathrm{C}$ \\
\hline 22 & UGC 8696 & S2 & S2 & 0.8 & Y & $\mathrm{CL}$ \\
\hline 23 & F14348-1447 SW & $\mathrm{L}$ & $\mathrm{cp}$ & 0.7 & $\mathrm{~N}$ & $\mathrm{C}$ \\
\hline 24 & F14378-3651 & S2 & - & - & $\mathrm{N}$ & $\mathrm{C}$ \\
\hline 25 & VV $340 \mathrm{~N}$ & $\mathrm{~L}$ & $\mathrm{cp}$ & 0.5 & Y & $\mathrm{L}$ \\
\hline 30 & NGC 6240 & $\mathrm{~L}$ & $\mathrm{~L}$ & 0.8 & Y & $\mathrm{L}$ \\
\hline 36 & $19542+1110$ & - & - & - & - & $\mathrm{C}$ \\
\hline 42 & ESO 148-IG2 & - & - & - & - & $\mathrm{C}$ \\
\hline 44 & F23365+3604 & $\mathrm{L}$ & $\mathrm{cp}$ & 0.3 & $\mathrm{~N}$ & $\mathrm{C}$ \\
\hline \multicolumn{7}{|c|}{ Others (HXQ sample) } \\
\hline 1 & F01364-1042 & $\mathrm{L}$ & $\mathrm{L}$ & 0.8 & - & \\
\hline 2 & ESO 203-IG1 & - & - & - & - & \\
\hline 3 & VII Zw 31 & $\mathrm{H}$ & - & - & Y & \\
\hline 5 & ESO 255-IG7 & - & $\mathrm{H}$ & 0 & - & \\
\hline 6 & $07251-0248$ & - & - & - & - & \\
\hline 10 & F09111-1007 & - & - & - & Y & \\
\hline 11 & UGC $4881 \mathrm{SW} / \mathrm{NE}$ & $\mathrm{H} / \mathrm{H}$ & $\mathrm{cp} / \mathrm{cp}$ & $0.3 / 0.2$ & - & \\
\hline 13 & ESO 374-IG32 & - & $\mathrm{cp}$ & 0.3 & - & \\
\hline 14 & F10173+0828 & - & - & - & - & \\
\hline 15 & $F 10565+2448$ & $\mathrm{H}$ & $\mathrm{cp}$ & 0.2 & $\mathrm{~N}$ & \\
\hline 16 & NGC 3690 E & $\mathrm{H}$ & - & - & - & \\
\hline 17 & $\mathrm{~F} 12112+0305$ & $\mathrm{~L}$ & S2 & 0.7 & $\mathrm{~N}$ & \\
\hline 20 & VV $250 \mathrm{NW} / \mathrm{SE}$ & $\mathrm{H} / \mathrm{H}$ & $\mathrm{cp} / \mathrm{cp}$ & $0.4 / 0.3$ & - & \\
\hline 21 & UGC 8387 & $\mathrm{~L}$ & $\mathrm{cp}$ & 0.5 & Y & \\
\hline 26 & VV 705 S/N & $\mathrm{L} / \mathrm{H}$ & $\mathrm{cp} / \mathrm{cp}$ & $0.5 / 0.3$ & - & \\
\hline 27 & F15250+3608 & $\mathrm{L}$ & cp & 0.4 & $\mathrm{~N}$ & \\
\hline 28 & UGC 9913 & $\mathrm{~L}$ & $\mathrm{~L}$ & 0.7 & $\mathrm{~N}$ & \\
\hline 29 & ESO 69-IG6 & - & - & - & - & \\
\hline 31 & $\mathrm{~F} 17132+5313$ & $\mathrm{H}$ & $\mathrm{H}$ & 0 & Y & \\
\hline 32 & F17207-0014 & $\mathrm{H}$ & $\mathrm{H}$ & 0 & $\mathrm{~N}$ & \\
\hline 33 & F18293-3413 & - & - & - & - & \\
\hline 34 & ESO 593-IG008 S/N & $\mathrm{L} / \mathrm{H}$ & $\mathrm{S} 2 / \mathrm{cp}$ & $0.4 / 0.2$ & Y & \\
\hline 35 & F19297-0406 & - & - & - & $\mathrm{N}$ & \\
\hline 37 & CGCG 448-020 & $\mathrm{H}$ & $\mathrm{H}$ & 0 & $\mathrm{~N}$ & \\
\hline 38 & ESO 286-IG19 & $\mathrm{H}$ & $\mathrm{H}$ & 0 & - & \\
\hline 39 & $21101+5810$ & - & - & - & - & \\
\hline 40 & ESO 239-IG2 & - & - & - & - & \\
\hline 41 & F22491-1808 & $\mathrm{H}$ & $\mathrm{H}$ & 0 & $\mathrm{~N}$ & \\
\hline 43 & ESO 77-IG14 & $\mathrm{H}$ & - & - & - & \\
\hline
\end{tabular}

Notes. Column (1): optical class based on Veilleux \& Osterbrock (1987) diagram from Veilleux et al. (1995, 1999). H: star-forming; S1: Seyfert 1; S2: Seyfert 2; L: LINER. Column (2): the SDSS class from Yuan et al. (2010). The symbols are the same as in Col. (1), plus an additional class of "composite" objects; cp: composite of starburst and AGN. Column (3): the AGN fraction $D_{\text {agn }}$ derived from $[\mathrm{O} \mathrm{I}] / \mathrm{H} \alpha$ diagram (see Yuan et al. 2010, for details). Column (4): detection of [Ne v] $14.32 \mu \mathrm{m}$ from Farrah et al. (2007). New results on CGCG 448020 (Inami et al. 2010), VV340 N (Armus et al. 2009), UGC 8387 (Modica et al. 2010), and other GOALS objects (Petric et al. 2011) are also included. Column (5): AGN selection criteria: C: X-ray colour $(H R \geq 0.3)$; L: $6.4 \mathrm{keV}$ Fe $\mathrm{K}$ line.

that we do not consider NGC 3690 to have a double AGN, as the Fe xxv line found in the eastern galaxy is not taken as an AGN signature. Other AGN are found either in a single nucleus

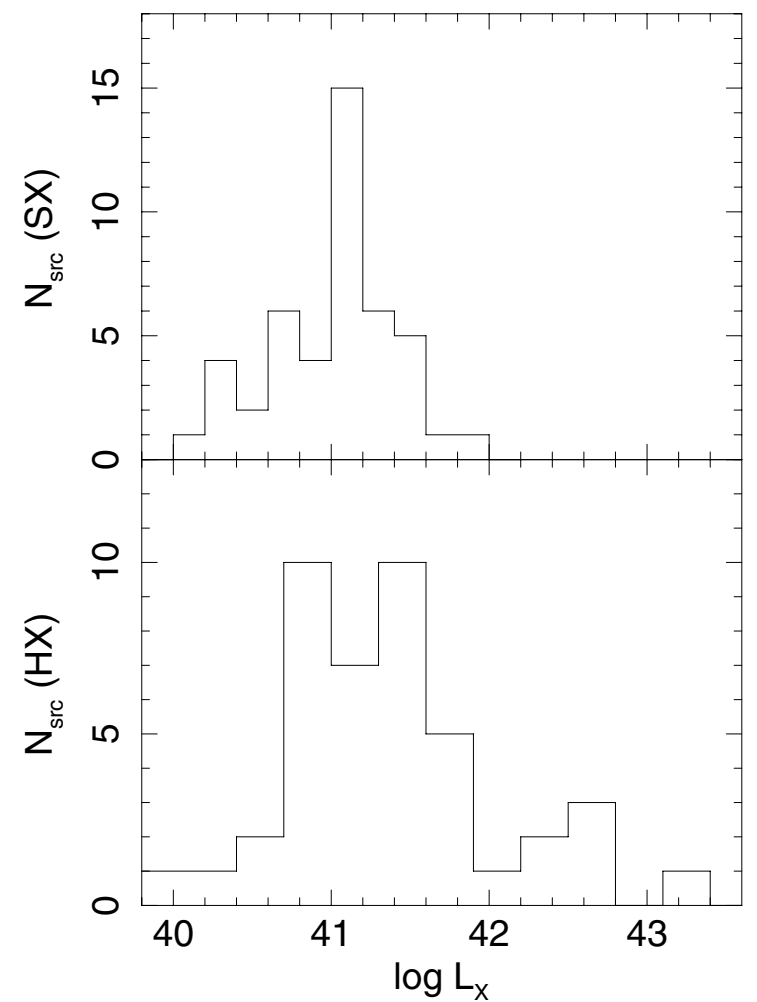

Fig. 6. X-ray luminosity distribution in the soft (0.5-2 keV; upper panel) and hard (2-10 keV; lower panel) bands.

( 8 objects) or in one member of a double nucleus system (7 objects).

\section{4. $X$-ray luminosities and correlation with $L_{\text {ir }}$}

The X-ray fluxes were estimated based on a best-fitting spectral model in the $0.5-2 \mathrm{keV}$ and $2-7 \mathrm{keV}$ bands (i.e., no correction of absorption). For faint sources, the hard band data are often not good enough to constrain the shape, in which case a power-law of $\Gamma=2.1$ is assumed to derive the $2-7 \mathrm{keV}$ flux (e.g., Ranalli et al. 2003). The X-ray luminosities are estimated by correcting only for Galactic absorption. (Note: Significant absorption intrinsic to the sources is likely present. However, X-ray spectra of our objects are complex with multiple components, while most of the spectra do not have sufficient quality to uniquely decompose and estimate absorption of each component. For this reason, we opt to present the luminosities as observed.) The hard band luminosity is for the $2-10 \mathrm{keV}$ band, which is estimated by extrapolating the $2-7 \mathrm{keV}$ spectrum. For a few objects, the luminosity values have been updated from those in Iwasawa et al. (2009). The distribution of the X-ray luminosity in the soft and hard bands is shown in Fig. 6. The luminosity distribution in both bands peaks at $\log \left(L_{\mathrm{X}}\right) \approx 41.1 \mathrm{erg} \mathrm{s}^{-1}$, but the hard band luminosity is spread over a wider range. The median logarithmic values for the soft and hard band luminosities are $41.1 \mathrm{erg} \mathrm{s}^{-1}$ and $41.3 \mathrm{erg} \mathrm{s}^{-1}$, respectively.

A moderate correlation between the IR and X-ray luminosities can be seen in Fig. 7, with a typical spread over an order of magnitude. Note that when multiple components are present in a single object, their luminosities are plotted separately in Fig. 7. When integrated luminosities in a single objects are used, as 

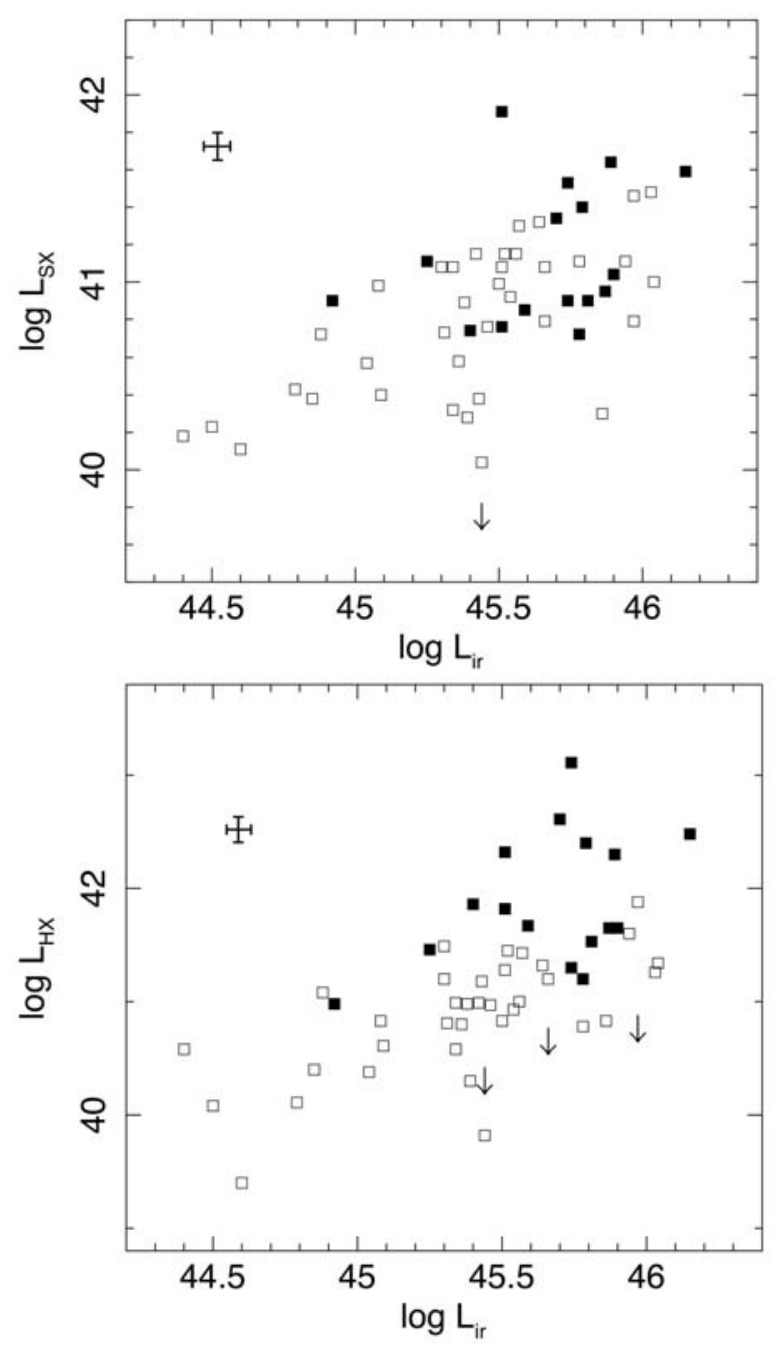

Fig. 7. Plots of X-ray (upper panel: $0.5-2 \mathrm{keV}$; lower panel: $2-10 \mathrm{keV}$ ) versus infrared luminosity. The X-ray luminosity has been corrected only for Galactic absorption. The X-ray selected AGN are marked with filled squares. The typical error bars are indicated. For objects that are not detected, the 95 per cent upper limits are indicated by arrows. When multiple components are resolved in a single system, their luminosities are computed separately (Table 3). The decomposition of the infrared luminosity in individual objects is described in Appendix A. There is a moderate correlation, with a correlation coefficient of $\sim 0.6$ for both plots.

shown in Iwasawa et al. (2009) ${ }^{4}$, the correlation becomes less clear. In the soft X-ray band, AGN are mixed in with the distribution of non-AGN (HXQ) objects, while in the hard X-ray band, AGN tend to be the more luminous X-ray sources, as expected.

The X-ray to IR luminosity ratio distribution shown in Fig. 8 has a significant spread, which is caused by the scatter around the correlation between the luminosities (e.g. Fig. 7) rather than any non-linear correlation. Typical values are $\log \left(L_{\mathrm{SX}} / L_{\mathrm{ir}}\right)=$ $-4.53 \pm 0.34$ for the soft X-rays and $\log \left(L_{\mathrm{HX}} / L_{\mathrm{ir}}\right)=-4.40 \pm 0.63$ for the hard X-rays (the uncertainties are the dispersion of the distributions). When X-ray-selected AGN are excluded, a linear relation between logarithmic X-ray and IR luminosities (Fig. 7) is given by $\log L_{\mathrm{SX}}=(-4.6 \pm 0.1)+\log L_{\text {ir }}$ for the soft X-ray

\footnotetext{
${ }^{4}$ In Iwasawa et al. (2009), the IR luminosity $(8-1000 \mu \mathrm{m})$ is also replaced by the FIR luminosity ( $40-400 \mu \mathrm{m})$ to allow direct comparison with the previous studies on the X-ray - FIR correlation.
}

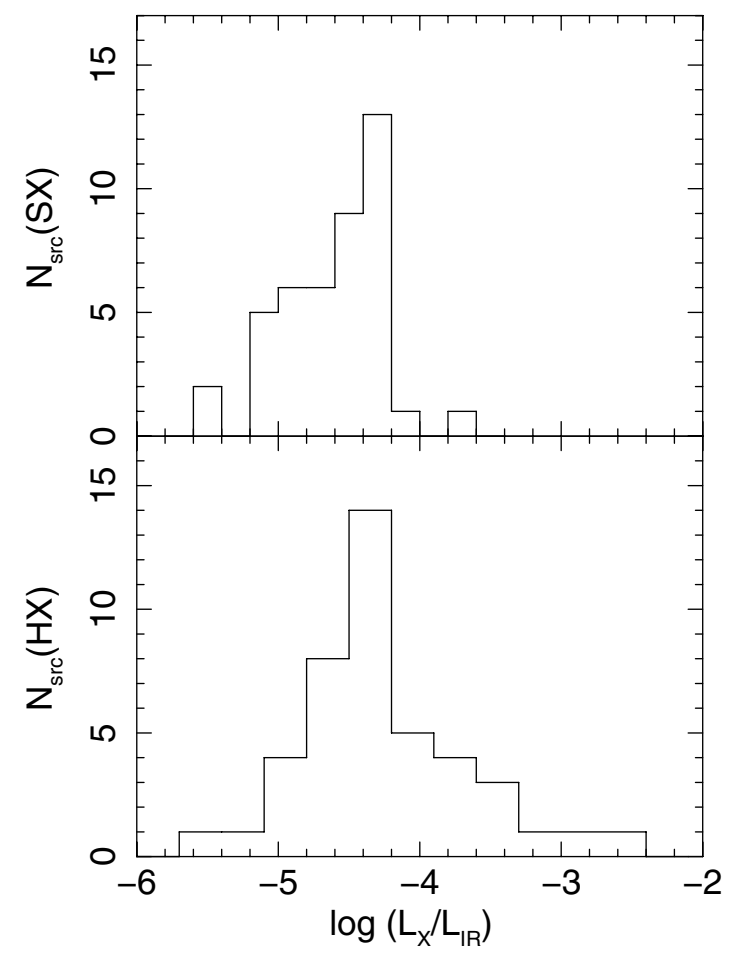

Fig. 8. X-ray to infrared luminosity ratio distribution.

band and $\log L_{\mathrm{HX}}=(-4.5 \pm 0.1)+\log L_{\text {ir }}$ for the hard X-ray band. These values are significantly lower than those found for local, star-forming galaxies with low star formation rates, e.g., $\log L_{\mathrm{X}} / L_{\mathrm{FIR}} \sim-3.7$ (Ranalli et al. 2003). Note that a direct comparison would need a correction for the different infrared bandpasses (details of the direct comparison can be found in Iwasawa et al. 2009).

\subsection{Radial surface brightness profiles}

Radial surface brightness profiles are derived in the soft $(0.5-$ $2 \mathrm{keV})$ and hard (2-7 keV) bands at a resolution of 1 arcsec. In general, the soft X-ray emission is spatially extended while the hard X-ray emission is compact. A few exceptions are sources dominated by absorbed hard X-ray emission as seen in objects like IRAS $19542+1110$, where both bands show equally compact emission.

With the $0.5^{\prime \prime}$ resolution of the Chandra ACIS, the presence of extended soft X-ray emission is immediately clear by visual inspection for all of the sources. To quantify the soft X-ray extension, azimuthally-averaged surface brightness profiles are produced in the soft $(0.5-2 \mathrm{keV})$ and hard (2-7 keV) bands separately for individual targets. An example of these surface brightness profiles is shown in Fig. 3 for UGC 8387, and those for all the other objects are presented in on-line material. The soft X-ray profiles show a range of shapes - from an exponetial profile to a more peaky, power-law type profile. Since not all of the sources have sufficient counts, the soft X-ray profiles are quantified by two characteristic radii, rather than fitting a profile model. The maximum extension radius, $r_{\max }$, and half power radius, $r_{\mathrm{hp}}$, are defined as follows: the radius where the surface brightness falls to $1 \%$ of the peak brightness (usually of the central bin of the surface brightness profile) is defined as $r_{\max }$ in arcsec. This radius gives a measure of the source extension, which has little dependence on the depth of the image. The total source count, $C_{\max }$, integrated within $r_{\max }$ is then derived. $C_{\max }$ can 
Table 6. Estimates of AGN contribution to IR luminosity.

\begin{tabular}{rlccc}
\hline \hline No. & Galaxy & $\begin{array}{c}\log (\mathrm{HX} / \mathrm{IR}) \\
(1)\end{array}$ & $\begin{array}{c}\text { CT } \\
(2)\end{array}$ & $\begin{array}{c}\log F_{\text {agn }} \\
(3)\end{array}$ \\
\hline 4 & F05189-2524 & -2.63 & & -0.65 \\
7 & ESO 60-IG16 & -2.54 & & -1.54 \\
8 & F08572+3915 & -4.44 & $*$ & -0.94 \\
9 & $09022-3615$ & -3.59 & & -1.59 \\
12 & UGC 5101 & -3.92 & $\circ$ & -0.42 \\
16 & NGC 3690 W & -4.07 & $\circ$ & -0.57 \\
18 & UGC 8058 & -3.67 & $\circ$ & -0.17 \\
19 & 13120-5453 & -4.25 & $*$ & -0.75 \\
22 & UGC 8689 & -3.39 & & -1.39 \\
23 & F14348-1447 S & -4.90 & $*$ & -1.50 \\
24 & F14378-3651 & -4.28 & $*$ & -0.78 \\
25 & VV 340 N & -3.86 & $\circ$ & -0.36 \\
30 & NGC 6240 & -2.97 & $\circ$ & +0.53 \\
36 & 19542+1110 & -3.05 & & -1.09 \\
42 & ESO 148-IG2 & -3.72 & & -1.72 \\
44 & F23365+3604 & -4.58 & $*$ & -1.08 \\
\hline
\end{tabular}

Notes. (1) Logarithmic luminosity ratio of the observed 2-10 keV and $8-1000 \mu \mathrm{m}$ bands, reproduced from Table 3. (2) Compton thick AGN with Fe K detection are marked as $\circ$. Candidate Compton thick AGN (see text for detail) are marked as *. (3) Logarithmic fraction of the $\mathrm{X}$-ray estimate for AGN contribution to the $8-1000 \mu \mathrm{m}$ luminosity.

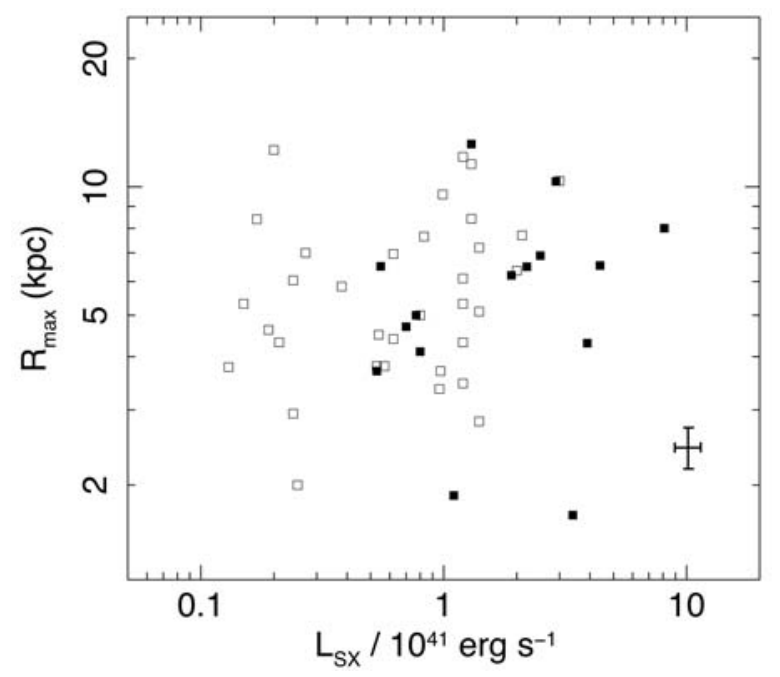

Fig. 9. The soft $\mathrm{X}$-ray emission radius, $R_{\max }(\mathrm{kpc})$ versus soft $\mathrm{X}$-ray luminosity. Filled symbols are of X-ray-selected AGN and open symbols are others. The typical error bars are indicated. No trend can be seen. The median value of $R_{\max }$ is $5.3 \mathrm{kpc}$.

differ from the total source count when the surface brightness, which is lower than $1 \%$ of the peak brightness, extends to large radii. The radius where the accumulated source count reaches half of $C_{\max }$ is defined as the half power radius, $r_{\mathrm{hp}}$, in arcsec. These quantities give a measure of the compactness/broadness of the source extension. Since both $r_{\max }$ and $r_{\mathrm{hp}}$ are determined as the innermost radial bin which satisfies the required condition, their uncertainty is always 0.5 arcsec. For a compact source (e.g., $r_{\mathrm{hp}} \sim 1^{\prime \prime}$ ), this half power radius is dominated by the point spread function (PSF), and is likely to be over-estimated. The radii in physical units $(\mathrm{kpc})$ corresponding to $r_{\max }$ and $r_{\mathrm{hp}}$ are also given in Table 7.

The hard X-ray sources are compact and often point-like. We note however that there are some objects in which hard X-ray emission is resolved, e.g., Arp 220, and UGC 8387 (=IC 883).
As a guide, the PSF simulated at an energy of $3 \mathrm{keV}$ and normalized to the central peak of the hard X-ray emission is plotted along side the observed surface brightness profiles. In Table 7, the hard X-ray morphology is classified into three categories $\mathrm{R} / \mathrm{A} / \mathrm{P}$. When more than 2 data points deviate by more than $2 \sigma$ from the PSF it is denoted as R (resolved). When the data points agree with the PSF within the error bars, it is denoted as P (pointlike). Any other case is denoted as A (ambiguous).

Figure 9 shows a plot of $R_{\max }$ versus the soft X-ray luminosity. No clear correlation is seen even if objects containing AGN are removed.

The variety of shapes observed in the soft X-ray radial profiles could be related to the origin of the X-ray emission. Generally speaking, a gravitationally bound, virialized system shows an exponential profile while a power-law profile is expected from a free-flowing outflow from a compact central source. However, given the angular scale for our sample galaxies $\left(0.24-1.74 \mathrm{kpc} \mathrm{arcsec}^{-1}\right)$, any compact nuclear starbursts are likely to be contained within the innermost 1 " bin. There are several objects showing a power-law type, peaky, soft X-ray profile, and they are noted in the Notes on Individual Objects (Appendix A). Absorption can modify the soft X-ray profile if it has a radial dependence, e.g., centrally concentrated absorption, which suppress the brightness of the compact nuclear component, e.g. Arp 220 and VV 340 N.

\section{Discussion}

In Sect. 4.3, we used relatively crude spectral information based on the X-ray colour, (i.e. $H R$ ), to assess the presence of an AGN. Here we discuss further characterizations of the ACIS spectra for sufficiently bright objects. Before presenting the spectral analyses, a brief summary of the properties of X-ray sources in LIRGs and their spectra are described below.

The primary origin of the X-ray emission in LIRGs is considered to be a starburst and/or AGN. Given the dusty nature of LIRGs, if an AGN is present, it is likely to be an absorbed source. Such absorbed X-ray sources can be selected by X-ray colour analysis, as shown in Sect. 4.3. When the absorbing column density exceeds $10^{24} \mathrm{~cm}^{-2}$ and the absorbed transmitted component moves out of the Chandra bandpass, the identification of an AGN becomes difficult as it must rely on the detection of the Fe $\mathrm{K}$ line at $6.4 \mathrm{keV}$ in faint reflected emission (see the discussion in Sect. 4.3), and the data are often not of sufficient depth to make a clear detection. Such heavy nuclear obscuration could, however, occur in many LIRGs, and sometimes the hard $\mathrm{X}$-ray band is the only available window to check for signatures of an AGN, e.g. as for NGC 4945 (e.g., Iwasawa et al. 1993; Spoon et al. 2000).

In a starburst, there are various sources of X-ray emission (e.g., Persic \& Rephaeli 2002) whose origin can be traced back to massive stars. Individual supernovae and their remnants, stellar-wind heated ISM in star clusters, and X-ray binaries can all be X-ray sources. In particular, high-mass X-ray binaries (HMXBs) are considered to be the dominant source for the X-ray emission above $2 \mathrm{keV}$. A good correlation between the $2-10 \mathrm{keV}$ luminosity and the star formation rate have been found for nearby star forming galaxies (Ranalli et al. 2003; Grimm et al. 2003; Gilfanov et al. 2004) as well as for late type galaxies at higher redshift (Lehmer et al. 2008). The galacticscale emission nebulae, traced by $\mathrm{H} \alpha$ and the soft X-ray emission in local starburst galaxies like M 82, suggested that these extended nebulae are produced by the shock heated interstellar medium, swept up by a starburst-driven outflow (see e.g., 
A\&A 529, A106 (2011)

Table 7. Properties derived from the X-ray radial surface brightness profiles.

\begin{tabular}{|c|c|c|c|c|c|c|c|}
\hline No. & Galaxy & $\begin{array}{c}A S \\
\left(\mathrm{kpc} \operatorname{arcsec}^{-1}\right)\end{array}$ & $\begin{array}{c}r_{\max } \\
(\operatorname{arcsec})\end{array}$ & $\begin{array}{l}R_{\max } \\
(\mathrm{kpc})\end{array}$ & $\begin{array}{c}r_{\mathrm{hp}} \\
(\operatorname{arcsec})\end{array}$ & $\begin{array}{c}R_{\mathrm{hp}} \\
(\mathrm{kpc})\end{array}$ & $\begin{array}{c}\text { Morph } \\
H X\end{array}$ \\
\hline \multicolumn{8}{|c|}{ Cycle 8 Data } \\
\hline 1 & F01364-1042 & 0.93 & 6.5 & 6.04 & 2.0 & 1.86 & $\mathrm{P}$ \\
\hline 3 & II Zw 31 & 1.06 & 6.0 & 6.36 & 2.0 & 2.12 & $\mathrm{R}$ \\
\hline 5 & ESO 255-IG7 N & 0.76 & 7.0 & 5.32 & 2.5 & 1.90 & $\mathrm{R}$ \\
\hline 5 & ESO 255-IG7 C & 0.76 & 5.0 & 3.80 & 2.0 & 1.52 & A \\
\hline 5 & ESO 255-IG7 S & 0.76 & 7.0 & 5.32 & 2.5 & 1.90 & $\mathrm{R}$ \\
\hline 6 & 07251-0248 & 1.74 & 4.0 & 6.96 & 1.5 & 2.61 & - \\
\hline 7 & ESO 60-IG16 & 0.93 & 7.0 & 6.51 & 2.0 & 1.86 & $\mathrm{P}$ \\
\hline 9 & 09022-2615 & 1.19 & 5.5 & 6.54 & 1.5 & 1.78 & $\mathrm{R}$ \\
\hline 10 & F09111-1007 E & 1.08 & 4.0 & 4.32 & 1.0 & 1.08 & A \\
\hline 10 & F09111-1007 W & 1.08 & 4.0 & 4.32 & 1.0 & 1.08 & A \\
\hline 13 & ESO 374-IG32 & 0.73 & 8.0 & 5.84 & 2.5 & 1.82 & $\mathrm{R}$ \\
\hline 14 & F10173+0828 & 0.99 & - & - & - & - & A \\
\hline 19 & $13120-5453$ & 0.63 & 3.0 & 1.89 & 1.0 & 0.63 & $\mathrm{P}$ \\
\hline 20 & VV $250 \mathrm{E}$ & 0.63 & 5.5 & 3.46 & 1.5 & 0.95 & $\mathrm{R}$ \\
\hline 20 & VV $250 \mathrm{~W}$ & 0.63 & 6.0 & 3.78 & 2.5 & 1.57 & A \\
\hline 21 & UGC 8387 & 0.50 & 9.0 & 4.50 & 3.0 & 1.50 & $\mathrm{R}$ \\
\hline 24 & F14378-3651 & 1.37 & 3.0 & 4.11 & 1.0 & 1.37 & $\mathrm{P}$ \\
\hline 25 & VV $340 \mathrm{~N}$ & 0.70 & 18.0 & 12.60 & 5.5 & 3.85 & $\mathrm{R}$ \\
\hline 25 & VV $340 \mathrm{~S}$ & 0.70 & $>12.0$ & $>8.40$ & 7.5 & 5.25 & - \\
\hline 29 & ESO 69-IG6 (N) & 0.94 & 3.0 & 2.82 & 1.0 & 0.94 & $\mathrm{R}$ \\
\hline 31 & $\mathrm{~F} 17132+5313$ & 1.02 & 7.5 & 7.65 & 2.5 & 2.55 & $\mathrm{R}$ \\
\hline 33 & F18293-3413 & 0.38 & 10.0 & 3.80 & 3.0 & 1.14 & $\mathrm{R}$ \\
\hline 34 & ESO 593-IG8 & 0.98 & 12.0 & 11.76 & 4.5 & 4.41 & $\mathrm{R}$ \\
\hline 35 & F19297-0406 & 1.72 & 6.0 & 10.32 & 2.5 & 4.30 & $\mathrm{P}$ \\
\hline 36 & $19542+1110$ & 1.30 & 5.0 & 6.50 & 1.0 & 1.30 & $\mathrm{P}$ \\
\hline 37 & CGCG 448-020 & 0.72 & 10.0 & 7.20 & 3.0 & 2.16 & $\mathrm{R}$ \\
\hline 39 & $21101+5810$ & 0.77 & 6.0 & 4.62 & 1.5 & 1.16 & $\mathrm{P}$ \\
\hline 40 & ESO 239-IG2 & 0.85 & 6.0 & 5.10 & 1.5 & 1.27 & $\mathrm{P}$ \\
\hline 41 & F22491-1808 & 1.53 & 5.5 & 8.42 & 2.0 & 3.06 & $\mathrm{P}$ \\
\hline 43 & ESO 77-IG14 N & 0.84 & 4.0 & 3.36 & 1.0 & 0.84 & A \\
\hline 43 & ESO 77-IG14 S & 0.84 & 3.5 & 2.94 & 2.0 & 1.68 & $\mathrm{P}$ \\
\hline \multicolumn{8}{|c|}{ Archival Data } \\
\hline 4 & F05189-2524 & 0.83 & 2.0 & 1.7 & 1.0 & 0.83 & $\mathrm{P}$ \\
\hline 8 & F08572+3915 & 1.13 & - & - & - & - & A \\
\hline 11 & UGC $4881 \mathrm{E}$ & 0.79 & 2.5 & 2.0 & 1.0 & 0.79 & $\mathrm{P}$ \\
\hline 11 & UGC $4881 \mathrm{~W}$ & 0.79 & 8.0 & 7.0 & 3.5 & 3.1 & - \\
\hline 12 & UGC 5101 & 0.79 & 6.0 & 4.7 & 1.5 & 1.2 & $\mathrm{P}$ \\
\hline 15 & $F 10565+2448$ & 0.87 & 7.0 & 6.1 & 2.0 & 1.7 & $\mathrm{P}$ \\
\hline 16 & NGC 3690 E & 0.24 & $>13$ & $>3.1$ & - & - & $\mathrm{R}$ \\
\hline 16 & NGC $3690 \mathrm{~W}$ & 0.24 & $>13$ & $>3.1$ & - & - & $\mathrm{R}$ \\
\hline 17 & F12112+0305 & 1.42 & 8.0 & 11.3 & 4.0 & 5.7 & $\mathrm{P}$ \\
\hline 18 & UGC 8058 & 0.85 & 5.0 & 4.3 & 1.0 & 0.85 & $\mathrm{P}$ \\
\hline 22 & UGC 8696 & 0.77 & 9.0 & 6.9 & 2.5 & 1.9 & $\mathrm{R}$ \\
\hline 23 & F14348-1447 & 1.58 & 6.5 & 10.3 & 3.0 & 4.8 & $\mathrm{P}$ \\
\hline 26 & VV 705 & 0.82 & 4.5 & 3.7 & 1.5 & 1.2 & A \\
\hline 27 & $F 15250+3608$ & 1.10 & 4.0 & 4.4 & 1.5 & 1.6 & - \\
\hline 28 & UGC 9913 & 0.41 & 30 & 12.2 & 8.5 & 3.5 & $\mathrm{R}$ \\
\hline 30 & NGC 6240 & 0.53 & 15 & 8.0 & 5.5 & 2.9 & $\mathrm{R}$ \\
\hline 32 & F17207-0014 & 0.87 & 11.0 & 9.6 & 3.0 & 2.6 & $\mathrm{R}$ \\
\hline 38 & ESO 286-IG19 & 0.85 & 9.0 & 7.7 & 3.5 & 3.0 & $\mathrm{R}$ \\
\hline 42 & ESO 148-IG2 & 0.88 & 7.0 & 6.2 & 3.5 & 3.1 & $\mathrm{R}$ \\
\hline 44 & F23365+3604 & 1.22 & 3.0 & 3.7 & 1.0 & 1.2 & A \\
\hline
\end{tabular}

Notes. The radius where the soft X-ray brightness goes below $1 \%$ of the peak brightness at 1 " resolution is defined as $r_{\max }$ (arcsec). The corresponding physical radius, $R_{\max }(\mathrm{kpc})$, is also listed. The half power radius, $r_{\mathrm{hp}}(\operatorname{arcsec})$, is where the cumulative soft X-ray counts exceed half of the integrated counts within $r_{\max }$ at $1^{\prime \prime}$ resolution. The corresponding physical radius, $R_{\mathrm{hp}}(\mathrm{kpc})$, is also given. Whether the $2-7 \mathrm{keV}$ emission is resolved or not is indicated by "P" (point-like), "R" (resolved), and "A" (ambiguous).

Veilleux et al. 2005, for a review on galactic outflow phenomena; and Tomisaka \& Ikeuchi 1988; Suchkov et al. 1996; TenorioTagle \& Munoz-Yunon 1998; Strickland \& Stevens 2000, for simulations). As hypothesized by Chevallier \& Clegg (1985), hot $\left(T \sim 10^{8} \mathrm{~K}\right)$, high-pressure gas, produced via the collective kinetic energy from supernovae and massive stellar winds, is considered to drive the outflow. Given the temperature, this hot gas could be a hard X-ray source, but it is also expected to be 
rarefied, and thus not very radiative. The presence of weak emission from such gas has been suggested for M 82 (Griffiths et al. 2000; Strickland \& Heckman 2007; Ranalli et al. 2008) and NGC 253 (Pietsch et al. 2001).

\subsection{X-ray spectra and derived properties}

Except for some sources which are clearly absorbed AGN, the 0.4-7 keV Chandra spectra of the majority of the GOALS sources appear similar - namely an emission-line dominated soft $\mathrm{X}$-ray band (below $2 \mathrm{keV}$ ) and a hard X-ray tail. The relative composition of these two components is not widely different between objects, as the HR distribution (Fig. 5) shows. Objects that have a spectrum of this type always show totally different X-ray morphologies in the soft and hard X-ray bands (see the images included in the Appendix), suggesting distinct origins for hard and soft X-rays. Therefore, we will discuss the soft and hard band spectra separately below ${ }^{5}$.

As mentioned above, the hard X-ray emission is generally attributed to HMXBs, but this may not always be true for our objects. After excluding objects with clear AGN signatures, the hard band spectra of the remaining objects have relatively low signal to noise mainly due to the relative weakness of the source, hence they have been referred to (see Sect. 4.3) as HXQ galaxies in Iwasawa et al. (2009). Any spectral feature like an Fe K line is not evident in individual noisy spectra. However, the stacked spectrum of the 29 HXQ galaxies in our C-GOALS sample shows a strong high-ionization Fe $\mathrm{K}$ line (Fe xxv) at $6.7 \mathrm{keV}$ with $E W$ of $\simeq 0.9 \mathrm{keV}$ (Iwasawa et al. 2009). This means that a non negligible fraction of the HXQ galaxies have strong Fe Xxv emission in their hard band spectra, but they are buried in the noise in the individual spectra. Such a strong Fe xxv is not seen in the spectrum of HMXBs in our Galaxy, except when they are in an eclipse phase - they normally show a much weaker Fe line at $6.4 \mathrm{keV}$ (e.g. White et al. 1983; Torrejon et al. 2010). This means that, unlike for local star-froming galaxies, HMXBs are not the primary source of the hard X-ray band emission seen in the more luminous C-GOALS objects. Also the $2-10 \mathrm{keV}$ luminosity of the HXQ galaxies deviate from the extrapolated correlation line on the X-ray quiet side $(\sim 0.7$ dex on average, Iwasawa et al. 2009). Most objects used to derive the X-ray vs. FIR correlation (e.g., Ranalli et al. 2003) have $L_{\text {ir }}<10^{11} L_{\odot}$, while the lower bound of the IR luminosity of our sample is $10^{11.73} L_{\odot}$. This suggests that a transition in the nature of the dominant hard X-ray source in LIRGs occurs somewhere in the range $\log \left(L_{\mathrm{ir}} / L_{\odot}\right)=11.0-11.73$.

In the soft X-ray band, at the spectral resolution of a CCD spectrometer like the ACIS, heavy blending of emission lines makes it difficult to distinguish between photoionized gas irradiated by an AGN and thermal gas induced from a starburst. However, it is genrally assumed that thermal emission is responsible for the emission-line dominated spectra of LIRGs in the soft X-ray band, and this appears even to be true for an object

\footnotetext{
5 It is generally possible to reproduce a Chandra full-band spectrum for those objects whose spectra can be represented as the sum of a power-law and a thermal emission spectrum with sub-keV temperature and solar abundance. A good fit can often be found with the thermal spectrum accounting only for the emission-line bump around $1 \mathrm{keV}$, leaving not only the hard band $(\geq 2 \mathrm{keV})$ emission but also the softest emission below $\sim 0.7 \mathrm{keV}$ to the power-law. This spectral decomposition would be invalid when the imaging data show a totally different spatial distribution between these two bands (as seen in our sample objects), because they cannot come from the same component represented by the power-law (see Appendix B).
}

Table 8. X-ray spectral slopes.

\begin{tabular}{rlcc}
\hline \hline No. & \multicolumn{1}{l}{ Galaxy } & \multicolumn{1}{c}{$\Gamma_{\mathrm{S}}$} & $\Gamma_{\mathrm{H}}$ \\
\hline \multicolumn{4}{c}{ Cycle 8 targets } \\
3 & VII Zw 31 & $2.7_{-0.4}^{+0.4}$ & - \\
5 & ESO 255-IG7 N & $2.0_{-0.6}^{+0.7}$ & - \\
5 & ESO 255-IG7 C & $2.0_{-0.4}^{+0.5}$ & - \\
7 & ESO 60-IG16 & $2.1_{-1.3}^{+1.4}$ & $0.7_{-0.8}^{+0.3}$ \\
9 & 09022-3615 & $2.3_{-0.6}^{+0.6}$ & $-0.1_{-0.3}^{+0.8}$ \\
10 & F09111-1007 E & $3.1_{-0.8}^{+0.9}$ & - \\
19 & 13120-5453 & $1.0_{-0.4}^{+0.4}$ & $2.6_{-0.9}^{+1.5}$ \\
20 & VV 250 E & $1.7_{-0.4}^{+0.4}$ & $3.6_{-0.8}^{+0.9}$ \\
21 & UGC 8387 & $2.5_{-0.4}^{+0.4}$ & - \\
25 & VV 340 N & $3.8_{-0.4}^{+0.4}$ & $-2.5_{-0.5}^{+1.7}$ \\
29 & ESO 69-IG6 N & $3.0_{-0.4}^{+0.4}$ & - \\
31 & F17132+5313 & $2.4_{-1.1}^{+1.4}$ & - \\
33 & F18293-3413 & $2.7_{-0.3}^{+0.4}$ & $2.0_{-1.0}^{+0.4}$ \\
34 & ESO 593-IG8 & $2.2_{-0.6}^{+0.6}$ & - \\
35 & F19297-0406 & $3.6_{-1.4}^{+1.5}$ & - \\
36 & 19542+1110 & $\sim-2.8$ & $1.3_{-0.5}^{+0.2}$ \\
37 & CGCG 448-020 & $2.3_{-0.3}^{+0.3}$ & $1.7_{-0.4}^{+0.5}$ \\
40 & ESO 239-IG2 & $2.7_{-0.4}^{+0.4}$ & - \\
& & & \\
4 & Archival data & \\
4 & F05189-2524 & $\sim 0$ & $0.48_{-0.08}^{+0.20}$ \\
12 & UGC 5101 & $2.0_{-0.3}^{+0.3}$ & $\sim-0.7$ \\
15 & F10565+2448 & $2.5_{-0.3}^{+0.3}$ & - \\
16 & NGC 3690 E & $1.9_{-0.2}^{+0.2}$ & $1.0_{-0.7}^{+0.7}$ \\
16 & NGC 3690 W & $1.9_{-0.2}^{+0.2}$ & $1.1_{-0.5}^{+0.5}$ \\
18 & UGC 8058 core & $1.9_{-0.1}^{+0.2}$ & $0.71_{-0.06}^{+0.15}$ \\
18 & UGC 8058 nebula & $5.2_{-0.3}^{+0.3}$ & - \\
22 & UGC 8696 & $2.8_{-0.2}^{+0.2}$ & $-1.8_{-0.3}^{+0.1}$ \\
26 & VV 705 N & $2.4_{-0.5}^{+0.6}$ & - \\
28 & UGC 9913 & $1.8_{-0.2}^{+0.2}$ & $0.8_{-0.3}^{+0.5}$ \\
32 & F17207-0014 & $2.0_{-0.3}^{+0.3}$ & $0.7_{-0.4}^{+0.9}$ \\
38 & ESO 286-IG19 & $3.8_{-0.2}^{+0.2}$ & $0.7_{-2.0}^{+1.0}$ \\
42 & ESO 148-IG2 N & $3.0_{-0.4}^{+0.5}$ & - \\
\hline & & $1.3_{-0.3}^{+0.3}$ & $1.4_{-0.4}^{+0.3}$ \\
\hline
\end{tabular}

Notes. Photon indices derived by fitting a power-law to the $0.8-2 \mathrm{keV}$ $\left(\Gamma_{\mathrm{S}}\right)$ and 3-7 keV $\left(\Gamma_{\mathrm{H}}\right)$ bands after correcting for Galactic absorption. Measurements are given only when the detected source counts exceed $50 \mathrm{cts}$ in the respective bands in order for the spectral slope to have meaningful constraints.

like NGC 6240 in which AGN signatures appear only in the hard band (Netzer et al. 2005). Thus we also utilize the thermal emission spectrum to compare with the soft X-ray spectra. However, we still suspect that there might be a possible contribution from photoionized gas to the soft X-ray spectra of some objects, as will be discussed below.

In applying a thermal emission model, one problem specific to our objects is the assumed solar abundance. The hot gas induced by a starburst is expected to be polluted heavily by ejecta of core collapse supernovae, which is rich in $\alpha$ elements relative to iron. Therefore the abundance pattern should deviate significantly from the solar pattern, and this has been found to be the case for starburst knots in nearby galaxies, e.g., The Antennae (Fabbiano et al. 2004). When the data quality is good, a standard 
thermal emission spectrum with a solar abundance pattern as computed by, e.g., MEKAL, indeed does not agree with some of the observed emission line strengths, and a modified abundance pattern, rich in $\alpha$ elements, gives a better description. This may not be evident for poor quality spectra. Since the data quality between our spectra varies, we first measure a spectral slope and then fit the thermal emission model with the abundance pattern of core collapse SNe for all of the soft X-ray data. These spectral fits were performed to the count rate spectra by comparing the model folded through the detector response, using XSPEC (ver. 11).

\subsubsection{Spectral slopes}

As a first order characterization of the spectral shape, spectral slopes in the soft $(0.8-2 \mathrm{keV})$ and hard (3-7 keV) bands, derived from power-law fitting are given in Table 8 . The photon index, $\Gamma$, is the slope parameter of a power-law model for describing a photon spectrum, defined as $\mathrm{d} N / \mathrm{d} E \propto E^{-\Gamma}$ photons $\mathrm{cm}^{-2} \mathrm{~s}^{-1} \mathrm{keV}^{-1}$, and is related to the energy index $\alpha\left(F_{\mathrm{E}} \propto\right.$ $\left.E^{-\alpha}\right)$ with $\Gamma=\alpha+1$. In order to obtain meaningful constraints, the slopes were determined for those spectral data which have more than $50 \mathrm{cts}$ in the energy range of interest. In fitting the power-law, Galactic absorption is assumed.

In most objects, the soft X-ray emission is dominated by (largely unresolved) emission lines, and the spectrum turns over below $0.8 \mathrm{keV}$ where the Fe L complex becomes weak. The power-law fits for the soft X-ray spectra are therefore mainly for the emission-line blend, not the underlying continuum.

\subsubsection{Prominent emission lines}

The Fe $\mathrm{K}$ line is a well known diagnostic of heavily obscured AGN, and it is clearly seen in some spectra of our sample galaxies. Another prominent spectral line we observe is $\mathrm{Si} \mathrm{K}$ at $\sim 1.8 \mathrm{keV}$. The strongest line is usually from Si xIII at $1.85 \mathrm{keV}$. This line is relatively isolated in the soft X-ray range so that the line properties are easier to measure than is the case for other emission lines. It could also provide a clue to the origin of the $\mathrm{X}$-ray emission.

When a line is detected at $>2 \sigma$ after fitting with a Gaussian, its line flux and equivalent width with respect to the neighboring continuum are measured. Tables 9 and 10 lists measured values for the $\mathrm{Si}$ and $\mathrm{Fe}$ lines, respectively. Fe $\mathrm{K}$ features are found at $6.4 \mathrm{keV}$ (line emission from cold $\mathrm{Fe}$ ) and/or at higher energies (Fe xxv or Fe xxvI). The detection of these Fe K lines have been reported previously, based on XMM-Newton, ASCA, and Chandra observations (Imanishi et al. 2003; Xia et al. 2002; Armus et al. 2009; Komossa et al. 2003; Della ceca et al. 2002; Ballo et al. 2004; Clements et al. 2002; Iwasawa et al. 2005; Iwasawa \& Comastri 1998; Turner \& Kraemer 2003; Braito et al. 2004). One addition is F17207-0014 for which a possible highionization $\mathrm{Fe} \mathrm{K}$ line is detected $(2 \sigma)$ for the first time.

\subsubsection{Metal abundance pattern}

As discussed above, the soft X-ray spectrum may be more complicated than a standard thermal emission spectrum with a solar metal-abundance pattern. For a more physically motivated characterization of the soft X-ray emission than given by a simple power-law fit, we adopt a thermal emission model with a Type II SN abundance pattern (e.g., Nomoto et al. 1997) to fit the $0.4-2 \mathrm{keV}$ spectra. The nominal metallicity ratio relative to oxygen for various metals is assumed (as given in Dupke \& Arnaud 2001) and varies together in the fit: $(\mathrm{Mg}, \mathrm{Si}) / \mathrm{O}=1,(\mathrm{Ne}, \mathrm{S}) / \mathrm{O}$ $=0.67,(\mathrm{Ar}, \mathrm{Ca}, \mathrm{Ni}) / \mathrm{O}=0.46, \mathrm{Fe} / \mathrm{O}=0.27$. We note that this

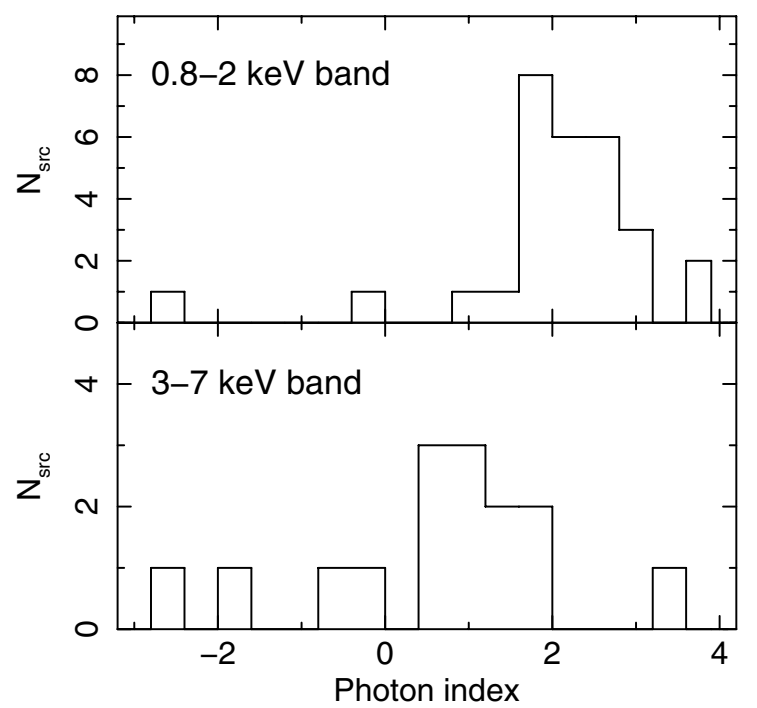

Fig. 10. Distribution of spectral slope (photon index) measured by a power-law fit in the $0.8-2 \mathrm{keV}$ and $3-7 \mathrm{keV}$ bands. A correction for Galactic absorption has been made.

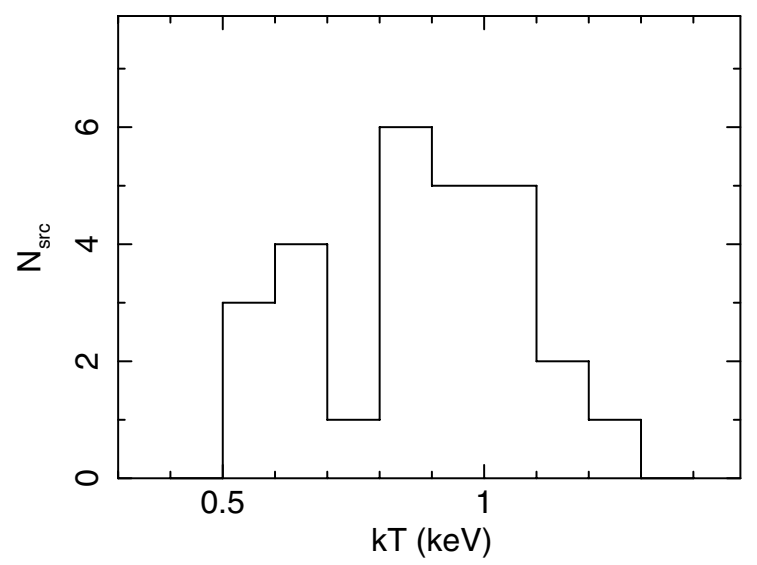

Fig. 11. The distribution of temperature, $k T$, when the thermal emission spectrum is fitted to the $0.4-2 \mathrm{keV}$ data (see Table 11). The median value is $k T=0.88 \mathrm{keV}$.

abandance pattern is one of those calculated for core-collapse $\mathrm{SNe}$ and may not be very accurate, but it deviates significantly from the solar pattern and will provide a good reference against the observed data. The metallicity measurement is primarily driven by the strength of the Fe L complex around $1 \mathrm{keV}$ at the temperature assumed for the gas. The metallicity results are given for the $\mathrm{O}$ abundance relative to solar $\left(Z_{\mathrm{SNII}}\right.$ in Table 11) in the interest of $\alpha$ elements, i.e., $Z_{\mathrm{SNII}}$ is an "equivalent" oxygen abundance, which is mainly determined by the Fe $\mathrm{L}$ feature and converted by the above $\mathrm{Fe} / \mathrm{O}$ ratio, since given the quality of the data, the oxygen line (e.g., O vIII at $0.65 \mathrm{keV}$ ) itself cannot give a strong constraint. Here the solar abundance is that of Anders \& Grevess (1989). Emission lines of He-like Mg and Si are located at energies where the ACIS response is good, and in some qood quality data their metallicity can be measured independently (when they are strong). When this is the case, their metallicity measurements are given separately. In this case, Si and $\mathrm{Mg}$ are assumed to have the same abundance.

This prescription described above usually gives better agreement with the data than that achieved with a solar abundance pattern, as demonstrated for the spectrum of ESO 286-IG19 in Fig. 12. When a solar abundance pattern is assumed, the 

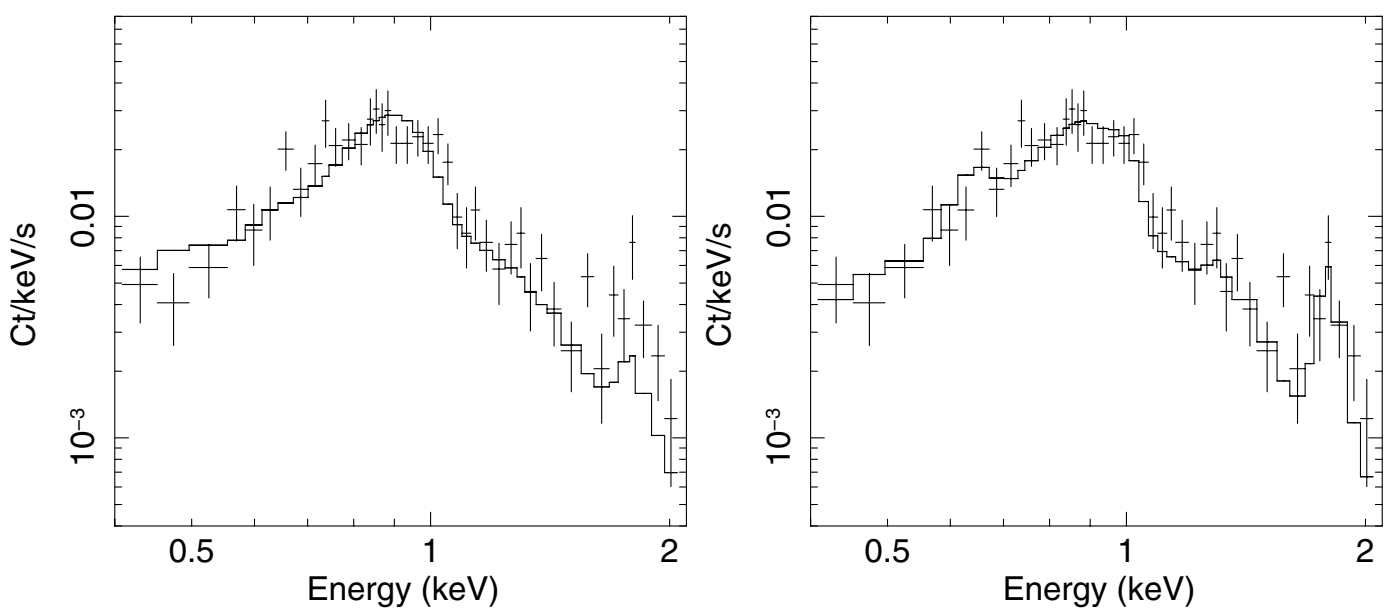

Fig. 12. The Chandra ACIS 0.4-2 keV spectrum of ESO 286-IG19, fitted with a thermal emission spectrum (MEKAL) with a solar abundance pattern (left) and that of the core-collapse SNe (right), in which O viII $(0.65 \mathrm{keV}), \mathrm{Ne}$ Ix $(1.0 \mathrm{keV}), \mathrm{Mg} \mathrm{XI}(1.36 \mathrm{keV})$ and $\mathrm{Si}$ xIII $(1.85 \mathrm{keV})$ are more pronounced. The $\chi^{2}$ values for the two fits are 53 and 37 for 41 and 40 degrees of freedom, respectively.

metallicity is found to be only $0.21_{-0.03}^{+0.05} Z_{\odot}$, and clearly underestimates emission-lines such as Si xIII, which would be better described with $\sim 2 Z_{\odot}$ (Table 11). The temperature measurement is also affected by the choice of abundance pattern. In the example of ESO 286-IG19, the temperature derived from the solar abundance pattern is $k T=0.82 \pm 0.03$, while the SNII pattern gives $k T=0.70 \pm 0.04 \mathrm{keV}$.

The bright X-ray core of Mrk 231 is dominated by the unresolved nuclear component even in the soft X-ray band. Given that a single thermal emission model is not able to give a reasonable fit, the spectral parameters are not presented in Table 11.

The argument for a non-solar abundance pattern has so far been based on a single-temperature fit. One caveat is that a multitemperature model could mimic the same effect (e.g., Buote \& Fabian 1998) in some cases, since it is difficult to distinguish between the two interpretations with spectral data at the CCD resolution of the ACIS. High-resolution spectroscopy that could identify individual lines could be used to determine the characteristic temperature of the gas. This would tell whether the gas is in a multi-phase. At least for the very extended nebulae, as seen in Mrk 231 and Mrk 273, which emit almost no emission above $2 \mathrm{keV}$ but strong Mg XI and Si XIII, higher temperature gas is unlikely to be present. We note $\alpha$-element rich gas would be produced naturally if a starburst is responsible for the metal enrichment.

\subsubsection{Strong Si XIII emission lines and their origin}

Even when assuming the $\alpha$-element rich abundance pattern of core-collapse $\mathrm{SNe}$, the observed Si metallicity sometimes requires an even larger value (see Table 11). This may simply be due to more $\mathrm{Si}$ enriched gas than theoretically predicted. However, the excess Si line intensity could also be due to an additional source of the line emission, e.g., photoionized gas illuminated by a hidden AGN. In fact, among the objects listed in Table 11, all of the AGN that are identified by spectral hardness and/or the Fe K diagnostics show at least an excess of $\times 2$ in the $\mathrm{Si}$ metallicity relative to $Z_{\mathrm{SNII}}$. Since the energies around Si XIII $(1.84 \mathrm{keV})$ are where the spectrum of the soft X-ray emitting thermal gas starts to decline rapidly, an extra contribution from any AGN related component would stand out relatively well.

Here, we examine the Si line strengths of starbursts and AGN in an alternative way by using the Si XIII detected objects in
Table 9. Si XIII (1.84 keV) measurements.

\begin{tabular}{llccc}
\hline \hline No. & Galaxy & $\begin{array}{c}I_{\mathrm{Si}} \\
10^{-6} \mathrm{ph} \mathrm{cm}^{-2} \mathrm{~s}^{-1}\end{array}$ & $\begin{array}{c}E W_{\mathrm{Si}} \\
\mathrm{keV}\end{array}$ & Note \\
\hline 10 & F09111-1007 E & $0.74_{-3.7}^{+4.5}$ & 0.44 & \\
12 & UGC 5101 & $0.66_{-0.30}^{+0.22}$ & 0.23 & \\
15 & F10565+2448 & $6.1_{-2.8}^{+3.4}$ & 0.17 & \\
16 & NGC 3690 E & $2.3_{-1.1}^{+1.1}$ & 0.15 & \\
18 & UGC 8058 core & $0.60_{-0.27}^{+0.26}$ & 0.05 & \\
18 & UGC 8058 nebula & $0.30_{-0.14}^{+0.15}$ & 0.39 & \\
19 & 13120-5453 & $2.1_{-0.8}^{+0.8}$ & 0.20 & \\
21 & UGC 8387 & $1.5_{-0.7}^{+0.7}$ & 0.26 & \\
25 & VV 340 N & $1.1_{-0.5}^{+0.4}$ & 0.26 & \\
28 & UGC 9913 & $1.3_{-0.5}^{+1.5}$ & 0.23 & $a$ \\
29 & ESO 69-IG6 N & $1.3_{-0.6}^{+0.6}$ & 0.34 & \\
30 & NGC 6240 & $20.4_{-4.0}^{+3.5}$ & 0.30 & $a$ \\
32 & F17207-0014 & $0.50_{-0.20}^{+0.42}$ & 0.14 & \\
33 & F18293-3413 & $4.0_{-1.0}^{+0.8}$ & 0.42 & \\
34 & ESO 593-IG8 & $0.85_{-0.43}^{+0.54}$ & 0.26 & \\
37 & CGCG 448-020 & $1.1_{-0.5}^{+0.7}$ & 0.16 & $b$ \\
38 & ESO 286-IG19 & $0.59_{-0.26}^{+0.28}$ & 0.16 & \\
\hline
\end{tabular}

Notes. Detections with $2 \sigma$ or higher significance are listed. ${ }^{(a)}$ This line intensity is of a blend of Si XIII and Si xIV and is measured by fitting a broad Gaussian. ${ }^{(b)}$ This emission-line is not found at the expected energy of Si XIII, but at $1.60 \pm 0.06 \mathrm{keV}$.

Table 9. Since the metallicity, $Z_{\mathrm{SNII}}$, is driven by the Fe L emission (Sect. 5.1.3), the enhancement of $Z_{\mathrm{Si}}$ is basically the relative strength of the Si XIII line to the Fe L bump around $0.8-1 \mathrm{keV}$. As the heavily blended Fe L emission is not resolved at the CCD spectral resolution, the mean $0.8-1 \mathrm{keV}$ intensity is used as a crude measure of $\mathrm{Fe} \mathrm{L}$ emission, assuming that $\mathrm{Fe} \mathrm{L}$ dominates in this band. Thus, the mean $\mathrm{Si}$ XIII/Fe L ratio can be assessed between starburst and AGN.

The starburst sample consists of 7 objects, F10565+2448, NGC 3690 E, Arp 220, F17207-0014, F18293-3413, CGCG 448-020 and ESO 286-IG19. Note that objects with mid-IR [Nev] detection are not included (see below). The AGN sample consists of 5 known Compton-thick AGN, UGC 5101, Mrk 231, 13120-5453, VV 340 N and NGC 6240. 
Table 10. Fe K line features.

\begin{tabular}{rlccc}
\hline \hline No. & Galaxy & $\begin{array}{c}E \\
\mathrm{keV}\end{array}$ & $\begin{array}{c}I_{\mathrm{FeK}} \\
10^{-6} \mathrm{ph} \mathrm{s}^{-1} \mathrm{~cm}^{-2}\end{array}$ & $\begin{array}{c}E W_{\mathrm{FeK}} \\
\mathrm{keV}\end{array}$ \\
\hline \multicolumn{5}{c}{ Cold line } \\
4 & F05189-2524 & 6.4 & $6.0_{-2.8}^{+4.0}$ & 0.12 \\
12 & UGC 5101 & 6.4 & $0.68_{-0.31}^{+0.55}$ & 0.30 \\
18 & UGC 8058 & 6.4 & $1.0_{-0.4}^{+0.3}$ & 0.10 \\
22 & UGC 8696 & 6.4 & $6.7_{-2.4}^{+2.7}$ & 0.24 \\
25 & VV 340 N & 6.4 & $1.8_{-0.9}^{+1.1}$ & 1.2 \\
30 & NGC 6240 & 6.4 & $15.4_{2.0}^{+2.0}$ & 0.40 \\
\multicolumn{5}{c}{ High-ionzation line } \\
16 & NGC 3690 E & $6.65_{-0.02}^{+0.03}$ & $7.4_{-2.5}^{+2.1}$ & - \\
28 & UGC 9913 & $6.64_{-0.03}^{+0.03}$ & $0.92_{-0.32}^{+0.51}$ & 0.93 \\
30 & NGC 6240 & $6.65_{-0.02}^{+0.02}$ & $8.0_{-1.6}^{+2.0}$ & 0.16 \\
32 & F17207-0014 & $6.90_{-0.05}^{+0.05}$ & $0.82_{-0.41}^{+0.73}$ & 0.82 \\
\hline
\end{tabular}

Notes. Line detections with $2 \sigma$ or higher significance are listed. The cold $\mathrm{Fe} \mathrm{K} \alpha$ at $6.4 \mathrm{keV}$ and the high-ionization $\mathrm{Fe} \mathrm{K} \alpha$, Fe xxv or Fe xxvi are listed separately. The line centroid energy is measured in the rest frame in $\mathrm{keV}$.

When making a mean spectrum of each sample, a redshift correction was made due to the fact that the energy-scale shifts between objects are not negligible. The spectral data of individual objects are chosen so that the rest-frame $0.50-2.18 \mathrm{keV}$ range is covered and then they are binned at (rest-frame) $21 \mathrm{eV}$ intervals. These spectra are corrected for the effective area in the same way as those spectra in Fig. 4 (Sect. 4.2), which is necessary when the redshift correction for the energy scale is made. Since the soft X-ray band is sensitive even to variations in Galactic column $\left(N_{\mathrm{H}}\right.$ ranges between $9 \times 10^{19} \mathrm{~cm}^{-2}$ and $2.1 \times 10^{21} \mathrm{~cm}^{-2}$ for the relevant objects here), an absorption correction for the Galactic column is also made. Individual spectra are then normalised to the $0.8-1 \mathrm{keV}$ intensity before computing an average from the individual members of each sample.

The mean 0.5-2.2 keV spectra of the starburst and AGN samples are shown in Figs. 13a and b, where the 0.8$1 \mathrm{keV}$ intensity of both spectra has been set to the same level. The Si line strength relative to the Fe L emission can be readily compared. The Si line of the mean AGN spectrum appears to be stronger than that of the starburst by a factor of $2.0 \pm 0.6$. Albeit that this is a relatively crude measure, the result is consistent with an enhanced Si line in AGN spectra, as suggested by the metallicity fitting (Table 11).

Furthermore, an additional mean spectrum was constructed using the same procedure, for five objects which are not selected as AGN by the X-ray criteria, but which show a detectable mid-IR [Nev] line in their Spitzer IRS spectra (Petric et al. 2011): VII Zw 31, F09111-1007, UGC 8387, F17132+5313 and ESO 593-IG8 (see Table 5). The mean spectrum of these Ne v detected objects also suggests an enhancement of Si XIII; although the data are still noisy, the enhancement relative to the starburst sample is a factor of $1.8 \pm 0.9$.

While it is premature to propose such enhanced Si XIII as an alternative to the $\mathrm{Fe} \mathrm{K}$ diagnostic for a hidden AGN, it is interesting to note that both X-ray selected Compton thick AGN and mid-IR selected AGN candidates appear to show strong Si XIII on average, even when the data quality is not good in the $\mathrm{Fe} \mathrm{K}$ band. A contribution from an AGN photoionized component in addition to a thermal component can be a feasible explanation, unless the photoionized gas region is affected by internal absorption.
Table 11. Temperature, absorption and metallicity.

\begin{tabular}{|c|c|c|c|c|c|}
\hline No. & Galaxy & $\begin{array}{l}k T \\
\mathrm{keV}\end{array}$ & $\begin{array}{c}N_{\mathrm{H}} \\
10^{21} \mathrm{~cm}^{-2}\end{array}$ & $\begin{array}{c}Z_{\mathrm{SNII}} \\
Z_{\odot}\end{array}$ & $\begin{array}{l}Z_{\mathrm{Si}} \\
Z_{\odot}\end{array}$ \\
\hline \multicolumn{6}{|c|}{ Cycle 8 targets } \\
\hline 3 & VII Zw 31 & $1.0_{-0.1}^{+0.1}$ & - & $4_{-2}$ & $8_{-5}^{+20}$ \\
\hline 5 & ESO 255-IG7 N & $1.8_{-0.0}^{+0.8}$ & - & $0.4_{-04}^{+1.6}$ & - \\
\hline 9 & 09022-3615 & $1.1_{-0.2}^{+0.5}$ & - & $0.05_{-0.05}^{+0.4}$ & $0.4_{-0.2}^{+0.2}$ \\
\hline 10 & F09111-1007 E & $0.90_{-0.13}^{+0.18}$ & - & $1.0_{-0.5}^{+1.05}$ & $\begin{array}{l}-0.2 \\
4_{-2}^{+7}\end{array}$ \\
\hline 13 & ESO 374-IG32 & $0.68_{-012}^{+0.15}$ & - & $0.6_{-04}^{+3}$ & $1.7_{-12}^{+3.7}$ \\
\hline 19 & $13120-5453$ & $0.82^{+0.26}$ & - & $0.7^{+0.8}$ & - \\
\hline 20 & VV 250 & $1.0_{-0.1}^{+0.1}$ & - & $0.7_{-0.2}^{+0.5}$ & $2.6_{-0.7}^{+1.9}$ \\
\hline 21 & UGC 8387 & $1.0_{-01}^{+0.1}$ & 一 & $2.2_{-0.3}^{+2.3}$ & $7_{-3}^{+9.1}$ \\
\hline 25 & VV $340 \mathrm{~N}$ & $0.83_{-0.07}^{+0.08}$ & - & $1.3_{-04}^{+0.8}$ & $3.0_{-11}^{+2.6}$ \\
\hline 29 & ESO 69-IG6 N & $0.88_{-0.10}^{+0.10}$ & - & $4.5_{-1.9}^{+20}$ & $10_{-6}^{+18}$ \\
\hline 31 & $F 17132+5313$ & $0.59_{-0.10}^{+0.10}$ & - & $0.7_{-0.4}^{-1.9}$ & ${ }^{-0}$ \\
\hline 33 & F18293-3413 & $0.61_{-0.07}^{+0.08}$ & $7.2_{-1.0}^{+0.8}$ & $1.4_{-07}^{+4.9}$ & - \\
\hline 34 & ESO 593-IG8 & $0.95_{-0.11}^{+0.16}$ & - & $0.53_{-0.29}^{+0.62}$ & $2.1_{10}^{+1.5}$ \\
\hline 35 & F19297-0406 & $1.1_{-0.2}^{+0.3}$ & - & $5_{-4}^{-0.29}$ & - \\
\hline 37 & CGCG 448-020 & $0.66_{-0.09}^{+0.23}$ & $2.9_{-1.0}^{+0.7}$ & $0.09_{-0.09}^{+2.3}$ & - \\
\hline 40 & ESO 239-IG2 & $0.76_{-0.06}^{+0.07}$ & - & $1.2_{-0.6}^{+5}$ & $3.5_{-1.8}^{+10}$ \\
\hline \multicolumn{6}{|c|}{ Archival data } \\
\hline 12 & UGC 5101 & $0.89_{-0.07}^{+0.08}$ & - & $0.9_{-0.2}^{+0.2}$ & $4.7_{-14}^{+1.5}$ \\
\hline 15 & $F 10565+2448$ & $1.0_{-0.1}^{+0.1}$ & - & $1.1_{-0.5}^{+0.2}$ & $3.0_{-10}^{+1.6}$ \\
\hline 16 & NGC 3690 & $0.66_{-0.04}^{+0.104}$ & $2.4_{-0.3}^{+0.3}$ & $0.30_{-0.05}^{+0.05}$ & $0.43_{-0.10}^{-10.10}$ \\
\hline 18 & UGC 8058 neb & $0.50_{-0.03}^{+0.04}$ & -0.3 & $0.31_{-0.06}^{-0.05}$ & $0.96_{-0.33}^{+0.40}$ \\
\hline 22 & UGC 8696 & $0.88_{-0.04}^{+0.03}$ & - & $0.52_{-0.07}^{+0.08}$ & $1.1_{0.2}^{+0.23}$ \\
\hline 22 & UGC 8696 neb & $0.58_{-0.04}^{-0.04}$ & - & $1.3_{-0.3}^{+0.07}$ & $1.7_{0.8}^{+2.2}$ \\
\hline 23 & F14348-1447 & $0.92_{-0.15}^{-0.04}$ & - & $1.2_{-0.8}^{+0.30}$ & - \\
\hline 26 & VV $705 \mathrm{~N}$ & $0.94_{-0.09}^{-0.13}$ & - & $2.8_{-17}^{+3.8}$ & $8_{-4}^{+9}$ \\
\hline 28 & UGC 9913 & $0.90_{-0.06}^{+0.07}$ & $2.9_{-0.3}^{+0.4}$ & $0.37_{-0.10}^{+0.12}$ & $0.70_{-0.21}^{+0.28}$ \\
\hline 30 & NGC 6240 & $0.91_{-0.03}^{+0.06}$ & 3. $0_{-0 . x}^{-0.3}$ & $0.24_{-0.03}^{+0.10}$ & $0.54_{-0.05}^{+0.01}$ \\
\hline 32 & F17207-0014 & $0.59_{-0.10}^{+0.03}$ & $5.2_{-1.0}^{+1.0}$ & $0.30_{-0.15}^{+0.03}$ & $0.59_{-0.23}^{+0.025}$ \\
\hline 38 & ESO 286-IG19 & $0.70_{-0.04}^{+0.04}$ & - & $1.0_{-0.3}^{+0.3}$ & $2.0_{-0.5}^{+0.6}$ \\
\hline 42 & ESO $148-\mathrm{IG} 2 \mathrm{~N}$ & $0.84_{-0.10}^{-0.04}$ & - & $2.7_{-13}^{-10.3}$ & $6_{-3}^{-8.5}$ \\
\hline 42 & ESO 148-IG2 S & $1.01_{-0.18}^{+0.18}$ & - & $0.73_{-0.27}^{+0.42}$ & $3.2_{-1.0}^{+1.8}$ \\
\hline
\end{tabular}

Notes. The $0.4-2 \mathrm{keV}$ data are fitted with a thermal emission spectrum assuming the metal abundance pattern of core-collapse supernovae (see text for details). When excess absorption above the Galactic value is required, the best-fit column density $\left(N_{\mathrm{H}}\right)$ is listed. $Z_{\mathrm{SNII}}$ is the metallicity for oxygen, which is tied to the other elements with the assumed abundance pattern of Type II SNe. This value is mainly driven by the Fe metallicity, which is assumed to be 0.27 times that of the oxygen metallicity, and which fits the Fe L hump around $1 \mathrm{keV}$. When excess metallicity for $\mathrm{Si}$ (and $\mathrm{Mg}$ ) is required, the Silicon metallicity is fitted independently $\left(Z_{\mathrm{Si}}\right)$.

\subsection{Discrete $X$-ray emission from star clusters}

There are a few objects that show discrete X-ray sources separate from the main body of the galaxy emission. Some of these discrete X-ray sources have clear optical counterparts identified in our high resolution HST-ACS images. The optical counterparts are star clusters or dwarf galaxies in the tidal tails. In most cases, only a few X-ray counts are detected in the soft band. Inferred X-ray luminosities are on the order of $10^{39} \mathrm{erg} \mathrm{s}^{-1}$. They are much more powerful than the super star clusters in our Galaxy, for which the primary X-ray production mechanism is considered to be shock heating by stellar winds. If the X-ray emission in the C-GOALS objects comes from a single source in a star cluster, then this could be an example of an ultra-luminous X-ray source (ULX). Prime examples are the southern source in 


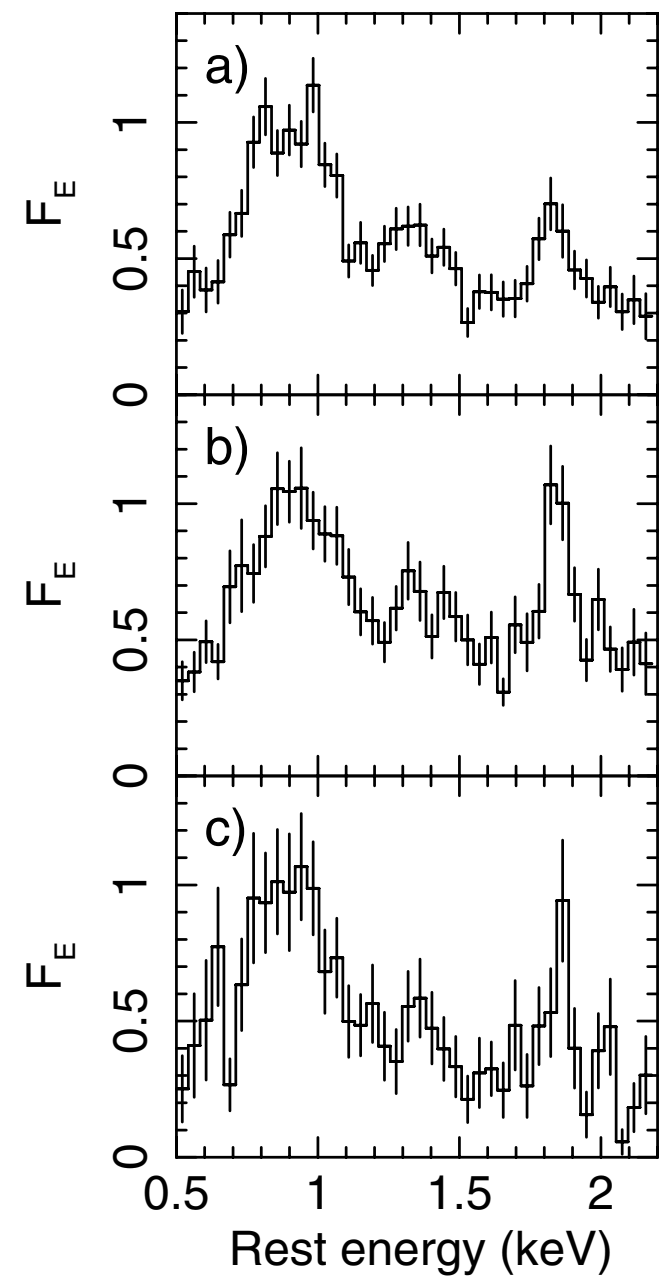

Fig. 13. The mean $0.5-2.2 \mathrm{keV}$ spectra of a) seven starbursts; b) five Compton-thick AGN; and c) five non X-ray AGN but with $[\mathrm{Nev}] \lambda 14.3 \mu \mathrm{m}$ detection. The energy scale is in the rest frame. The individual spectra have been corrected for Galactic absorption and the detector effective area and then normalized to the $0.8-1 \mathrm{keV}$ intensity before averaging within each sample. These mean spectra are constructed in order to facilitate comparison of the strength of Si XIII at $1.84 \mathrm{keV}$ relative to the $0.8-1 \mathrm{keV}$ band where $\mathrm{Fe} \mathrm{L}$ emission dominates between the different classes of objects (see text for detail).

UGC 8387, and the eastern triple source in CGCG 448-020 (see Fig. 2).

\section{Summary}

1. We present Chanda-ACIS data for a complete sample of 44 objects which represent the high luminosity portion of the GOALS sample. The objects in this C-GOALS sample have $\log \left(L_{\text {ir }} / L_{\odot}\right)=11.73-12.57$ with $z=0.010-0.088$, and represent the most luminous IR selected galaxies in the local universe. X-rays are detected from 43 out of the 44 objects, and their arcsec-resolution images, spectra, and radial brightness profiles are presented.

2. Objects with a clear AGN X-ray signature represent $37 \%$ $(16 / 44)$ of the total sample, and $75 \%(12 / 16)$ of these AGN are found in the higher IR luminosity half of the sample at $\left.\log \left(L_{\mathrm{ir}} / L_{\odot}\right)>12.0\right)$. The AGN fraction would increase to $48 \%$ if objects with [Ne v] $\lambda 14.3 \mu$ m detection are included. These AGN, however, appear to be a relatively minor $(\sim 10 \%)$ component in the total energy output of the host galaxies apart from a few exceptions.
3. While most objects show evidence for strong interactions/mergers, NGC 6240 remains the only object to clearly show a double AGN in our X-ray spectra. Eight AGN are found in single nucleus objects and an additional seven have $\mathrm{X}$-ray AGN in one of the double nuclei.

4. For objects without obvious X-ray AGN signatures, X-ray luminosities are found to be lower than expeted from their IR luminosities based on the correlation found for nearby star-forming galaxies with lower star formation rates. The hard X-ray emission of these objects does not always appear to be due to HMXBs, given that the stacked spectrum shows strong Fe xxv, a signature of hot gas.

5. The extended soft X-ray emission found in many objects shows a spectrum consistent with a thermal emission spectrum with an abundance pattern with enhanced $\alpha$ elements relative to iron, as expected for an interstellar medium enriched by core collapse $\mathrm{SNe}$ produced in a starburst.

6. A comparison between the soft X-ray spectra of starburst galaxies and galaxies containing Compton thick AGN shows that, on average, the latter show stronger Si XIII emission at $1.85 \mathrm{keV}$ which may be due to a contribution of AGN photoionized gas besides thermal emission from a starburst.

Acknowledgements. This research has made use of software packages CIAO provided by the Chandra X-ray Center (CXC) and FTOOLS provided by NASAS's HEASARC. This research has also made use of the NASA/IPAC Extragalactic Database (NED), which is operated by the Jet Propulsion Laboratory, California Institute of Technology, under contract with the National Aeronautics and Space Administration. The Chandra data archive is maintained by the Chandra X-ray Center at the Smithsonian Astrophysical Observatory. D.B.S. acknowledges support from Chandra grants GO6-7098X and GO78108A. Vivian U also acknowledges support from a NASA Jenkins Predoctoral Fellowship and a SAO Harvard-Smithsonian Predoctoral Fellowship. We thank the referee for helpful comments.

\section{References}

Anders, E., \& Grevesse, N. 1989, Geochim. Cosmochim. Acta, 53, 197 Armus, L., Charmandaris, V., Bernard-Salas, J., et al. 2007, ApJ, 656, 148 Armus, L., Mazzarella, J. M., Evans, A. S., et al. 2009, PASP, 121, 559 Baan, W. A., \& Klöckner, H.-R. 2006, A\&A, 449, 559

Balestra, I., Boller, T., Gallo, L., Lutz, D., \& Hess, S. 2005, A\&A, 442, 469

Ballo, L., Braito, V., Della Ceca, R., et al. 2004, ApJ, 600, 634

Bassani, L., Dadina, M., Maiolino, R., et al. 1999, ApJS, 121, 473

Bianchi, S., Chiaberge, M., Piconcelli, E., Guainazzi, M., \& Matt, G. 2008, MNRAS, 386, 105

Braito, V., Della Ceca, R., Piconcelli, E., et al. 2004, A\&A, 420, 79 Buote, D. A., \& Fabian, A. C. 1998, MNRAS, 296, 977

Burston, A. J., Ward, M. J., \& Davies, R. I. 2001, MNRAS, 326, 403 Bushouse, H. A., Borne, K. D., Colina, L., et al. 2002, ApJS, 138, 1 Chapman, S. C., Blain, A. W., Smail, I., \& Ivison, R. J. 2005, ApJ, 622, 772 Charmandaris, V., Stacey, G. J., \& Gull, G. 2002, ApJ, 571, 282 Chevalier, R. A., \& Clegg, A. W. 1985, Nature, 317, 44

Clements, D. L., McDowell, J. C., Shaked, S., et al. 2002, ApJ, 581, 974

Condon, J. J., Helou, G., Sanders, D. B., \& Soifer, B. T. 1990, ApJS, 73, 359

de Vaucouleurs, G., de Vaucouleurs, A., Corwin, H. G., Jr., et al. 1991, Vol. 1-3, XII, 2069 (Berlin Heidelberg, New York: Springer-Verlag) Della Ceca, R., Ballo, L., Tavecchio, F., et al. 2002, ApJ, 581, L9 Downes, D., \& Eckart, A. 2007, A\&A, 468, L57

Duc, P.-A., Mirabel, I. F., \& Maza, J. 1997, A\&AS, 124, 533 Dudley, C. C. 1999, MNRAS, 307, 553

Dudley, C. C., \& Wynn-Williams, C. G. 1997, ApJ, 488, 720

Dupke, R. A., \& Arnaud, K. A. 2001, ApJ, 548, 141

Elvis, M., Wilkes, B. J., McDowell, J. C., et al. 1994, ApJS, 95, 1

Fabbiano, G., Baldi, A., King, A. R., et al. 2004, ApJ, 605, L21

Farrah, D., Afonso, J., Efstathiou, A., et al. 2003, MNRAS, 343, 585

Farrah, D., Bernard-Salas, J., Spoon, H. W. W., et al. 2007, ApJ, 667, 149

Franceschini, A., Braito, V., Persic, M., et al. 2003, MNRAS, 343, 1181

Gallagher, S. C., Brandt, W. N., Chartas, G., Garmire, G. P., \& Sambruna, R. M. 2002, ApJ, 569, 655

Gallagher, S. C., Schmidt, G. D., Smith, P. S., et al. 2005, ApJ, 633, 71

Gilfanov, M., Grimm, H.-J., \& Sunyaev, R. 2004, MNRAS, 347, L57

Goldader, J. D., Goldader, D. L., Joseph, R. D., Doyon, R., \& Sanders, D. B. 1997a, AJ, 113, 1569 
Goldader, J. D., Joseph, R. D., Doyon, R., \& Sanders, D. B. 1997b, ApJ, 474, 104

Gonçalves, A. C., Véron-Cetty, M.-P., \& Véron, P. 1999, A\&AS, 135, 437 Griffiths, R. E., Ptak, A., Feigelson, E. D., et al. 2000, Science, 290, 1325 Grimes, J. P., Heckman, T., Strickland, D., \& Ptak, A. 2005, ApJ, 628, 187 Grimm, H.-J., Gilfanov, M., \& Sunyaev, R. 2003, MNRAS, 339, 793 Heckman, T. M., Armus, L., \& Miley, G. K. 1990, ApJS, 74, 833 Hinshaw, G., Weiland, J. L., Hill, R. S., et al. 2009, ApJS, 180, 225 Hopkins, P. F., Hernquist, L., Cox, T. J., et al. 2006, ApJS, 163, 1 Howell, J. H., Armus, L., Mazzarella, J. M., et al. 2010, ApJ, 715, 572 Ikebe, Y., Leighly, K., Tanaka, Y., et al. 2000, MNRAS, 316, 433 Imanishi, M., Terashima, Y., Anabuki, N., \& Nakagawa, T. 2003, ApJ, 596, L167 Imanishi, M., Dudley, C. C., \& Maloney, P. R. 2006, ApJ, 637, 114 Inami, H., Armus, L., Surace, J. A., et al. 2010, AJ, 140, 63 Iwasawa, K., \& Comastri, A. 1998, MNRAS, 297, 1219 Iwasawa, K., Sanders, D. B., Evans, A. S., et al. 2005, MNRAS, 357, 565 Iwasawa, K., Sanders, D. B., Evans, A. S., et al. 2009, ApJ, 695, L103 Iwasawa, K., Mazzarella, J. M., Surace, J. A., et al. 2011, A\&AS, in press Kalberla, P. M. W., Burton, W. B., Hartmann, D., et al. 2005, A\&A, 440, 775 Kim, D.-C., Veilleux, S., \& Sanders, D. B. 1998, ApJ, 508, 627 Komossa, S., Burwitz, V., Hasinger, G., et al. 2003, ApJ, 582, L15 Kondratko, P. T., Greenhill, L. J., \& Moran, J. M. 2006, ApJ, 652, 136 Lehmer, B. D., Brandt, W. N., Alexander, D. M., et al. 2008, ApJ, 681, 1163 Lutz, D., Veilleux, S., \& Genzel, R. 1999, ApJ, 517, L13

Marconi, A., Risaliti, G., Gilli, R., et al. 2004, MNRAS, 351, 169

Mazzarella, J. M., et al. 2010, AJ, submitted

Mazzarella, J. M., et al. 2011, AJ, submitted

McDowell, J. C., Clements, D. L., Lamb, S. A., et al. 2003, ApJ, 591, 154

Mirabel, I. F., \& Sanders, D. B. 1987, ApJ, 322, 688

Modica, F., et al. 2010, AJ, submitted

Mould, J. R., Huchra, J. P., Freedman, W. L., et al. 2000, ApJ, 529, 786

Muñoz Marín, V. M., González Delgado, R. M., Schmitt, H. R., et al. 2007, AJ, 134,648

Murphy, T. W., Jr., Armus, L., Matthews, K., et al. 1996, AJ, 111, 1025

Netzer, H., Lemze, D., Kaspi, S., et al. 2005, ApJ, 629, 739

Nomoto, K., Hashimoto, M., Tsujimoto, T., et al. 1997, Nucl. Phys. A, 616, 79

Pérez-Torres, M. A., Mattila, S., Alberdi, A., et al. 2007, ApJ, 671, L21

Persic, M., \& Rephaeli, Y. 2002, A\&A, 382, 843

Petric, A. O., Armus, L., Howell, J., et al. 2011, ApJ, 730, 28

Pietsch, W., Roberts, T. P., Sako, M., et al. 2001, A\&A, 365, L174

Ptak, A., Heckman, T., Levenson, N. A., Weaver, K., \& Strickland, D. 2003, ApJ, 592,782

Ranalli, P., Comastri, A., \& Setti, G. 2003, A\&A, 399, 39

Ranalli, P., Comastri, A., Origlia, L., \& Maiolino, R. 2008, MNRAS, 386, 1464

Risaliti, G., Gilli, R., Maiolino, R., \& Salvati, M. 2000, A\&A, 357, 13

Risaliti, G., Sani, E., Maiolino, R., et al. 2006a, ApJ, 637, L17

Risaliti, G., Maiolino, R., Marconi, A., et al. 2006b, MNRAS, 365, 303

Sanders, D. B., \& Mirabel, I. F. 1996, ARA\&A, 34, 749

Sanders, D. B., Mazzarella, J. M., Kim, D.-C., Surace, J. A., \& Soifer, B. T. 2003, AJ, 126, 1607 (RBGS)

Spoon, H. W. W., Koornneef, J., Moorwood, A. F. M., Lutz, D., \& Tielens, A. G. G. M. 2000, A\&A, 357, 898

Spoon, H. W. W., Marshall, J. A., Houck, J. R., et al. 2007, ApJ, 654, L49

Strickland, D. K., \& Stevens, I. R. 2000, MNRAS, 314, 511

Strickland, D. K., \& Heckman, T. M. 2007, ApJ, 658, 258

Suchkov, A. A., Berman, V. G., Heckman, T. M., \& Balsara, D. S. 1996, ApJ, 463,528

Surace, J. A., Sanders, D. B., \& Mazzarella, J. M. 2004, AJ, 127, 3235

Teng, S. H., Wilson, A. S., Veilleux, S., et al. 2005, ApJ, 633, 664

Teng, S. H., Veilleux, S., Anabuki, N., et al. 2009, ApJ, 691, 261

Tenorio-Tagle, G., \& Munoz-Tunon, C. 1998, MNRAS, 293, 299

Tomisaka, K., \& Ikeuchi, S. 1988, ApJ, 330, 695

Torrejón, J. M., Schulz, N. S., Nowak, M. A., \& Kallman, T. R. 2010, ApJ, 715, 947

Turner, T. J., \& Kraemer, S. B. 2003, ApJ, 598, 916

van der Werf, P. P., Isaak, K. G., Meijerink, R., et al. 2010, A\&A, 518L, 42

Veilleux, S., \& Osterbrock, D.. E. 1987, ApJS, 63, 295 (VO)

Veilleux, S., Kim, D.-C., Sanders, D. B., Mazzarella, J. M., \& Soifer, B. T. 1995, ApJS, 98, 171

Veilleux, S., Kim, D.-C., \& Sanders, D. B. 1999, ApJ, 522, 113

Veilleux, S., Cecil, G., \& Bland-Hawthorn, J. 2005, ARA\&A, 43, 769

Veilleux, S., Rupke, D. S. N., Kim, D.-C., et al. 2009, ApJS, 182, 628

Vignati, P., Molendi, S., Matt, G., et al. 1999, A\&A, 349, L57

Wegner, G., Bernardi, M., Willmer, C. N. A., et al. 2003, AJ, 126, 2268

White, N. E., Swank, J. H., \& Holt, S. S. 1983, ApJ, 270, 711

Xia, X. Y., Xue, S. J., Mao, S., ET AL. 2002, ApJ, 564, 196

Yuan, T.-T., Kewley, L. J., \& Sanders, D. B. 2010, ApJ, 709, 884 (YKS)
Zenner, S., \& Lenzen, R. 1993, A\&AS, 101, 363

Zezas, A., Ward, M. J., \& Murray, S. S. 2003, ApJ, 594, L31

\section{Appendix A: Notes on individual objects}

IRAS F01364-1042: The optical emission-line spectrum is consistent with a shock-heated, LINER spectral type, similar to the extended optical emission in Arp 220 and NGC 6240. The detected X-ray emission is too faint to infer its origin. A point-like hard X-ray source is located at the nucleus of the galaxy. The soft $\mathrm{X}$-ray emission is elongated along the SE-NW direction, possibly due to two blobs straddling the nuclear source.

ESO 203-IG1: This is the only object in our sample from which no X-ray counts are detected. There are two faint X-ray sources to the SSW (15") and NWW (20"), which have optical counter parts in the HST-ACS image that are much fainter than ESO 203IG1 and unrelated to the IRAS galaxy.

VII Zw 31: The soft X-ray image shows a relatively symmetric source with a slight elongation along the NW-SE direction. There are two faint spurs extending to the NW. This is a "typical" soft X-ray source showing an exponential radial brightness profile. A strong $\mathrm{Mg} \mathrm{XI}$ line at $1.3 \mathrm{keV}$ is visible in the spectrum. The hard X-ray emission is resolved (a compact but not point-like source) with a weaker source at $3^{\prime \prime}$ to the West of the compact nucleus. While this object is not met the X-ray AGN selection criteria, the mid-IR [Ne v] line is detected (Petric et al. 2011).

IRAS F05189-2524: There are two Chandra observations of this galaxy (ObsID 2034, 3432) with $20 \mathrm{ks}$ and $15 \mathrm{ks}$ exposures, respectively. The Chandra results have been published in Ptak et al. (2003) and Grimes et al. (2005). Since this is a bright X-ray source, we only use the ObsID 3432 data, which has a similar exposure time to that of our Cycle 8 targets.

The soft X-ray image shows a faint extensions to the NW and SW, but is dominated by a compact source at the nucleus. The hard X-ray source is point-like. The low surface-brightness envelope is consistent with the wing of the PSF, which is broader than that in the soft X-rays.

The spectrum has a hard X-ray bump, typical of the absorbed emission from an AGN. When a photon index of 1.8 is assumed, the absorbing column density is found to be $N_{\mathrm{H}}=9 \pm 1 \times$ $10^{22} \mathrm{~cm}^{-2}$, by fitting the $3-8 \mathrm{keV}$ spectrum. The absorption correction factor for the $2-10 \mathrm{keV}$ luminosity is 1.7 . A $6.4 \mathrm{keV}$ $\mathrm{Fe} \mathrm{K}$ line is detected at the $2 \sigma$ level.

There is a steep rise of the spectrum below $1.5 \mathrm{keV}$. In analogy to Seyfert 2 galaxies with similar absorption, this is likely due to emission line blends of photoionised gas, unresolved at the CCD resolution.

ESO 255-IG7: This is a triple system of linearly aligned galaxies. All three galaxies are detected with MIPS but the northern galaxy is much brighter than the other two (Mazzarella et al. 2011). The X-ray brightness shows a similar pattern. We tentatively assume that the total IR luminosity is distributed among the three galaxies in the ratio of $\mathrm{N}: \mathrm{C}: \mathrm{S}=8: 3: 1$. The northern galaxy is the brightest and has a softer spectrum than the others. The X-ray spectrum is strangely featureless in the soft X-ray band. The central galaxy, in contrast, shows stronger rest energy emission-lines at $0.85 \mathrm{keV}$ and $1.7 \mathrm{keV}$. Resolved hard $\mathrm{X}$-ray emission is detected from all the three galaxies. The X-ray colour varies gradually from the northern to southern galaxies, with $H R(\mathrm{~N}: \mathrm{C}: \mathrm{S})=-0.64,-0.56,-0.42$. 
IRAS 07251-0248: This galaxy is the highest redshift object in our sample. The X-ray source is very faint, detected only in the soft band.

ESO 60-IG16: A compact X-ray source is located at the nucleus of the NE galaxy of this galaxy pair, as is the mid/far-IR emission seen in the MIPS image (Mazzarella et al. 2011). The soft X-ray image shows a faint tail to the SW of length 7", while the hard X-ray emission is point-like. The X-ray spectrum is typical of Seyfert 2 galaxies with an excess in the hard band due to transmitted emission from an AGN absorbed by $N_{\mathrm{H}} \sim 1 \times 10^{23} \mathrm{~cm}^{-2}$. The absorption-corrected 2-10 keV luminosity, $10^{42} \mathrm{erg} \mathrm{s}^{-1}$, is in the range of Seyfert nuclei. The weakness of the soft X-ray emission is partly due to the large Galactic extinction (see Table 2).

IRAS F08572+3915: The northwest nucleus of this double system is suspected to have an heavily obscured AGN, as the Spitzer IRS spectrum shows a very deep silicate absorption and little PAH emission (e.g., Spoon et al. 2007). The northwest nucleus is detected only in the hard band, $(2-4 \mathrm{keV})$, with a small number of counts $(\sim 10)$. There is a faint, soft X-ray source near the southeast nucleus but its physical association is unclear. The hard X-ray colour $(H R=+0.57)$ of the northwest nucleus strongly suggests the presence of an obscured AGN. However, the very small hard X-ray to IR luminosity ratio, $\log (H X / I R)=-4.4$, points to a Compton-thick AGN, although the limited X-ray counts do not allow us to confirm it with the Fe $\mathrm{K}$ line detection. The Chandra result is also reported in Teng et al. (2009).

IRAS 09022-3615: The X-ray spectrum is flat $(H R=-0.17)$ and the $2-10 \mathrm{keV}$ luminosity is $10^{42} \mathrm{erg} \mathrm{s}^{-1}$, which qualifies this galaxy as an AGN. However, the hard X-ray source is marginally resolved. The [Ne v] line is not detected (Farrah et al. 2007).

IRAS F09111-1007: Two galaxies with projected separation of $40^{\prime \prime}$ are both MIPS sources; however, the western galaxy, which is less luminous in the optical, is a stronger $24 \mu \mathrm{m}$ source. HIRES processing of the IRAS image (Surace et al. 2004) suggests that the two galaxies each make comparable contributions to the IRAS flux, contrary to some previous suggestions (Murphy et al. 1996; Duc et al. 1997; Goncalves et al. 1999). In the X-ray, the eastern galaxy is brighter, but the western galaxy has a harder spectrum although the HR does not meet our criterion for AGN selection. The spectrum of the eastern galaxy shows strong Si XIII (see Table 9).

UGC 4881: This system is a pair of galaxies separated by $11^{\prime \prime}$. The eastern component is brighter in the IR and radio than the western component. Howell et al. (2010) estimated the IRAS luminosity ratio of the E-W pair is $\sim 2: 1$, based on the SpitzerMIPS data. SN1999 GW, reported at the position (RA, Dec) $=\left(09^{\mathrm{h}} 15^{\mathrm{m}} 54.7^{\mathrm{s}},+44^{\mathrm{d}} 19^{\mathrm{m}} 55^{\mathrm{s}}\right)_{\mathrm{J} 2000}$, is located near the western nucleus. Three X-ray counts are detected at the position of the supernova in our $15 \mathrm{ks}$ exposure, but the association of the Xrays with the $\mathrm{SN}$ is not clear given the limited counts.

$\mathrm{X}$-ray emission is detected both from the eastern and western nuclei. The eastern componet is brighter and relatively peaky at the nucleus while the X-ray source for the western galaxy is diffuse and has a rather flat brightness distribution. The X-ray spectrum is soft with $H R=-0.73,-0.88$.

UGC 5101: The hard X-ray spectrum $(H R=-0.29 \pm 0.05)$ and the detection of a cold Fe K line with XMM-Newton demostrate that this galaxy contains an obscured AGN (Imanishi et al. 2003). A high-ionization Fe K line might be present, but its detection is below $2 \sigma$ in the Chandra data. Strong Si XIII (Table 9) as well as S XIV needs a large enhancement in abundance of these metals in order to be explained by a thermal emission spectrum alone. A significant contribution of photoionized gas from an AGN would better explain these spectral features. The same Chandra data have been published in Ptak et al. (2003) and Grimes et al. (2005).

ESO 374-IG32: This galaxy pair hosts an OH megamaser (Baan \& Klöckner 2006). The X-ray source is compact but resolved. The soft X-ray emission is elongated in the NE-SW direction while the hard X-ray source has a faint spur to the south.

IRAS F10173+0828: This edge-on galaxy hosts a mega-maser source (Mirabel \& Sanders 1987), and is expected to have an heavily obscured source that powers the large IR luminosity (Goldader et al. 1997a,b). A very faint X-ray source (10 cts) is found at the nucleus only in the soft band.

IRAS F10565+2448: The western galaxy is the IRAS source as it is the only source in the MIPS $24 \mu \mathrm{m}$ image (Mazzarella et al. 2011), and the X-ray emission also comes only from the western galaxy. Much of the X-ray emission arises from the nucleus as indicated by a peaky X-ray distribution. The hard X-ray emission is point-like and the soft X-ray emission has an extension up to $7^{\prime \prime}\left(\sim r_{\max }\right)$. There is a weaker point-like X-ray source $12^{\prime \prime}$ to the south $\left(10^{\mathrm{h}} 59^{\mathrm{m}} 18.48^{\mathrm{s}}, 24^{\mathrm{d}} 32^{\mathrm{m}} 23.3^{\mathrm{s}}, \mathrm{J} 2000\right)$, for which the counterpart is not visible in the HST-ACS I-band image, but it is possibly detected in the Spitzer-IRAC bands. It is probably a background AGN.

NGC 3690 (=Arp 299): This nearby pair of galaxies are often referred to as Arp 299. Although the easten galaxy is referred to as NGC 3690 and the westen galaxy as IC 694 by several authors, here we follow the RBGS and NED conventions and refer to the two galaxies as NGC 3690 East (E) and NGC 3690 West (W). Both galaxies are strong infrared sources. The IRAS flux distribution between the two galaxies estimated by Surace et al. (2004) using the HIRES algorithm is $\mathrm{E}: \mathrm{W}=3: 1$, which is different from that assumed in Iwasawa et al. (2009) based on the $38 \mu \mathrm{m}$ result by Charmandaris et al. (2002). The X-ray to IR luminosity ratios for the eastern and western components are derived using the 3:1 IRAS image ratio.

A detailed study based on a $25 \mathrm{ks}$ Chandra ACIS-I observation (ObSID 1641) has been published by Zezas et al. (2003). In this paper, we used the $10 \mathrm{ks}$ ACIS-S observation. As mentioned in Zezas et al. (2003), there are many discrete sources distributed over the galaxy pair, as well as surrounding diffuse emission. With the overlay on the high-resolution HST-ACS image, association of the discrete X-ray sources with optical counterparts becomes clear in many cases.

Strong Fe K line emission was first detected with BeppoSAX (Della Ceca et al. 2002). A subsequent XMM-Newton observation resolved the two nuclei and found that the spectra of both nuclei show Fe lines but at different energies - at $6.4 \mathrm{keV}$ in NGC $3690 \mathrm{~W}$ and at $6.7 \mathrm{keV}$ in NGC $3690 \mathrm{E}$ (Ballo et al. 2004). The Chandra data alone provide detection of these lines at the $2 \sigma$ level. From the hard band image, the $6.4 \mathrm{keV}$ line should originate from the B1 knot of the eastern galaxy and serves as strong evidence for this region to contain a Compton-thick AGN. We do not consider, contrary to Ballo et al. (2004), that the $6.7 \mathrm{keV}$ immediately implies an AGN (see also Neff et al. 2004 , for radio data), but we do assume that it should have the same origin as that in Arp 220. There are two hard X-ray knots in the eastern galaxy, for which eastern knot has a more absorbed spectrum than the western knot.

The surface brightness profiles are measured separately for the eastern and western galaxies. Because of the proximity of the two galaxies, the measurements are limited within $13^{\prime \prime}$ radius. 
The soft band profile does not reach the $1 \%$ level to define $r_{\max }$, so we do not give the compactness in Table 7.

IRAS F12112+0305: Two X-ray sources aligned in the NE-SW direction with a separation of $\sim 2.5^{\prime \prime}$ are resolved in the Chandra image. They are coincident with the positions of the two optical nuclei of this double system. Both nuclei are hard X-ray sources, with the SW nucleus being slightly brighter and harder in X-ray colour. The quality of the spectrum from this short exposure observation is poor. A faint soft X-ray arm, similar to that seen in Mrk 266 (Mazzarella et al. 2010, and references therein), emanates from the NE nucleus. The same Chandra data are reported in Teng et al. (2005) and the XMM-Newton results are reported in Franceschini et al. (2003) where this object is considered to be starburst dominated.

UGC 8058 (=Mrk 231): Mrk 231 is the only optically classified Seyfert 1 galaxy in our C-GOALS sample and it is also a low-BAL object. The infrared SED is considered to be powered primarily by the AGN (e.g. van der Werf et al. 2010), but note that mid-IR [Ne v] is not detected (Farrah et al. 2007). There are four separate Chandra-ACIS exposures giving a total exposure time of $160 \mathrm{ks}$, making this the longest exposure time by far among the observations of our sample. Results on part or all of these data have been reported in Ptak et al. (2003), Grimes et al. 2004), and Gallagher et al. (2002, 2005).

Almost all of the emission above $2 \mathrm{keV}$ comes from the unresolved nucleus. Despite the Seyfert 1 optical classification, the $\mathrm{X}$-ray spectrum does not at all resemble that which is typically observed in Seyfert 1 galaxies, i.e., a steep $(\Gamma \sim 2)$ power-law with or without weak absorption by partially ionized gas (the "warm" absorber). The Mrk 231 spectrum above $3 \mathrm{keV}$ is flat $(\Gamma \simeq 0.7)$. A Fe $\mathrm{K}$ feature is visible at $6.4 \mathrm{keV}(E W \sim 100 \mathrm{eV})$, in the $160 \mathrm{ks}$ exposure spectrum, similar to that previously seen in the XMM-Newton data (Franceschini et al. 2003; Turner \& Kraemer 2003; Braito et al. 2004). Given the detection of an hard X-ray excess with the BeppoSAX PDS (Braito et al. 2004), the nucleus is considered to host a heavily obscured AGN with an absorbing column of $N_{\mathrm{H}} \sim 2 \times 10^{24} \mathrm{~cm}^{-2}$. The flat 3-7 keV continuum is attributed to the reflected AGN continuum. The constant flux measured in the four separate observations (Gallagher et al. 2005) is compatible with this interpretation. However, the observed $\mathrm{EW}$ of the Fe $\mathrm{K}$ line is much smaller than the expected nominal value.

The soft X-ray emission also peaks strongly at the nucleus. This causes $r_{\max }$ to be only $5^{\prime \prime}$, because of how this parameter is defined (the radius where the brightness falls to $1 \%$ of the peak brightness).

A large, low surface brightness extension of more than $30^{\prime \prime}$ (=25 kpc) down to the $2 \times 10^{-17} \mathrm{erg} \mathrm{s}^{-1} \mathrm{~cm}^{-2} \operatorname{arcsec}^{-2}$ level is detected in the deep X-ray image. Much of the extended emission beyond $5^{\prime \prime}$ from the nucleus is emitted below $1 \mathrm{keV}$. Between $1 \mathrm{keV}$ and $2 \mathrm{keV}$, the emission is due predominantly to emission lines of Mg XI and Si XIII (see Fig. 13 in Sect. 5.1.4).

The deep X-ray exposure reveals a horse-shoe structure $15^{\prime \prime}$ south of the nucleus (indicated by an arrow in Fig. 2), where a faint optical counterpart is visible in the HST image. Note this is not the UV horse-shoe structure with star forming knots, located closer $\left(\sim 4^{\prime \prime}\right)$ to the nuclues, imaged in the optical/UV (MuñozMarin et al. 2007).

With the long exposure time, a reasonably good quality spectrum of the extended, low surface-brightness emission was obtained. The temperature implied from the fit to this very extended low surface brightness emission $(k T \simeq 0.5 \mathrm{keV})$ is lower than the spatially averaged values for other galaxies and little emission is

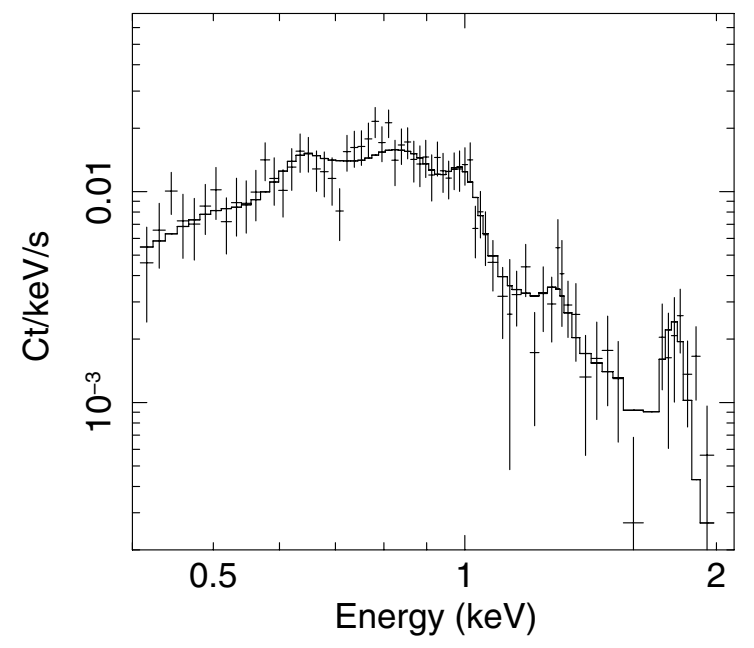

Fig. A.1. The Chandra ACIS spectrum of the extended, low surface brightness emission around Mrk 231, fitted with a thermal emission model.

detected above $2 \mathrm{keV}$. The spectrum fitted with the model above is also shown for comparison (Fig. A.1). The diameter of the nebula is $\sim 50 \mathrm{kpc}$.

IRAS 13120-5453: The X-ray source is compact both in the soft and hard bands. The radial brightness profile is slightly broader than the PSF but the extension is limited below $1^{\prime \prime}$. The hard $\mathrm{X}$-ray colour $(H R=-0.13)$ classifies this object as an AGN. The large Galactic extinction (Table 2) is part of the reason for the hard X-ray colour. The small hard X-ray to IR ratio, $\log (\mathrm{HX} / \mathrm{IR})<-4$, places this object as a Compton thick AGN candidate (Table 6).

VV 250: This is a widely separated galaxy pair with a clear tidal structure conneting the two galaxies. The eastern galaxy is brighter in X-rays than the western galaxy, with a count ratio of $\sim 10: 1$. The IRAS HIRES estimate gives a flux ratio of $\mathrm{E}: \mathrm{W}=0.85: 0.15$ (Surace et al. 2004). The hard X-ray core is marginally resolved. A broad Fe L bump is seen below $1 \mathrm{keV}$ in the spectrum, but the other emission lines, e.g., Si XIII, do not stand out. The X-ray emission associated with the Western galaxy is patchy and distributed roughly towards the direction of the Eastern galaxy.

UGC 8387 (=IC 883): The soft X-ray emission is elongated along the minor axis of the galaxy's main body, with faint filaments forming a loop on the NW side. There is a relatively sharp boundary in the soft X-ray emission on the SE side. A compact, but resolved hard X-ray source is located at the nuclear position, which produces a faint tail in the energy spectrum above $4 \mathrm{keV}$. There is a compact soft X-ray source about $9^{\prime \prime}$ to the SE of the galaxy nucleus, which has an optical counterpart in the HST-ACS image, most likely a star cluster. The total counts detected from this object is $15.8 \pm 4.0 \mathrm{cts}$ in the $0.4-7 \mathrm{keV}$ band. The X-ray color, $H R=-0.64 \pm 0.30$, suggests that the X-ray spectrum is softer than the source associated with the galaxy main body. The details of this galaxy together with the other GOALS datasets are discussed in a separate paper (Modica et al. 2010). Very faint [Ne v] is detected in the Spitzer IRS high resolution spectrum, which indicates that a weak AGN might be present in this galaxy. The X-ray spectrum of this object is one of those exhibiting enhanced Si XIII emission (Table 9).

UGC 8696 (=Mrk 273): A detailed analysis of the Chandra data for this ULIRG, which has an absorbed AGN, has been published in Xia et al. (2002). Analyses of the same data set 
are also given in Ptak et al. (2003), Satyapal et al. (2004), Grimes et al. (2005), and González-Martín et al. (2006). The XMM-Newton results have been reported by Balestra et al. (2005). The absorbing column density towards the X-ray source is $N_{\mathrm{H}} \sim 4 \times 10^{23} \mathrm{~cm}^{-2}$, and the absorption corrected $2-10 \mathrm{keV}$ luminosity is $0.9 \times 10^{43} \mathrm{erg} \mathrm{s}^{-1}$. A cold Fe $\mathrm{K}$ line at $6.4 \mathrm{keV}$ is detected with $E W \sim 0.24 \mathrm{keV}$. Our analysis of the hard X-ray emission seems to indicate that the absorbed AGN may be located at the southern nucleus. The soft X-ray tail, which emits little above $2 \mathrm{keV}$ and has luminosity of $5.5 \times 10^{40} \mathrm{erg} \mathrm{s}^{-1}$, extends to the south. This X-ray emission is, however, not associated with the tidal tail imaged in the optical, but rather is located behind it. The details concerning the nuclear position, the nature of the double nuclei, and the soft X-ray tail can be found in Iwasawa et al. (2011).

IRAS F14348-1447: This is a double system with two nuclei separated by $\sim 4^{\prime \prime}$. In the IRAC bands, the southern nucleus is brighter (Mazarella et al. 2010b), while the MIPS image is unresolved. We assumed the northern and southern nuclei to have IR flux ratio of $\mathrm{N}: \mathrm{S}=1: 3$. The southern nucleus is also a brighter $\mathrm{X}$-ray source and shows point-like hard X-ray emission. While the HR value for the total emission is -0.48 , when selecting only the southern nuclear region, $H R=-0.18 \pm 0.19$, indicating the possible presence of an AGN, which is possibly Compton thick, based on the small HX/IR ratio (Table 6). Imanishi et al. (2007) present Spitzer IRS spectra and also suggest the presence of an AGN based on the $L$-band spectrum. The northern nucleus is enveloped by soft X-ray emission. Based on the XMM-Newton data, Franceschini et al. (2003) classified this galaxy as a starburst dominated source.

IRAS F14378-3651: A faint, point-like source is detected at the nucleus of this galaxy in the hard band while there is an additional fainter blob (or an extension) at $3^{\prime \prime}$ to the west, which is also detected in the soft X-ray band. The hard X-ray colour $(H R=-0.18)$ classifies this object as an AGN with a relatively low 2-10 keV luminosity of $\sim 3 \times 10^{41} \mathrm{erg} \mathrm{s}^{-1}$, which agrees with the Seyfert 2 classification derived from the optical spectroscopy (Kim et al. 1998). The small HX/IR ratio means this object is a Compton thick AGN candidate (Table 6). There is a faint structure in the HST-ACS image, which might be a star cluster within the NW extended structure noted by Bushouse et al. (2002).

VV 340: This galaxy pair consists of an edge-on galaxy to the north and a face-on galaxy to the south. The IRAS HIRES estimate yields a flux ratio N:S $=0.85: 0.15$ (Surace et al. 2004). The northern galaxy is brighter in the $24 \mu \mathrm{m}$ image and the detection of [Ne v] in the IRS spectrum plus the Chandra spectrum with a possible $6.4 \mathrm{keV}$ line (see below) suggest the presence of a heavily obscured AGN (see Armus et al. 2009, for the GOALS multi-wavelength datasets on this object). The northern galaxy is brighter in X-rays than the southern galaxy. The soft X-ray emission of the northern galaxy shows a complex morphology and has a flat brightness profile. It extends along the minor axis of the edge-on galaxy, suggesting a relation with a nuclear outflow. The soft X-ray peak is displaced by $2^{\prime \prime}$ to NE from the nuclear position, where the hard X-ray emission peaks. This is reflected in the surface brightness profile, with the innermost bin being depressed. Absorption at the nucleus is a probable cause.

While the hardness ratio, $H R=-0.74 \pm 0.08$, indicates a soft spectrum, there is a small bump above $4 \mathrm{keV}$ which peaks at the rest-energy of $6.4 \mathrm{keV}$. The quality of the data is insufficient to warrant a significant detection of the Fe K line at $6.4 \mathrm{keV}(\sim 2 \sigma)$, but the spectral shape is strongly suggetive of a heavily obscured, possibly Compton-thick AGN. The absorbing column density has to be close to $10^{24} \mathrm{~cm}^{-2}$. The hard X-ray source is resolved into a nuclear source and small blobs located both North and South up to 7 " away, which are in alignment with the radio morphology (Condon et al. 1990). A strong Si XIII ( $E W \sim 0.3 \mathrm{keV})$ at $1.87 \mathrm{keV}$ is noted in the soft band spectrum, which could be partly due to extended gas photoionied by the AGN in addition to thermal emission from a starburst. The Chandra data has been published as a part of the water maser sample of Kondratko et al. (2006).

The X-ray emission associated with the southern galaxy has no well-defined peak and shows a patchy brightness distribution. An inspection of the X-ray and optical images suggests that the X-ray emission in this galaxy may be composed of multiple $\mathrm{X}$-ray sources associated with individual star clusters or giant $\mathrm{H}$ II regions within this galaxy.

VV 705 (=I Zw 107): This system contains a close pair of galaxies with clear tidal tails. The northern galaxy dominates the IRAS flux. While X-ray emission is detected from both galaxies, the northern source is also much brighter in X-rays $(\mathrm{N}: \mathrm{S}=$ $8: 1)$. A bright core is found at the northern nucleus with a faint arc to the NE that has a flat brightness distribution. There are two faint sources to the NW at $(\mathrm{RA}, \mathrm{Dec})=\left(15^{\mathrm{h}} 18^{\mathrm{m}} 05.22^{\mathrm{s}}\right.$, $\left.+42^{\mathrm{d}} 44^{\mathrm{m}} 58.23^{\mathrm{s}}\right)_{\mathrm{J} 2000}$ and to the W $($ RA, Dec $)=\left(15^{\mathrm{h}} 18^{\mathrm{m}} 05.05^{\mathrm{s}}\right.$, $\left.42^{\mathrm{d}} 44^{\mathrm{m}} 47.53^{\mathrm{s}}\right)_{\mathrm{J} 2000}$, which are probably background sources.

IRAS F15250+3608: With only a $10 \mathrm{ks}$ exposure, a weak soft $\mathrm{X}$-ray source is detected. No emission is detected above $2 \mathrm{keV}$, which implies an extremely hard X-ray limit compared to the IR luminosity, $\log \left(L_{\mathrm{HX}} / L_{\mathrm{IR}}\right)<-5$. The Chandra result has been reported in Teng et al. (2005) while the XMM-Newton results are published in Franceschini et al. (2003).

UGC 9913 (=Arp 220): This object, the nearest ULIRG, has been studied extensively at all wavelengths. The hard X-ray emission is elongated across the two nuclei with a separation of 1 ", and is peaked at the western nucleus (Clements et al. 2002; Iwasawa et al. 2005). The strong, Fe xxv emission, detected in XMM-Newton (Iwasawa et al. 2005) and Suzaku (Teng et al. 2009) spectra, should come from this region. The line detection in the Chandra data is barely $2 \sigma$ (Clements et al. 2002, and Table 10). The region around the western nucleus within 2.7" shows a hard X-ray colour, $H R=-0.16$, which is in the range observed for AGN. However, absorption, rather than an intrinsicaly hard spectrum of an AGN, plays an important role in making the spectrum appear hard.

The soft X-ray peak is displaced to the NW from the western nucleus where the hard X-ray emission is peaked. This is primarily due to absorption in the nuclear region, which leads to the dip at the innermost bin of the soft X-ray radial profile. The abrupt drop of the surface brightness at 3-4" NW from the nucleus is also due to absorption which is caused by the dust lane running across the nucleus. Towards the SE, a relatively flat soft X-ray tail is seen up to $10^{\prime \prime}$, where the brightness drops sharply.

The low surface brightness emission extending beyond the inner bright part emits little emission above $2 \mathrm{keV}$. The soft Xray nebula's extension is up to $\sim 45^{\prime \prime}$ arcsec in the E-W direction. Both ends seem to show a looped morphology with a welldefined edge. The same data have appeared in Ptak et al. (2003), McDowell et al. (2003) and Grimes et al. (2005).

ESO 69-IG6: This system is a widely separated $\left(\sim 60^{\prime \prime}\right)$ pair, and the northern galaxy likely accounts for almost all the IRAS flux, as it is the only source in the MIPS image (Mazzarella et al. 2011). The X-ray source properties described in this paper are only for this northern galaxy. The northern galaxy shows an intriguing X-ray morphology. The soft X-ray image shows a 
double morphology with diffuse emission, while the hard band shows three compact sources aligned linearly. One of the hard $\mathrm{X}$-ray sources is located inbetween the soft X-ray double peaks. This suggests that the soft X-ray depression between the brightness peaks may be due to absorption. This complex X-ray morphology has no correspondence in the images in the other bands (UV/optical/near-IR). The optical nucleus imaged by the HSTACS is located at the northern blob seen in the soft X-ray band, and this is the apex of the X-ray radial profiles. The X-ray spectrum is soft $(H R=-0.67)$ and shows strong Si XIII (Table 9).

The southern galaxy hosts a point-like, strongly absorbed Xray source with $N_{\mathrm{H}} \sim 4 \times 10^{22} \mathrm{~cm}^{-2}$. The absorption-corrected $2-10 \mathrm{keV}$ luminosity is estimated to be $1.5 \times 10^{42} \mathrm{erg} \mathrm{s}^{-1}$. It is likely a moderately luminous Seyfert 2 nucleus.

NGC 6240: This nearby LIRG has often been considered to be starburst dominated. The presence of an AGN was suggested by the detection of a strong Fe K complex in the ASCA spectrum (Iwasawa \& Comastri 1998), followed by the detection of an hard X-ray excess with BeppoSAX (Vignati et al. 1999) and RXTE (Ikebe et al. 2000), indicating a strongly absorbed $\left(N_{\mathrm{H}} \sim\right.$ $2 \times 10^{24} \mathrm{~cm}^{-2}$ ) AGN. Additional evidence for an AGN was also obtained from infrared spectroscopy (Armus et al. 2006; Risaliti et al. 2006a). Chandra observations resolved the X-ray source into two nuclei and revealed that both nuclei host Comptonthick AGN through the detection of reflection-dominated spectra (Komossa et al. 2003). The same Chandra data are included in Ptak et al. (2003) and Grimes et al. (2005). A detailed analysis of the soft X-ray emission spectrum, using the X-ray grating spectrometer data, is presented by Netzer et al. (2005).

The low surface brightness emission in soft X-rays extends to large radii corresponding to a projected diameter of $\sim 70$ $80 \mathrm{kpc}$. This soft X-ray nebula has a similar morphology to that of other galactic wind signatures like $\mathrm{H} \alpha$ (Heckman et al. 1996; Veilleux et al. 2003). The hard X-ray emission is concentrated at the two nuclei with a projected separation of $1.6^{\prime \prime}$, which are barely separated at the Chandra resolution. The southern nucleus is the brighter hard X-ray source. The X-ray spectrum presented in this paper is taken from the brighter, butterfly-shaped, inner part.

IRAS F17132+5313: Two merging galaxies aligned in the E$\mathrm{W}$ direction make up this system, and both contribute to the IRAS flux. In X-rays, the eastern galaxy is the main X-ray source and a faint extension is seen towards the western galaxy both in the soft and hard X-ray bands. The Chandra spectrum shows strong emission lines of $\mathrm{Ne} I \mathrm{X}(0.9 \mathrm{keV})$ and $\mathrm{Mg}$ XI $(1.3 \mathrm{keV})$. The Si XIII spectral region is noisy. No AGN signatures are detected in optical (Veilleux et al 1995; Yun et al. 2010) or in X-ray, while the mid-IR [Nev] is detected in the Spitzer IRS spectrum (Petric et al. 2011).

IRAS F17207-0014: This galaxy has been observed with Chandra twice, one snapshot with 9 ks (ObsID 4114) and another longer (49 ks) exposure (ObsID 2035). Here, we used the long exposure data. The same data have been published in Ptak et al. (2003), Grimes et al. (2005) and Teng et al. (2005).

The X-ray source has two peaks separated by $\sim 2^{\prime \prime}$. The southern peak is due to hard X-ray emission and coincides with the NED nuclear position. The northern peak is mainly due to soft X-ray emission. The weak soft X-ray emission at the nuclear position means that an excess of absorption is responsible for the suppression. The dip of the soft X-ray surface brightness profile at the innermost $1^{\prime \prime}$, similar to Arp 220, is likely an effect of absorption.
The optical and infrared diagnostics (Veilleux et al. 1999; Lutz et al. 1999; Risaliti et al. 2006b), the XMM-Newton observation (Franceschini et al. 2003) and previous Chandra publications all point to a starburst classification. Our analysis shows the possible presence of strong, high-ionization Fe K (Table 10) on a hard continuum (Table 8), which is, again, similar to Arp 220. IRAS F18293-3413: X-ray emission is detected only at the brighter face-on galaxy in this pair system. The observed X-ray flux is consistent with the BeppoSAX value reported in Risaliti et al. (2000). There is a possible $2-7 \mathrm{keV}$ excess at the position of SN 2004ip (Perez-Torres et al. 2007, RA $=18^{\mathrm{h}} 32^{\mathrm{m}} 41.2^{\mathrm{s}}$, Dec $\left.-34^{\mathrm{d}} 11^{\mathrm{m}} 26.8^{\mathrm{s}}\right)$. However, the position is too close to the nuclear source $\left(\sim 1^{\prime \prime}\right.$ away) to be resolved, given the small detected counts $(6 \mathrm{cts})$. The X-ray source in this galaxy is one of the brightest sources among our $26 \mathrm{Cycle}-8$ targets. The X-ray emission is resolved both in the soft and hard bands (up to $12^{\prime \prime}$ and 7 ", respectively). The soft X-ray emission extends towards the south beyond the main body of the galaxy, suggesting a possible outflow in this direction. The X-ray spectrum shows a strong Si XIII line as well as Mg XI and Fe L blends around $0.9 \mathrm{keV}$.

ESO 593-IG8: A cross-shaped double galaxy system aligned perpendicularly. Dudley (1999) has classified this source as a "PAH galaxy" based on near-IR spectroscopy. The mid-IR spectroscopy detected [Ne v] (Petric et al. 2011). The X-ray emission peaks near the intersection of the two galaxies and shows a curious morphology. The bright core is elongated in the NE-SW direction and soft X-ray emission extends primarily towards the south. The hard X-ray source has a narrow $\sim 10^{\prime \prime}$ tail extending to the south. There is a sharp peak, $r<1^{\prime \prime}$, in the soft X-ray band. The spectrum shows distinct emission-line peaks at 0.95 , 1.8 , and possibly at $0.6 \mathrm{keV}$. Possible identifications for these lines are $\mathrm{Ne}$ IX, $\mathrm{Si}$ XIII and $\mathrm{O}$ VIII, respectively. If the presence of $\mathrm{O}$ VIII is real, this soft X-ray spectrum would be difficult to account for by a keV-temperature thermal spectrum. The Si XIII is strong (Table 9).

IRAS F19297-0406: This is the third most luminous object in the C-GOALS sample but the observed X-ray emission is faint. While the soft X-ray emission shows a slight extension, the hard $\mathrm{X}$-ray emission is point-like.

IRAS 19542+1110: This face-on galaxy has a compact X-ray source with a very hard spectrum. The hard X-ray emission is point-like. The hard X-ray spectrum is consistent with a transmitted AGN continuum absorbed by $N_{\mathrm{H}} \sim 5 \times 10^{22} \mathrm{~cm}^{-2}$. The absorption-corrected $2-10 \mathrm{keV}$ luminosity is estimated to be $6 \times 10^{42} \mathrm{erg} \mathrm{s}^{-1}$. No information on the optical classification is available, most likely due to the low Galactic lattitude $b=-8.9^{\circ}$.

CGCG 448-020 (=II Zw 96): This V-shaped interacting pair has been suggested to host obscured star forming sites. Four principal near-IR peaks are identified by Zenner \& Lenzen (1993) and denoted as A, B, C, and D (also see Fig. 2 in Goldader et al. 1997b). The Spitzer-MIPS image has revealed that the more than 70 per cent of the total $24 \mu \mathrm{m}$ emission comes from D (Inami et al. 2010), which is almost invisible in the B-band image. Source D is the closest to the IRAS peak and is likely to dominate the IRAS flux. There are two compact hard X-ray sources, one of which is slightly elongated encompassing Sources $C+D$ and the other is coincident with Source A. Source C+D has a $\mathrm{X}$-ray colour, $H R=+0.05 \pm 0.14$, which is in the range of AGN, but like the nuclear region of Arp 220, absorption within these red knots is likely the major reason for the hard X-ray colour. There is a spur extending from Source A to the north, which is identified as the base of a soft X-ray filament bending over to the NW. There is another soft X-ray filament extending towards the 
NW. Both filaments appear to connect to a northern soft X-ray blob, which altogether form an elongated loop. The location of the NW soft X-ray blob is displaced from the NW galaxy, and the relationship between the X-ray source and optical galaxy is not clear.

There are three faint sources to the east, aligned linearly and only seen in soft X-rays (Sect. 5.2). Two of these sources have clear optical counterparts in the HST-ACS image. They are luminous star clusters or dwarf galaxies residing within the tidal tail of the merger.

The radial surface brightness profiles were constructed with Source A as the apex. The soft X-ray profile has a power-law form with a slope of $-1.5 \pm 0.1$ out to $17^{\prime \prime}$. The hard X-ray profile has a bump at $4-10^{\prime \prime}$ due to Source $\mathrm{C}+\mathrm{D}$. The X-ray colour map shows that Source D is the hardest X-ray source, which is consistent with an absorbed X-ray source associated with an obscured far-IR source. No clear evidence for an obscured AGN can be found in the X-ray spectrum. A clear line feature at $1.6 \mathrm{keV}$, which is, however not at the energy of Si XIII, remains unidentified. There is a marginally significant line feature at $4.1 \mathrm{keV}$, which also has no obvious identification.

In terms of the $L_{\mathrm{x}}-I R$ correlation (Ranalli et al. 2003), a comparison between Source A and Source $C+D$ may be illuminating. While the hard X-ray luminosities of Source $\mathrm{C}+\mathrm{D}$ and Source A are comparable, the IR luminosity of Source C+D is larger than Source A by factor of $\sim 2$ at $24 \mu \mathrm{m}$ and factor of $\sim 7$ at $70 \mu \mathrm{m}$ (Inami et al. 2010), i.e., within this single object, it is obvious that the $L_{\mathrm{x}}-I R$ correlation does not hold for individual "knots". The powerful, obscured IR Source C+D is X-ray quiet (at a given IR luminosity), compared with the less powerful, unobscured Source A. This is reminiscent of the hard X-ray queiet nature of the HXQ galaxies relative to the nearby star-forming galaxies (Iwasawa et al. 2009).

ESO 286-IG19: A bright elliptical soft X-ray core, elongated along the NE-SW direction, is seen around the nucleus of the galaxy. A faint spur towards the NW is extended up to $10^{\prime \prime}$. The hard X-ray image shows a point like source with a resolved faint extension to the NE up to $3^{\prime \prime}$. The Chandra data have been published in Ptak et al. (2003) and Grimes et al. (2005). Franceschini et al. (2003) classified this object as an AGN based on the XMMNewton data. No AGN signatures were detected in the optical and mid-IR spectra (Table 6).

There is a faint point-like source, visible both in the soft and hard bands, at 7" to the SE from the nucleus. A brighter compact source with a rather hard spectrum, located at $8.5^{\prime \prime}$ to the SSW from the nucleus, is also found. An optical counterpart of this $\mathrm{X}$-ray source is found in the HST-ACS I-band image (see the Chandra overlay in Fig. 2), and is probaly a background AGN.

The X-ray spectrum shows a clear hard X-ray tail with $\Gamma$ 0.7 . While the $\mathrm{X}$-ray colour of the total emission $(H R=-0.77)$ does not qualify as AGN, this hard X-ray emission is the one identifed as an absorbed AGN component in the XMM-Newton spectrum (Franceschini et al. 2003). An iron K line is not detected either in the Chandra or XMM-Newton spectra. As shown in Fig. 12, the relatively good quality soft X-ray spectrum suggests that the $\mathrm{X}$-ray emitting gas is $\alpha$ element rich relative to iron and the metallicity pattern deviates from solar. Strong Si XIII is detected, suggesting a further enhancement of Si metallicity (Table 11), similar to the same line detected in Compton thick AGN (Sect. 5.1.4).

IRAS 21101+5810: A faint, compact X-ray source is detected at the nuclear position. A western extension is seen only in the soft band where no obvious optical counterpart is visible in the ACS image.
ESO 239-IG2: A point-like source is detected at the nucleus in the hard band while the soft band shows a sharp peak at the nucleus with low-brightness emission extending to $10^{\prime \prime}$. The soft $\mathrm{X}$-ray extension is primarily in the $\mathrm{N}-\mathrm{S}$ direction. The spectrum is softer than the sample average $(H R=-0.75)$ with the possible presence of a weak hard X-ray tail.

IRAS F22491-1808: This is a well-known merger system with spectacular tidal tails. Farrah et al. (2003) conclude that more than half of the bolometric luminosity originates from an AGN, based on an SED fit while optical data favour a starburst. The observed X-ray source is faint and most of the counts are detected in the soft band, thus it has a very soft spectrum. No X-ray signature of an AGN can be found in the data. The XMM-Newton data have been presented in Franceschini et al. (2003).

ESO 148-IG2: Two galaxy nuclei are aligned in the N-S direction with a $5^{\prime \prime}$ separation, and the optical image shows a classic double tidal tail. The XMM-Newton data show that this object contains an absorbed AGN based on the X-ray spectrum (Franceschini et al. 2003), with an estimated absorbing column density, $N_{\mathrm{H}} \sim 7 \times 10^{22} \mathrm{~cm}^{-2}$, although the broad PSF of XMMNewton did not identify the location of the AGN. Many pieces of evidence show that the southern nucleus, which is a much brighter source in the Spitzer-MIPS $24 \mu \mathrm{m}$ image (we assumed IR flux ratio of 1:3 for N:S, based on the mid-IR flux ratio estimated by Charmandaris et al. 2002), contains the AGN. The HST-ACS image shows a point-like optical source at the southern nucleus. Risaliti et al. (2006a,b) classified this nucleus as an AGN based on their analysis of the $L$-band spectrum.

Chandra results have been reported previously in Ptak et al. (2003) and Grimes et al. (2005). The Chandra observations clearly demonstrate that the southern nucleus is the hard X-ray source, and the location of the absorbed AGN. When the intrinsic spectral slope is assumed to have $\Gamma=1.8$, the absorbing column density is estimated to be $N_{\mathrm{H}}=(5 \pm 2) \times 10^{22} \mathrm{~cm}^{-2}$. The absorption corrected 2-10 keV luminosity is then $0.8 \times 10^{42} \mathrm{erg} \mathrm{s}^{-1}$.

ESO 77-IG14: This galaxy pair has two similar disk galaxies separated by $\sim 17^{\prime \prime}$, and two Spitzer-MIPS sources with comparable fluxes are detected at the respective nuclei; thus both members are likely to contribute to the IRAS flux. The optical spectral type listed in NED is H II. Two X-ray sources, also with comparable fluxes (1:0.6 in the $0.4-7 \mathrm{keV}$ count ratio for the $\mathrm{NE}$ and SW galaxies). The NE source is harder in X-ray colour. Both X-ray sources are faint but they are not point-like. There is another faint X-ray source $(7.8 \pm 2.8$ cts in the $0.4-7 \mathrm{keV}$ band) located further south, which has a very faint optical counterpart in the ACS image.

IRAS F23365+3604: This galaxy is generally considered to be a heavily obscured object (Burston et al. 2001) and the optical class is LINER (Veilleux et al. 1995). A faint X-ray source is detected at the nucleus. The quality of the spectrum of the short exposure $(10 \mathrm{ks})$ observation is poor, but the hard X-ray colour, $H R=-0.22$, classifies this object as an AGN, which could be Compton thick, based on the small HX/IR ratio (Table 6). The Chandra results are also reported in Teng et al. (2005).

\section{Appendix B: Image extension of the very soft X-ray band}

The 0.4-0.7 keV images of Arp 220 (UGC 9913), ESO 286IG19, NGC 6240 and Mrk 231 (UGC 8058) are shown as a supporting material for footnote 4 , which argues against a spectral 


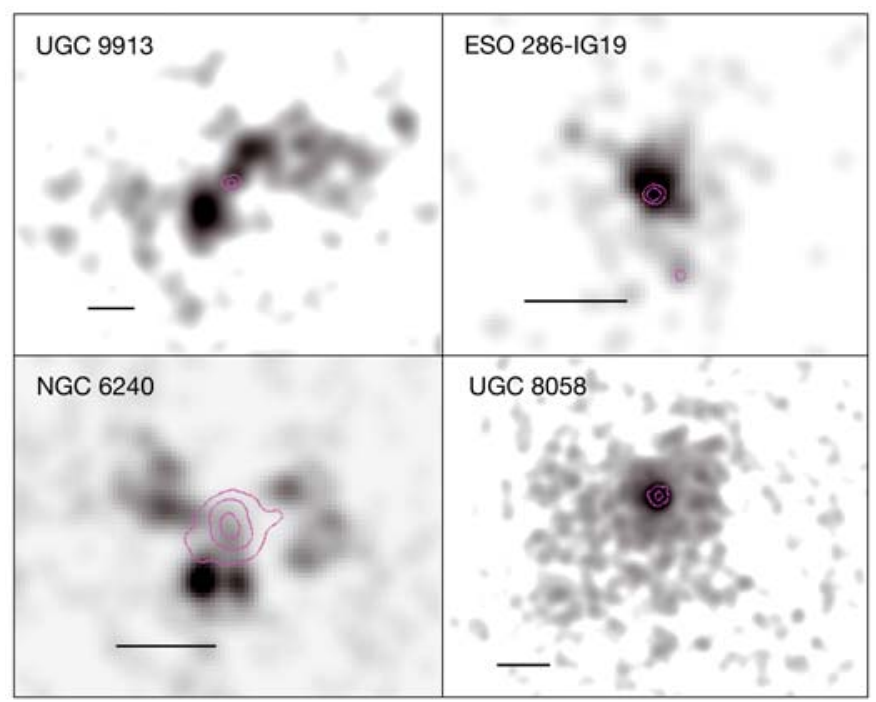

Fig. B.1. The soft band (0.4-0.7 keV), grey scale images of Arp 220, ESO 286-IG19, NGC 6240 and Mrk 231. The hard band (3-7 keV) source in each object is indicated by the purple contours. The scale bar in each panel indicates 5 arcsec. model in which a power-law component accounts for the very soft band (e.g., 0.4-0.7 keV) spectrum as well as the hard band (3-7 keV) in a starburst galaxy. The four objects were selected for their brightness in the $0.4-0.7 \mathrm{keV}$ band. The upper panels for Arp 220 and ESO 286-IG19 are objects in which no clear AGN signature is found in the hard band, and thus relevant to the above argument. The bottom two panels are for NGC 6240 and Mrk 231 in which an AGN component is found in their hard band spectra. In all cases, the soft band $(0.4-0.7 \mathrm{keV})$ emission is significantly extended, while the hard band (3-7 keV) emission is much more compact, as shown in contours. Furthermore, the soft band emission is displaced from the $3-7 \mathrm{keV}$ hard band emission apart from Mrk 231. It should be noted that the unresolved nuclear component of Mrk 231 is always dominant over the whole energy band. In Arp 220 and NGC 6240, it is evident that the soft $\mathrm{X}$-ray emission is suppressed in their nuclear regions where obscuration is substantial and only the hard band emission comes through. As shown in the above examples, the distinct morphology between the $0.4-0.7 \mathrm{keV}$ and 3-7 keV bands means that the origins of the emission in the two bands are different and cannot be represented by a single component in spectral modelling, even if it can provide a good fit. 
K. Iwasawa et al.: C-GOALS survey

\section{Appendix C: X-ray images of the C-GOALS sample}

\section{F01364-1042}
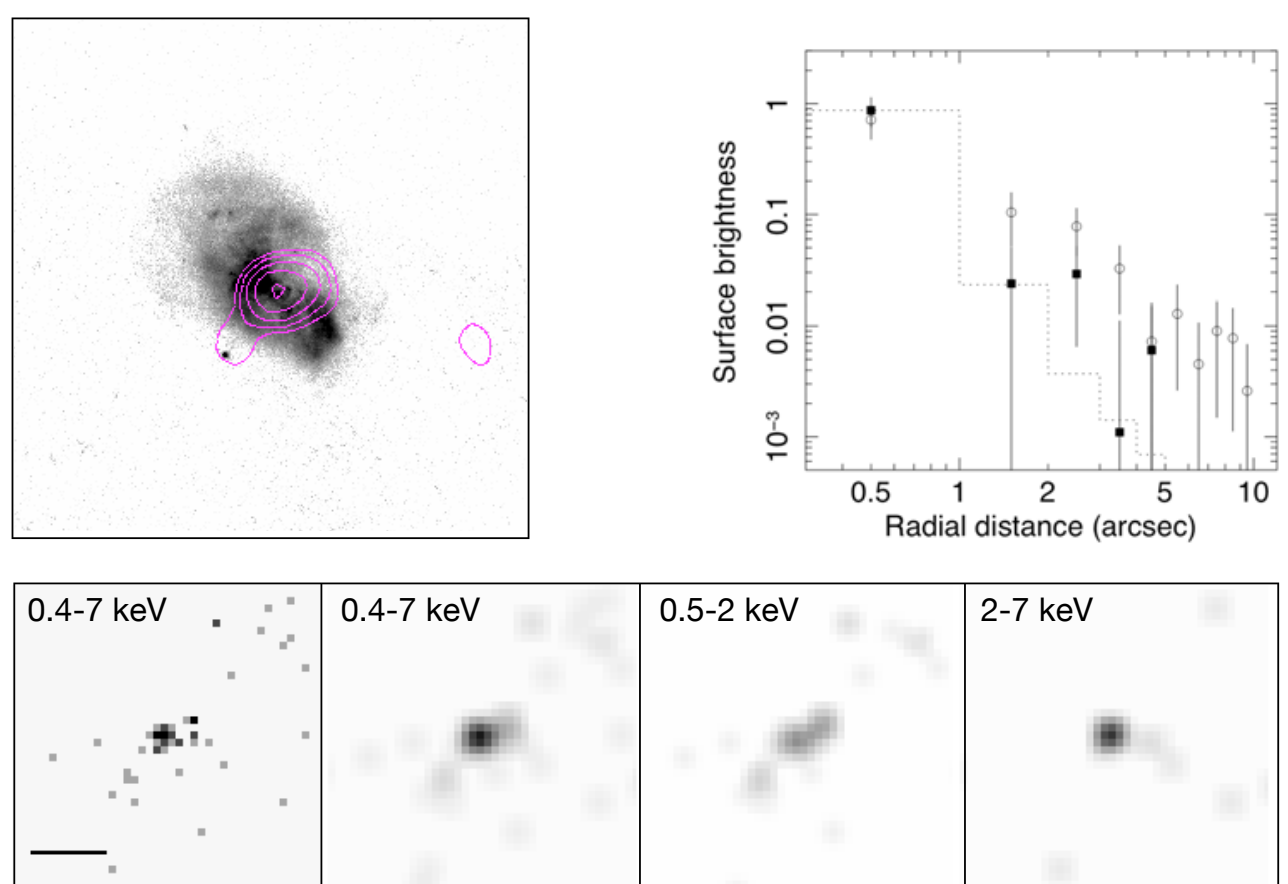

Fig. C.1. X-ray images and the surface brightness distributions for IRAS F01364-1042. An example of figure in the same format is shown in Fig. 3 of the main text with detailed description of the panels in the caption.

\section{ESO 203-IG1}

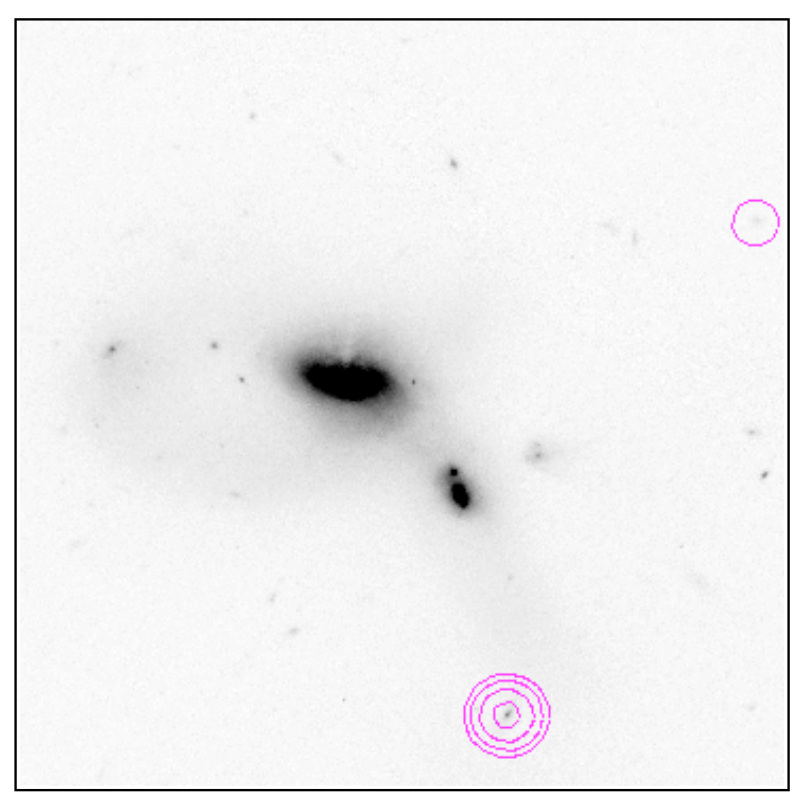

Fig. C.2. 


\section{Zw 31}
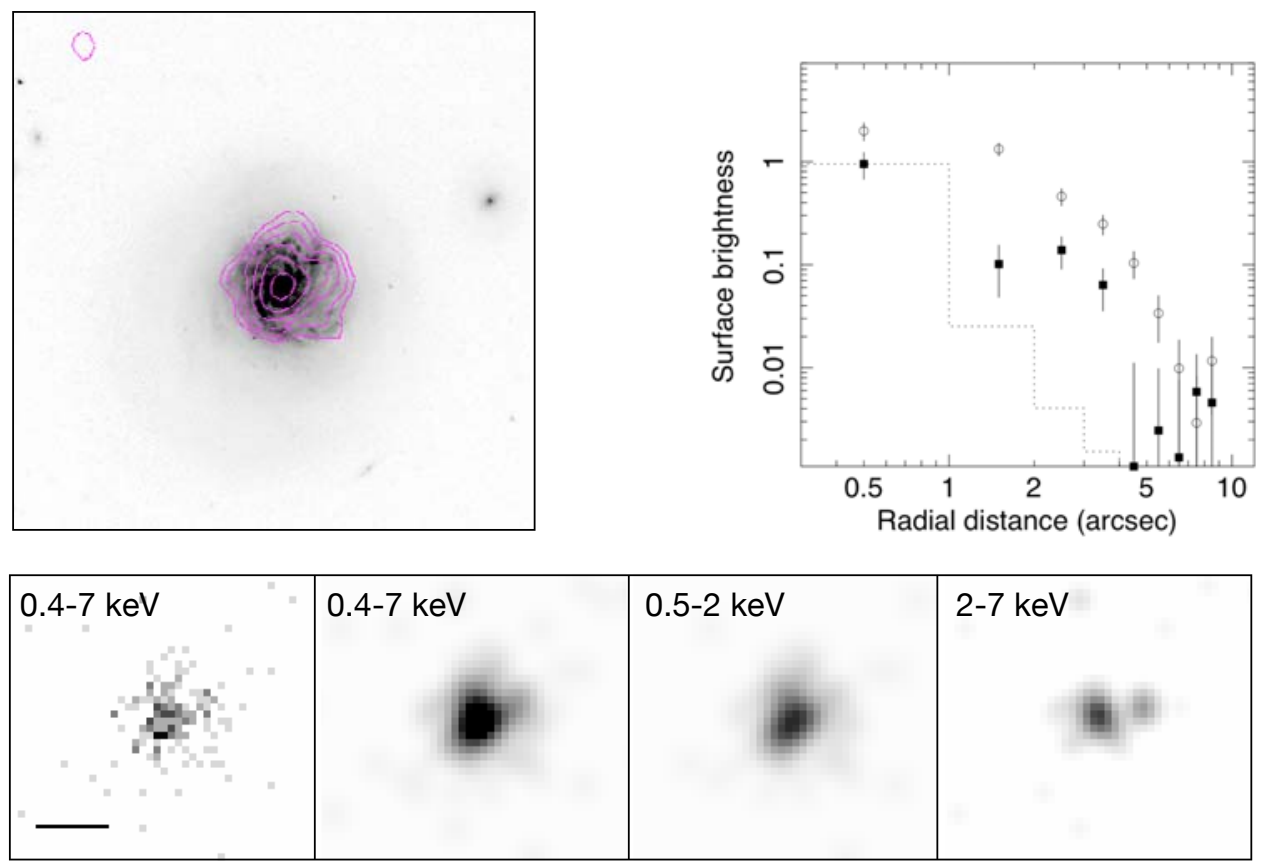

Fig. C.3.

\section{F05189-2524}
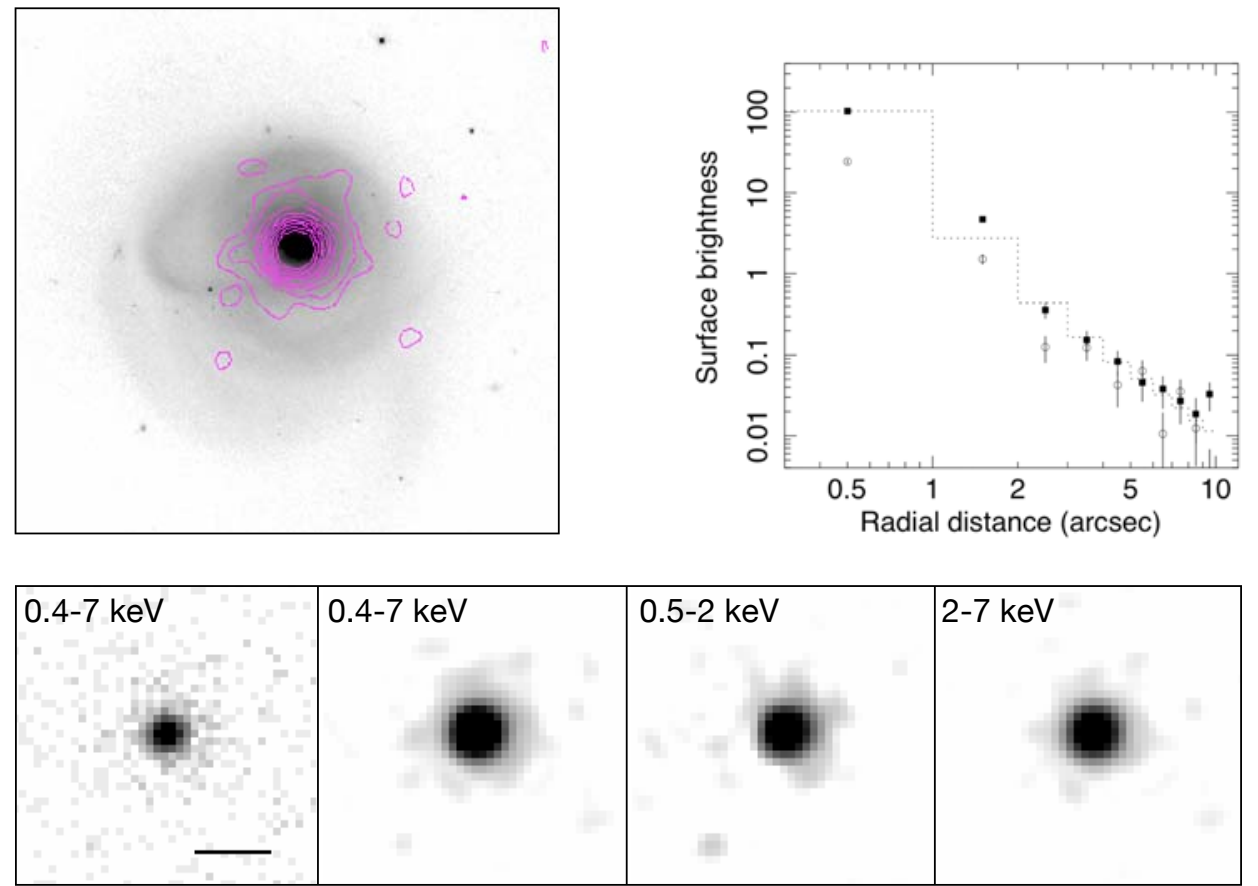

Fig. C.4. 


\section{ESO 255-IG7}
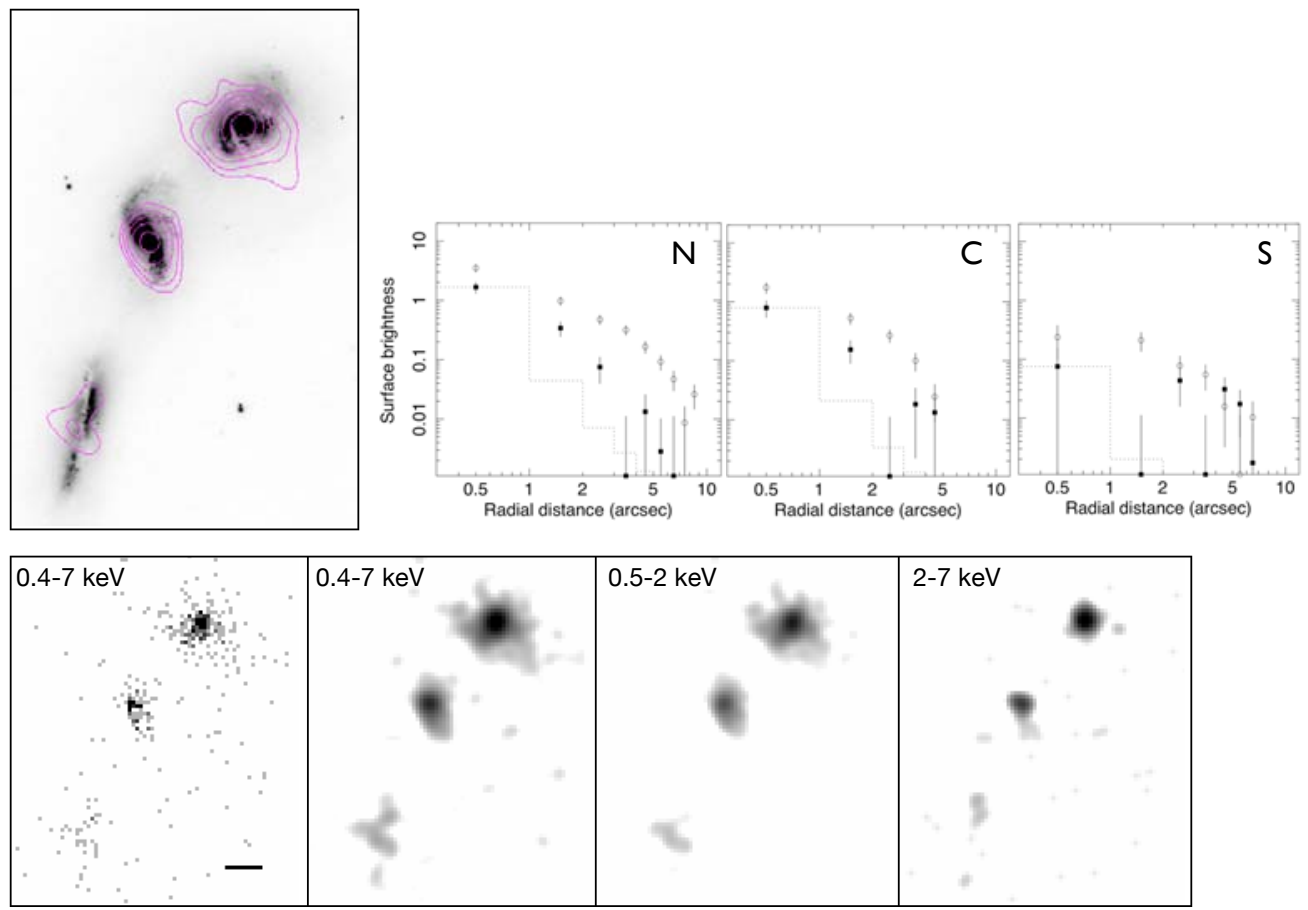

Fig. C.5.

\section{IRAS 07251-0248}
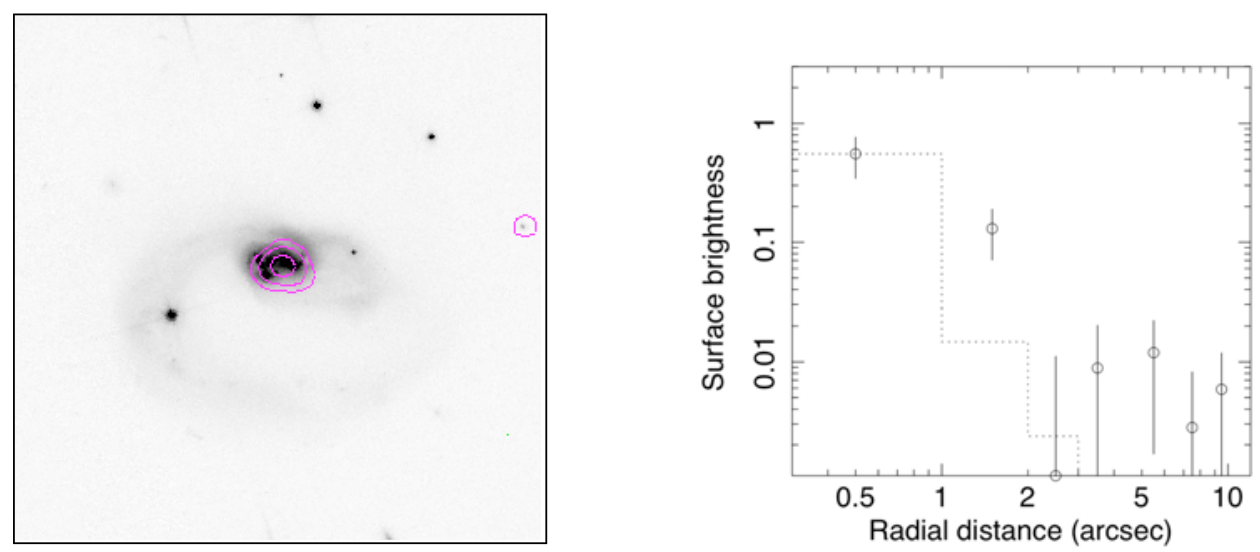

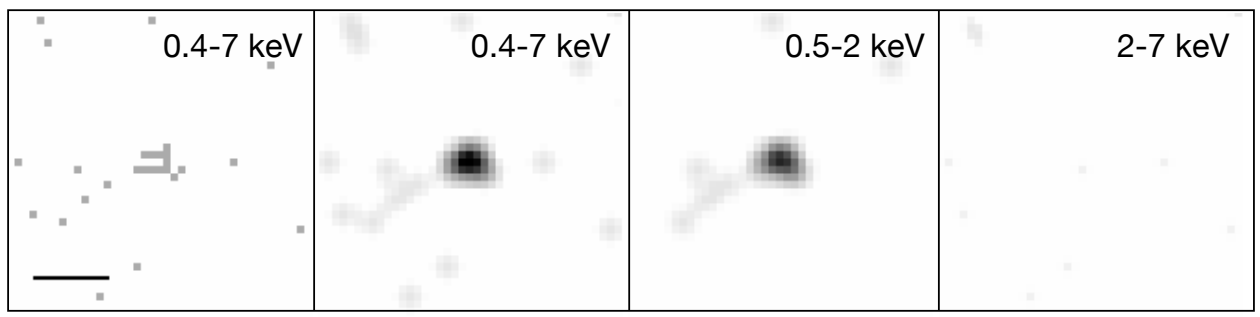

Fig. C.6. 


\section{ESO 60-IG16}
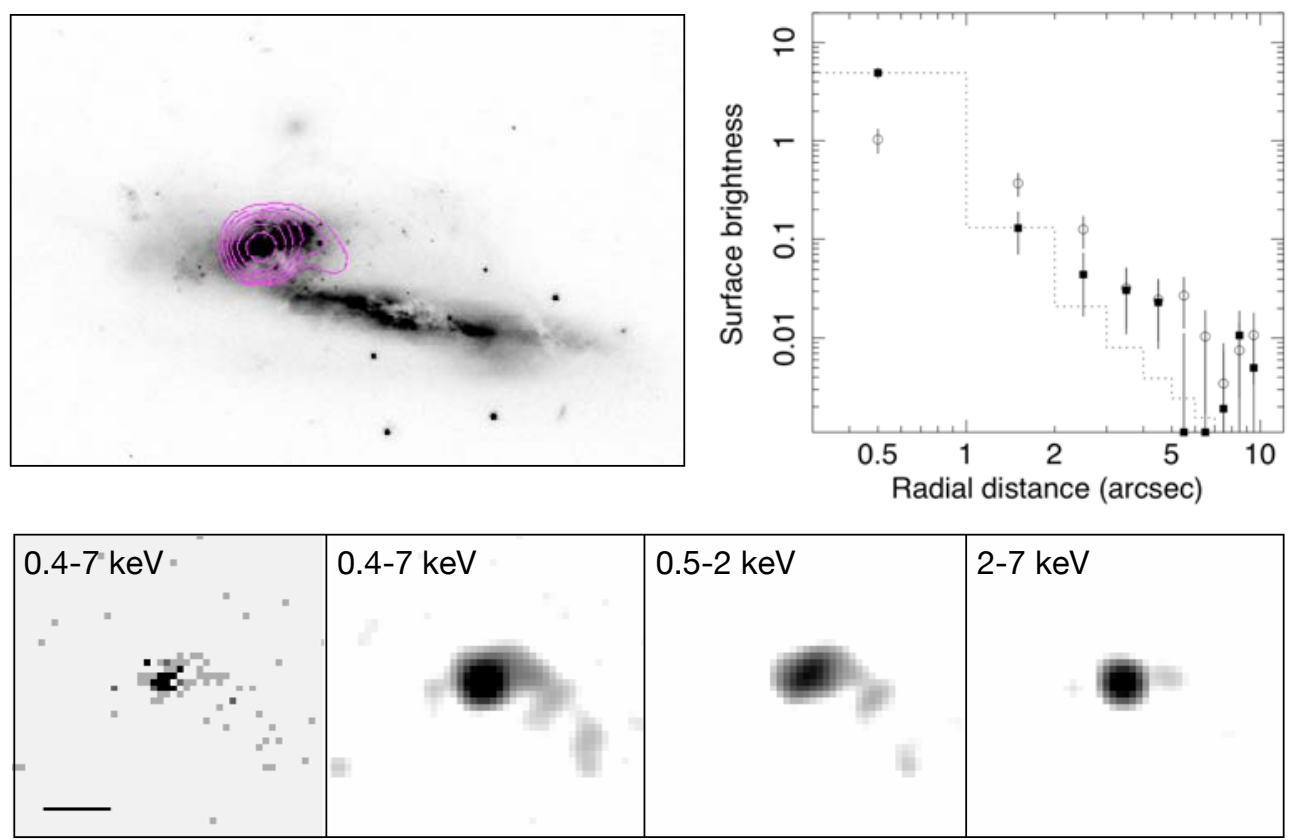

Fig. C.7.

\section{F08572+3915}

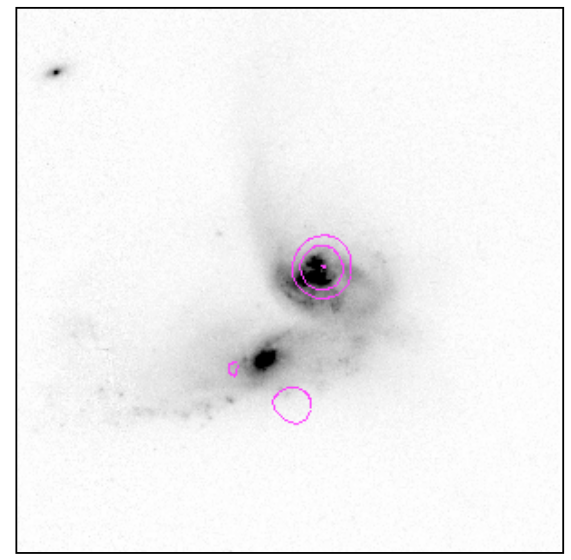

\begin{tabular}{|c|l|l|l|}
\hline $0.4-7 \mathrm{keV}$ & $0.4-7 \mathrm{keV}$ & $0.5-2 \mathrm{keV}$ & $2-7 \mathrm{keV}$ \\
& & & \\
$-\ldots .$. & & & \\
\hline
\end{tabular}

Fig. C.8. 


\section{IRAS 09022-3615}
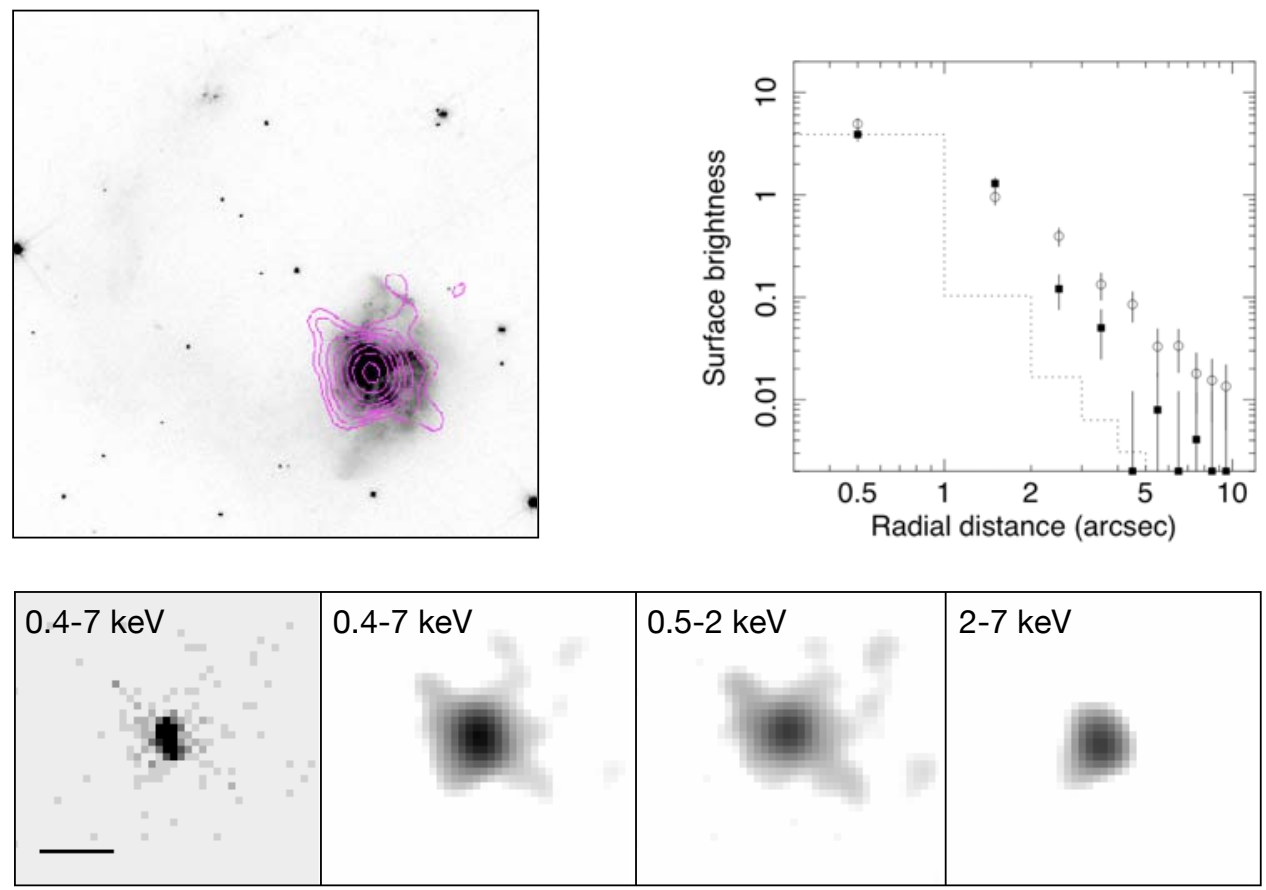

Fig. C.9.

F09111-1007
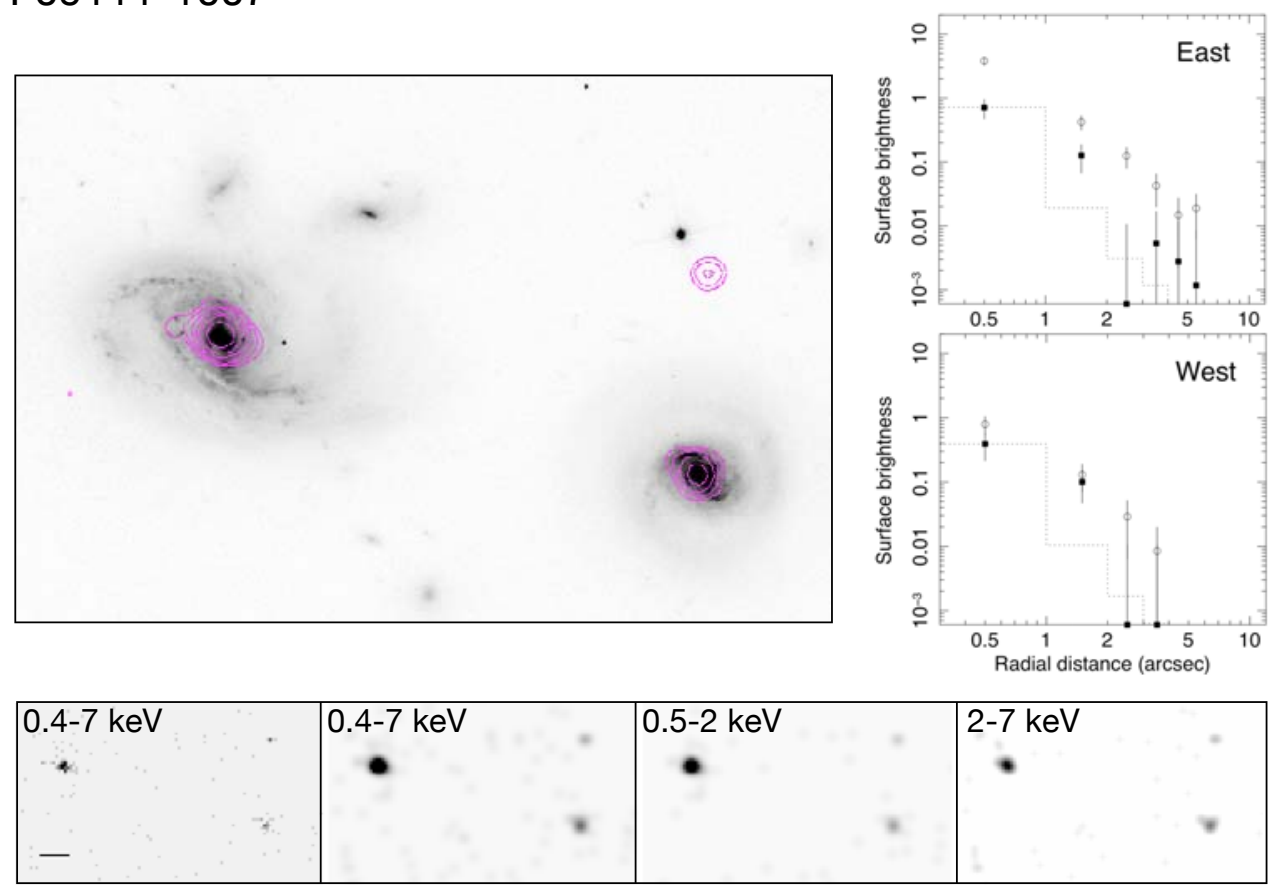

Fig. C.10. 


\section{UGC 4881}
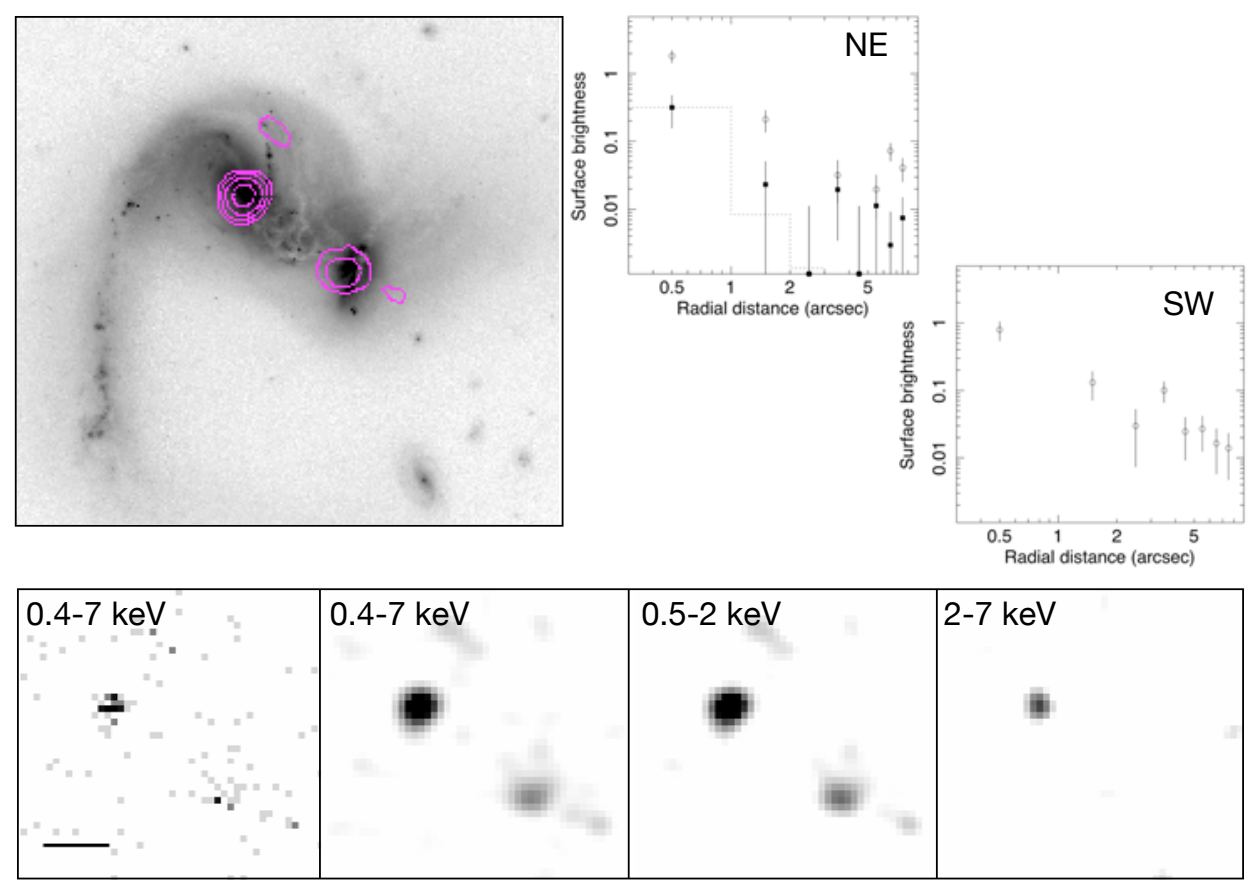

Fig. C.11.

\section{UGC 5101}
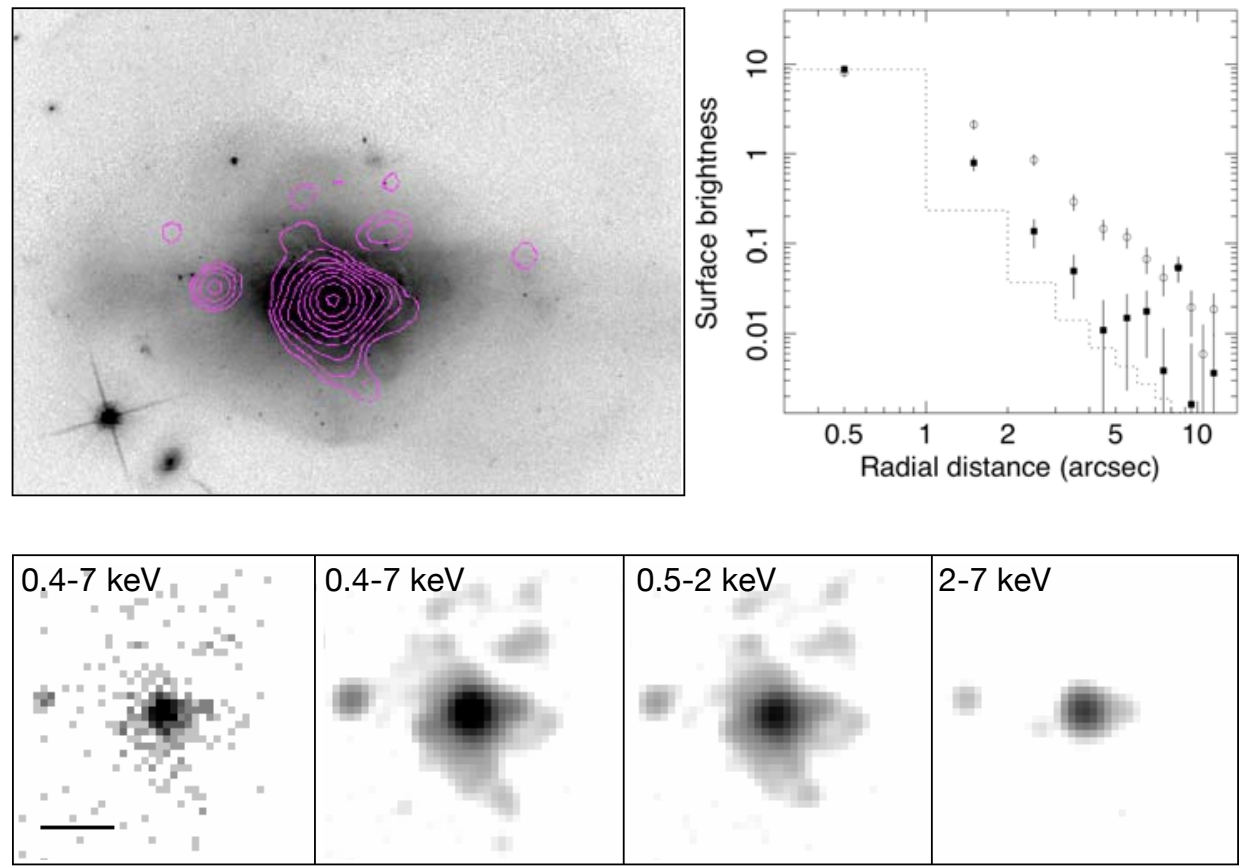

Fig. C.12. 


\section{ESO 347-IG32}
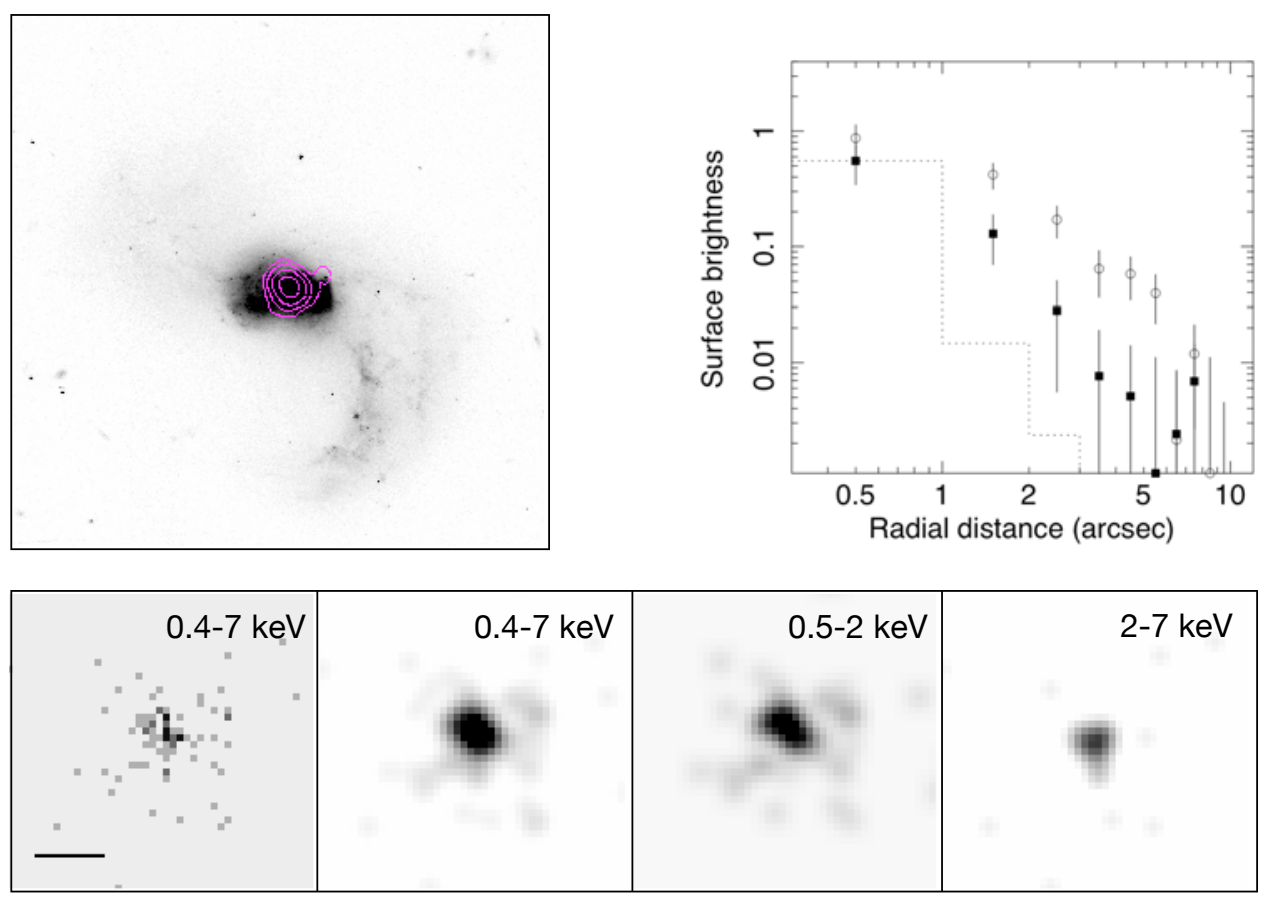

Fig. C.13.

\section{F10173+0828}

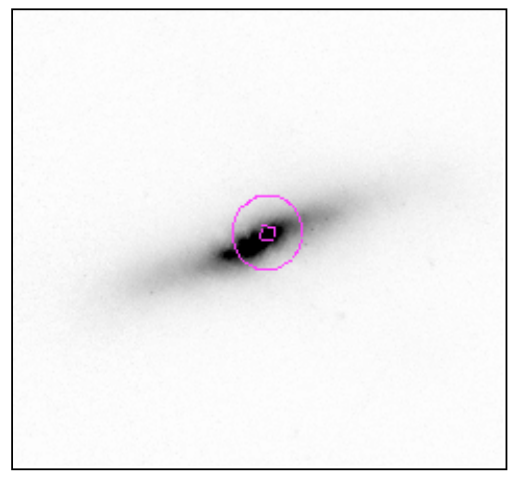

\begin{tabular}{|l|l|l|l|}
\hline $0.4-7 \mathrm{keV}$ & $0.4-7 \mathrm{keV}$ & $0.5-2 \mathrm{keV}$ & $2-7 \mathrm{keV}$ \\
& & & \\
& & & \\
\hline
\end{tabular}

Fig. C.14. 


\section{$F 10565+2448$}
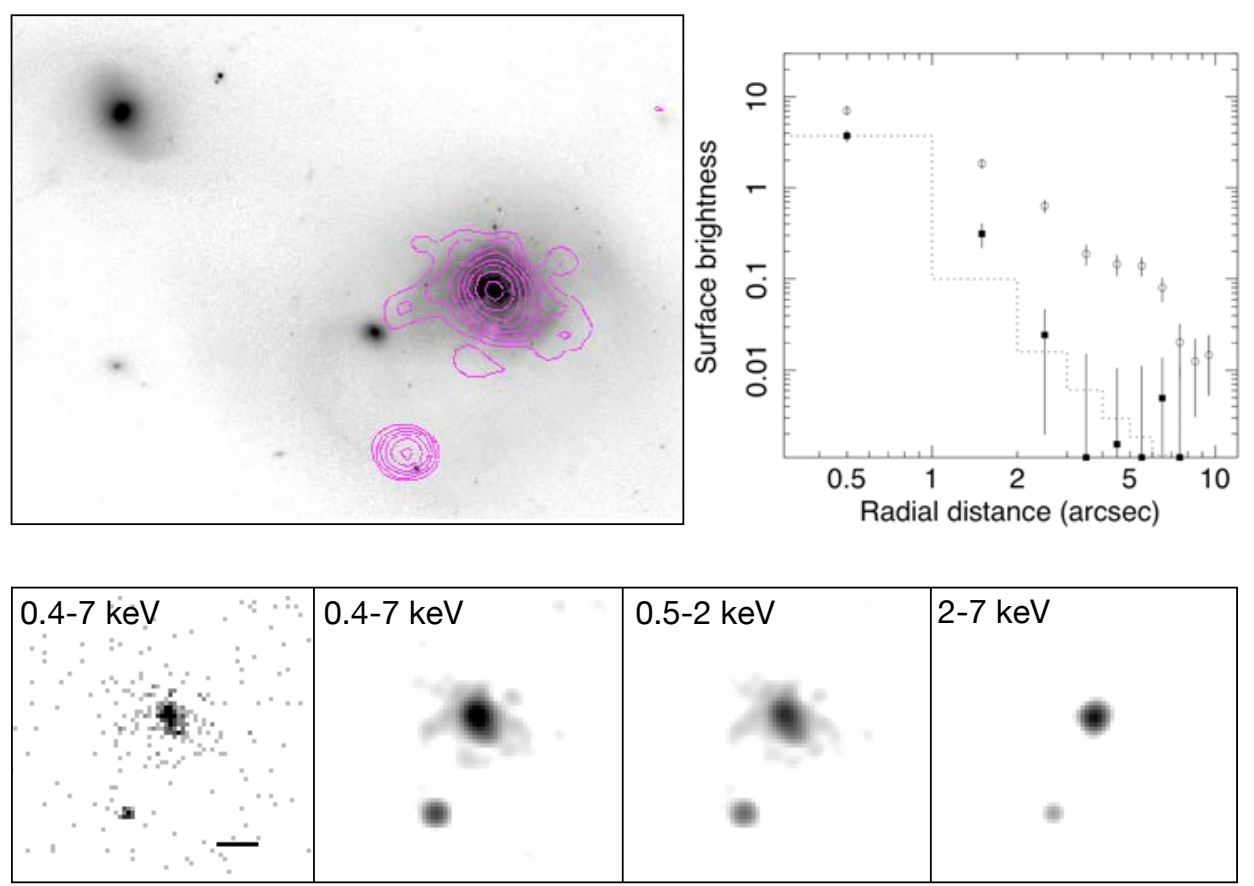

Fig. C.15.

NGC 3690
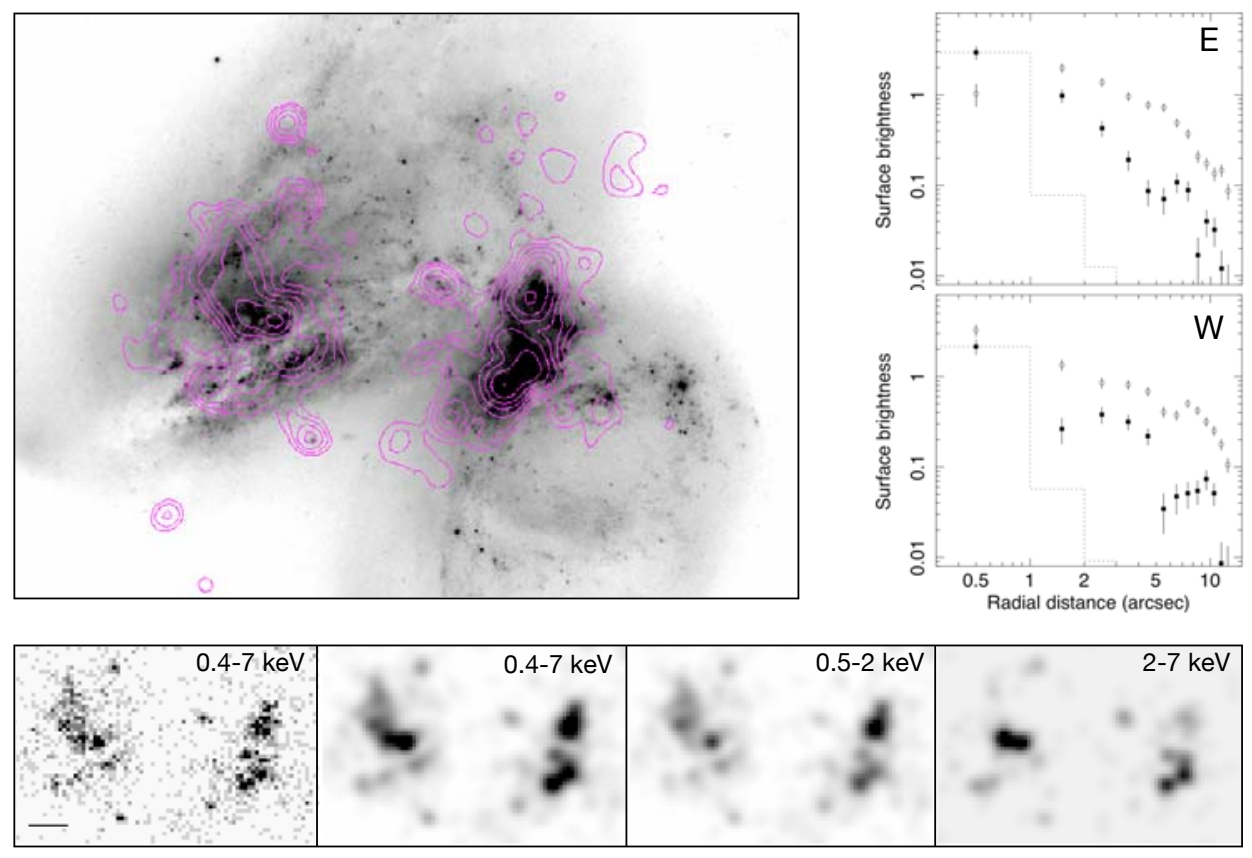

Fig. C.16. 


\section{F12112+0305}
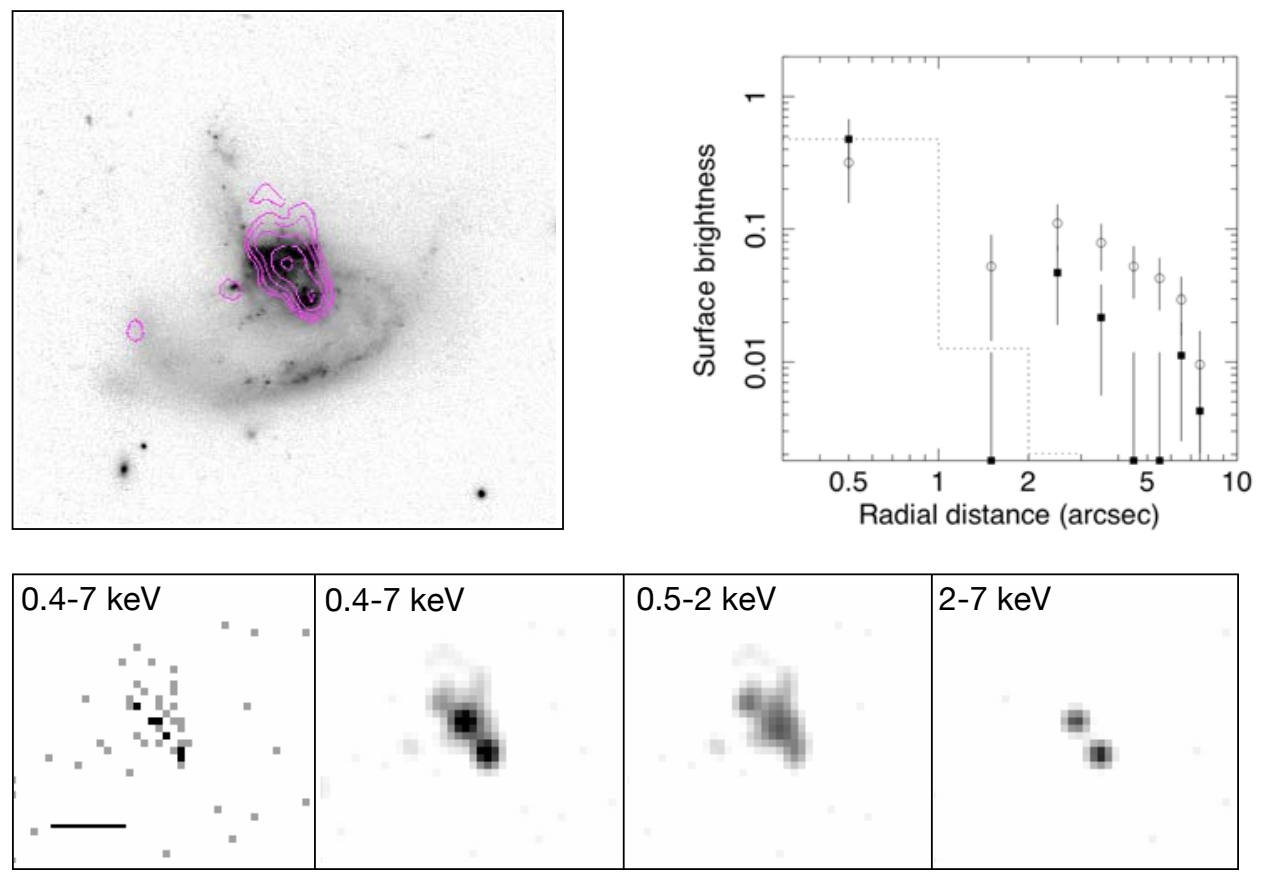

Fig. C.17.

\section{UGC 8058}
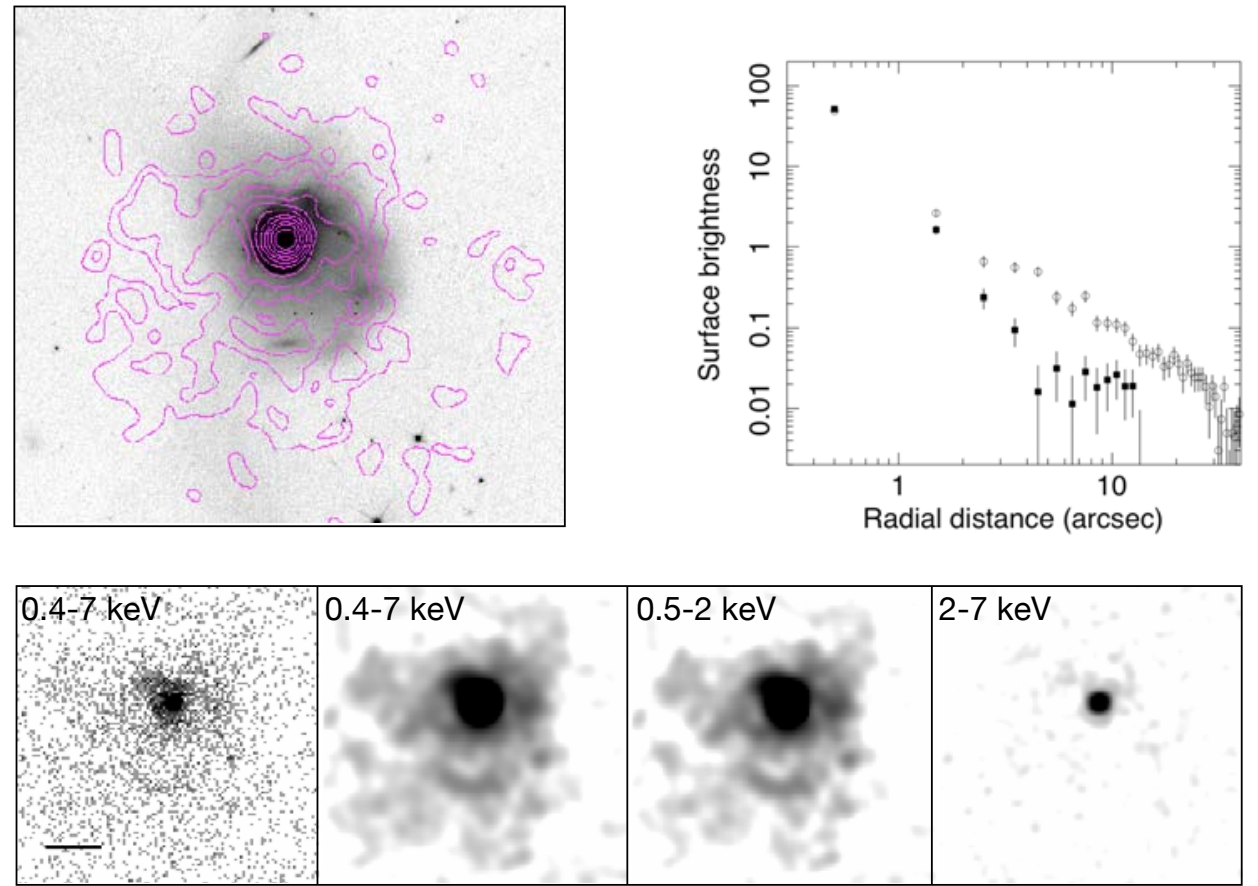

Fig. C.18. 
IRAS 13120-5453
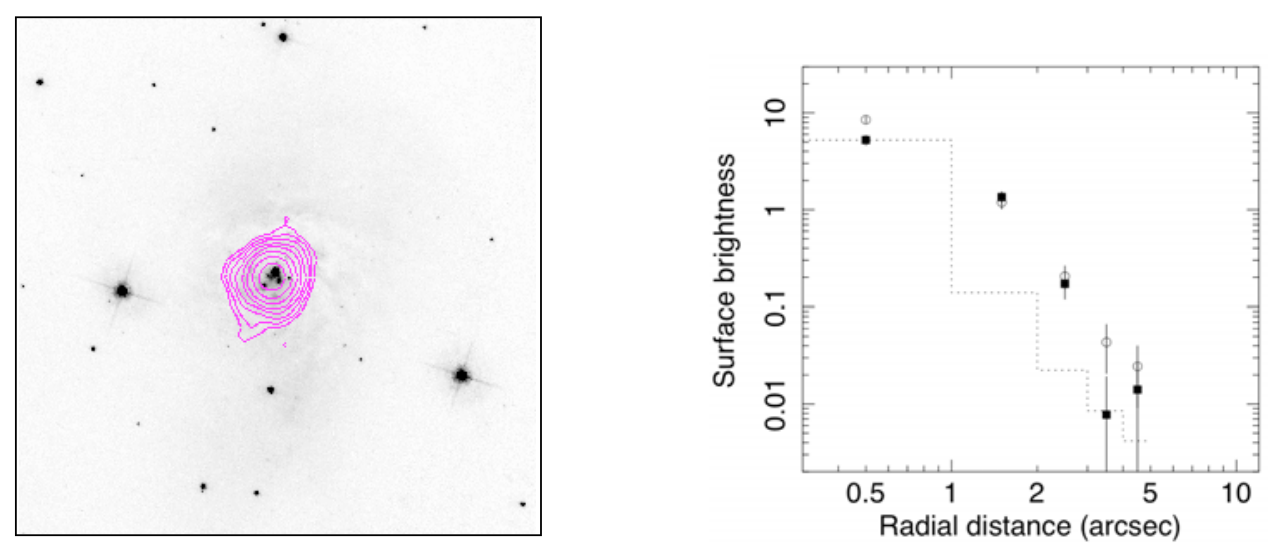

Fig. C.19.

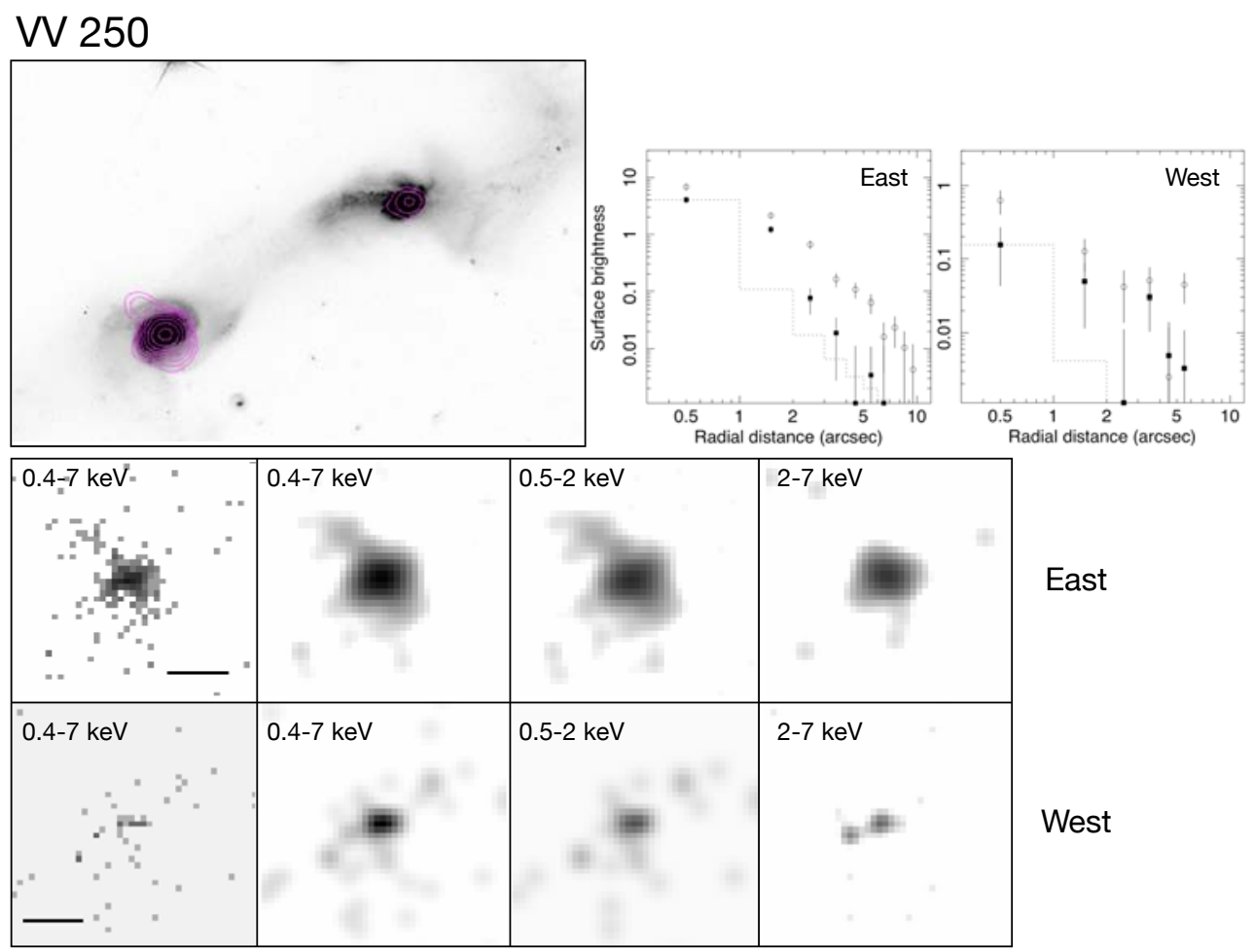

Fig. C.20. 
UGC 8387
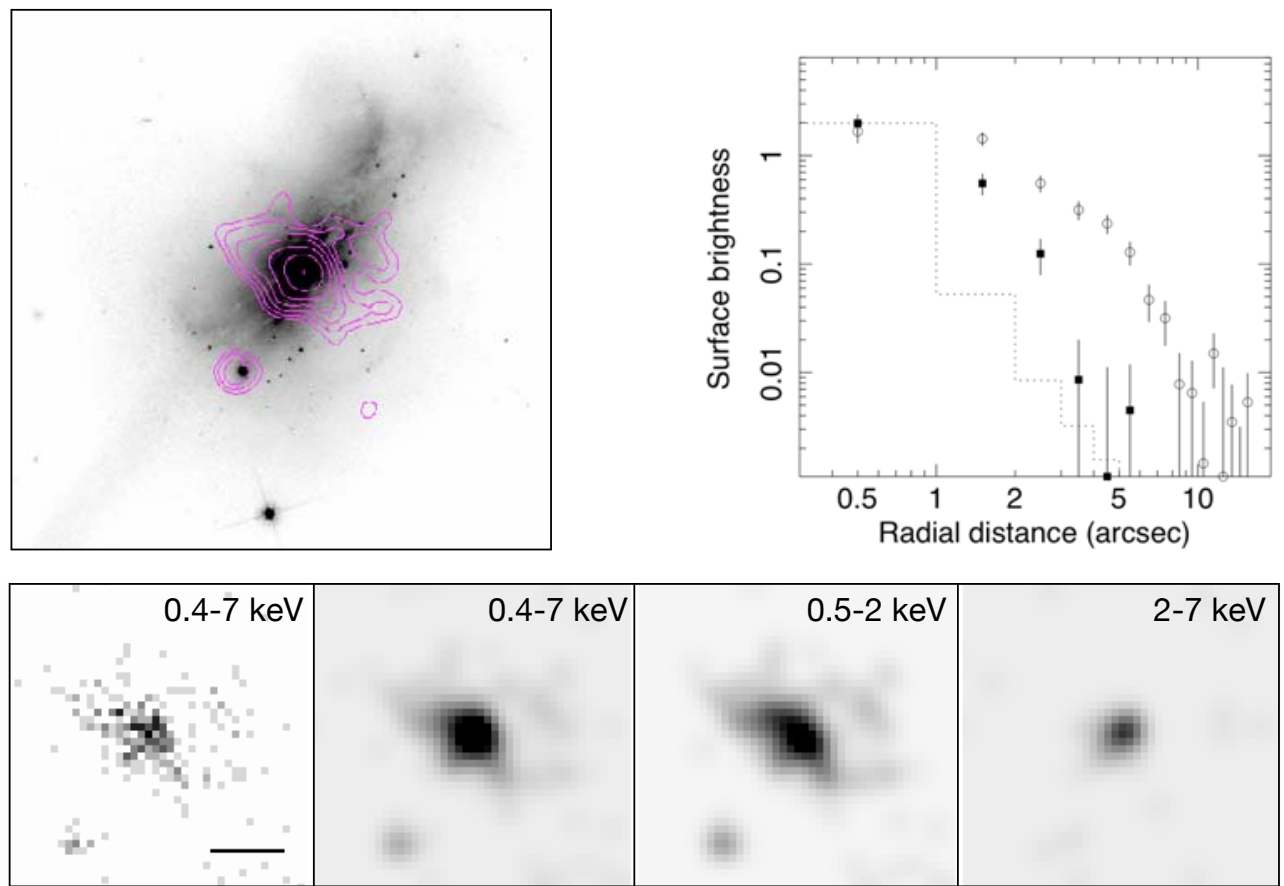

Fig. C.21.

\section{UGC 8696}
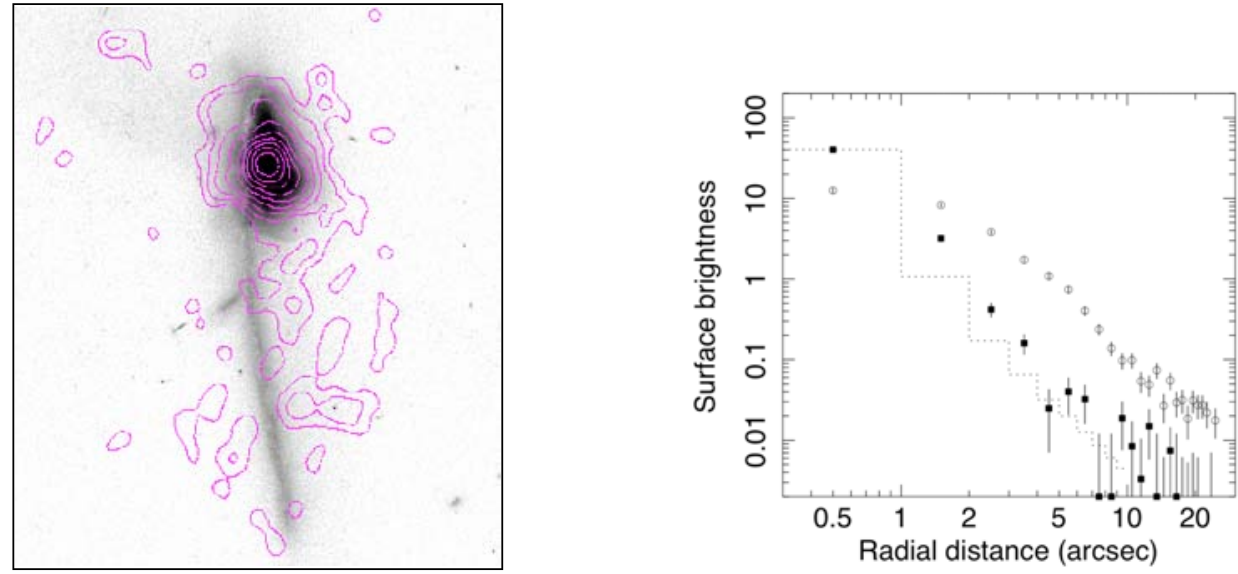

\begin{tabular}{|c|c|c|c|}
\hline $0.4-7 \mathrm{keV}$ & $0.4-7 \mathrm{keV}$ & $0.5-2 \mathrm{keV}$ & $2-7 \mathrm{keV}$ \\
& & & \\
\hline
\end{tabular}

Fig. C.22. 


\section{F14348-1447}
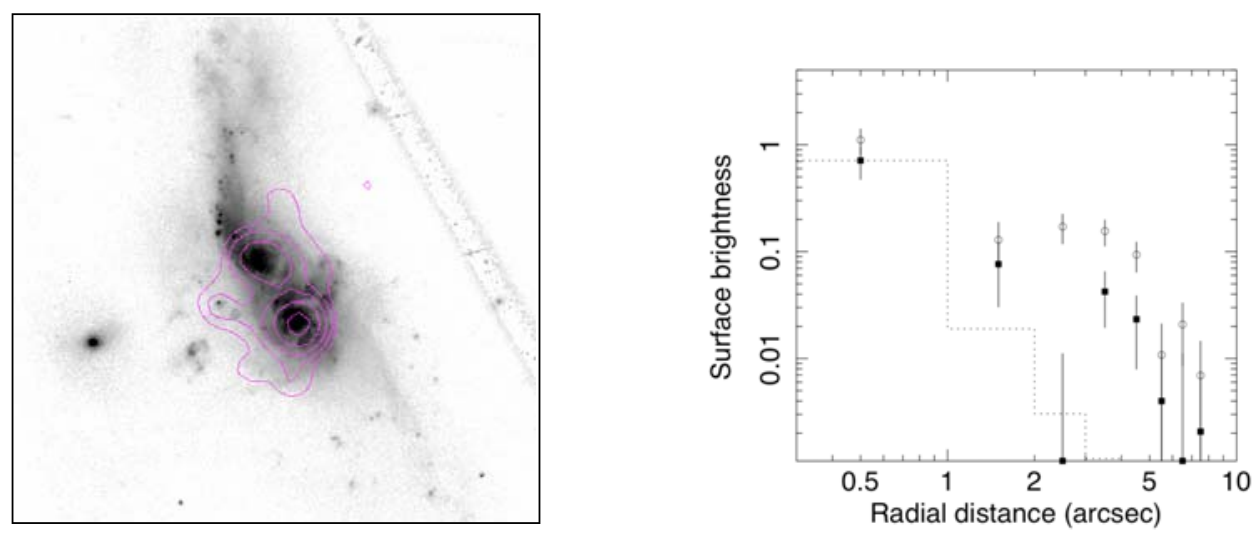

\begin{tabular}{|c|l|l|l|}
\hline $0.4-7 \mathrm{keV}$ & $0.4-7 \mathrm{keV}$ & $0.5-2 \mathrm{keV}$ & $2-7 \mathrm{keV}$ \\
\hdashline & & & \\
- & & & \\
\hline
\end{tabular}

Fig. C.23.

\section{F14378-3651}
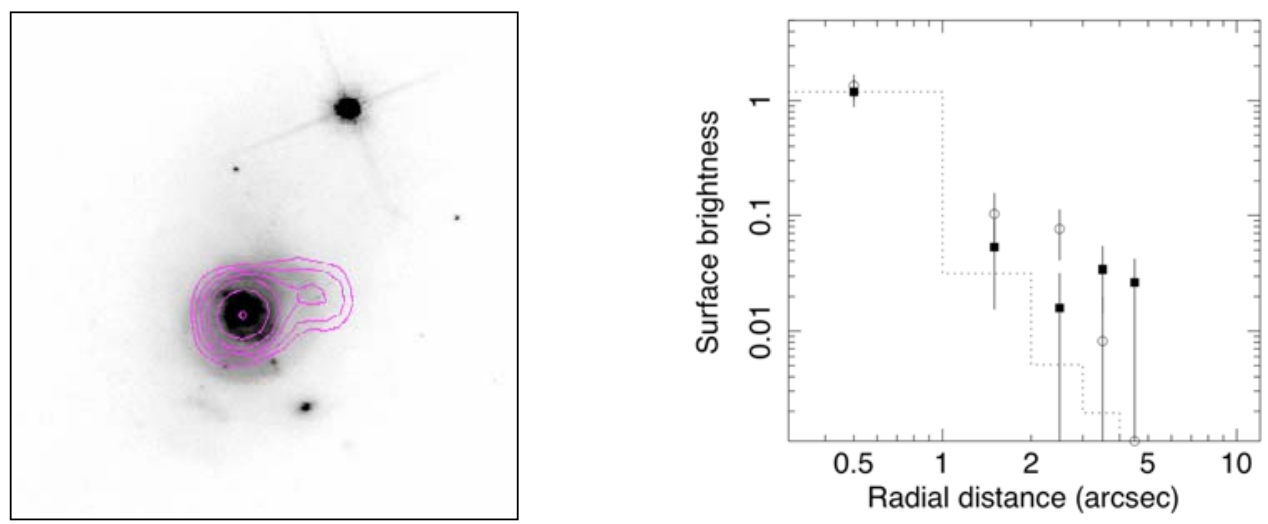

\begin{tabular}{|l|l|l|l|}
\hline $0.4-7 \mathrm{keV}$ & $0.4-7 \mathrm{keV}$ & $0.5-2 \mathrm{keV}$ & $2-7 \mathrm{keV}$ \\
& & &
\end{tabular}

Fig. C.24. 


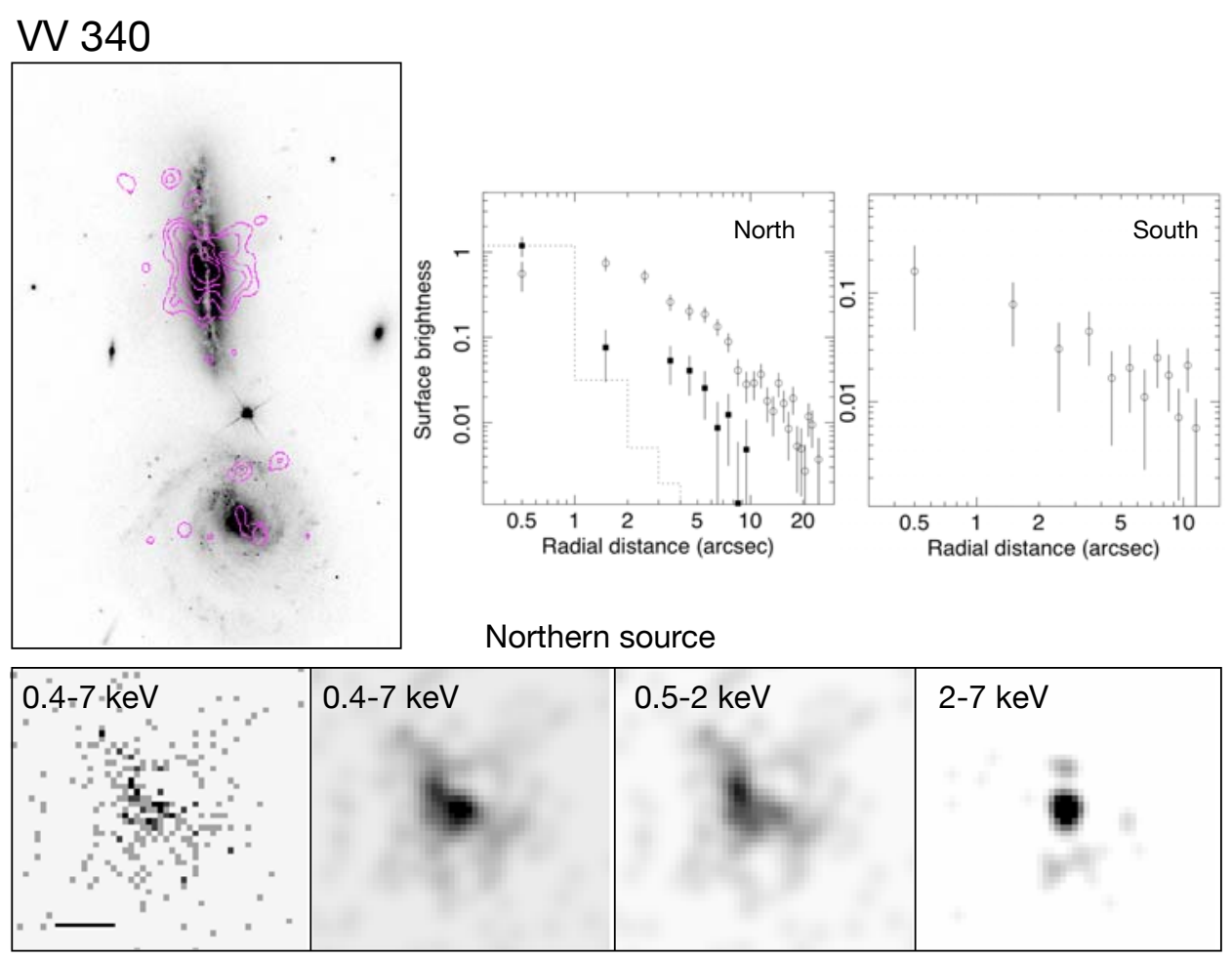

Fig. C.25.

\section{VV 705}

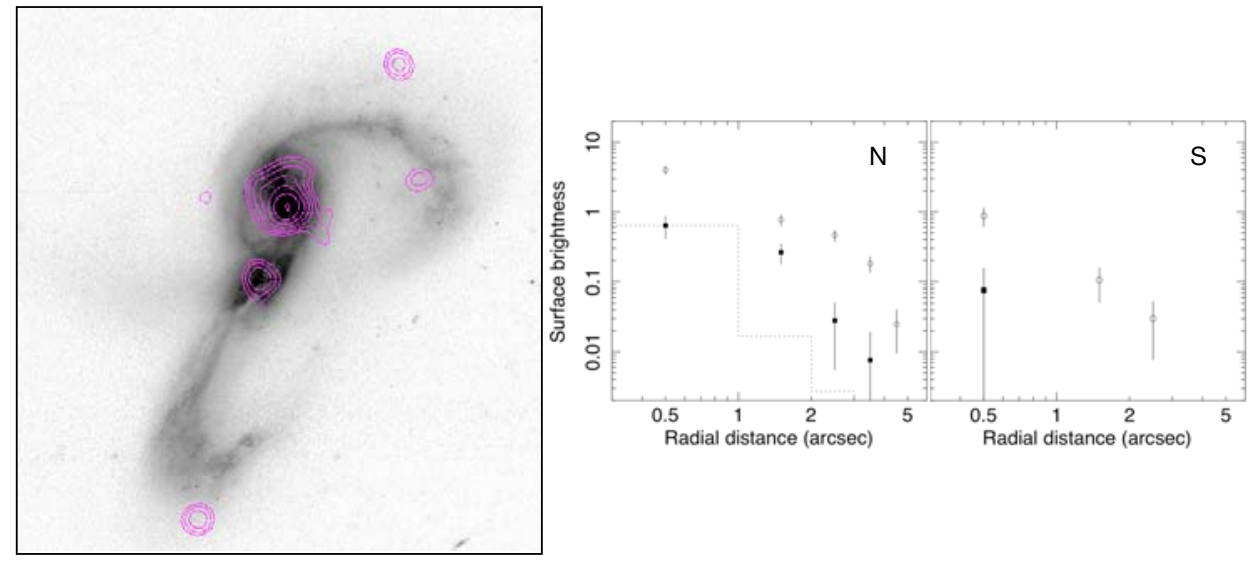

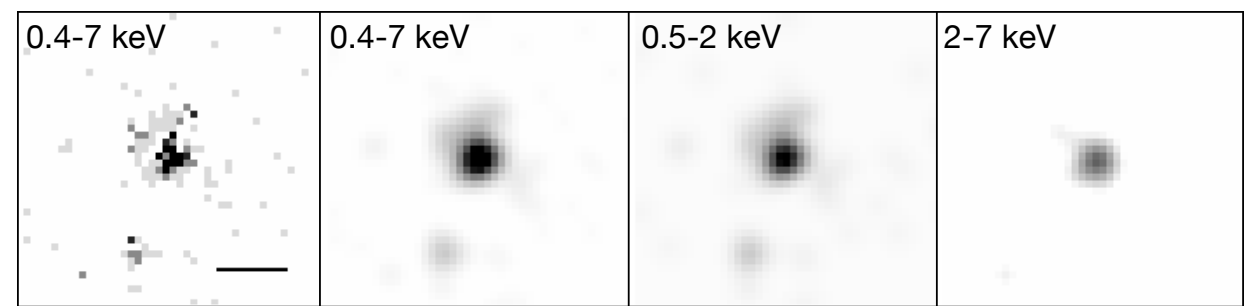

Fig. C.26. 


\section{$F 15250+3608$}
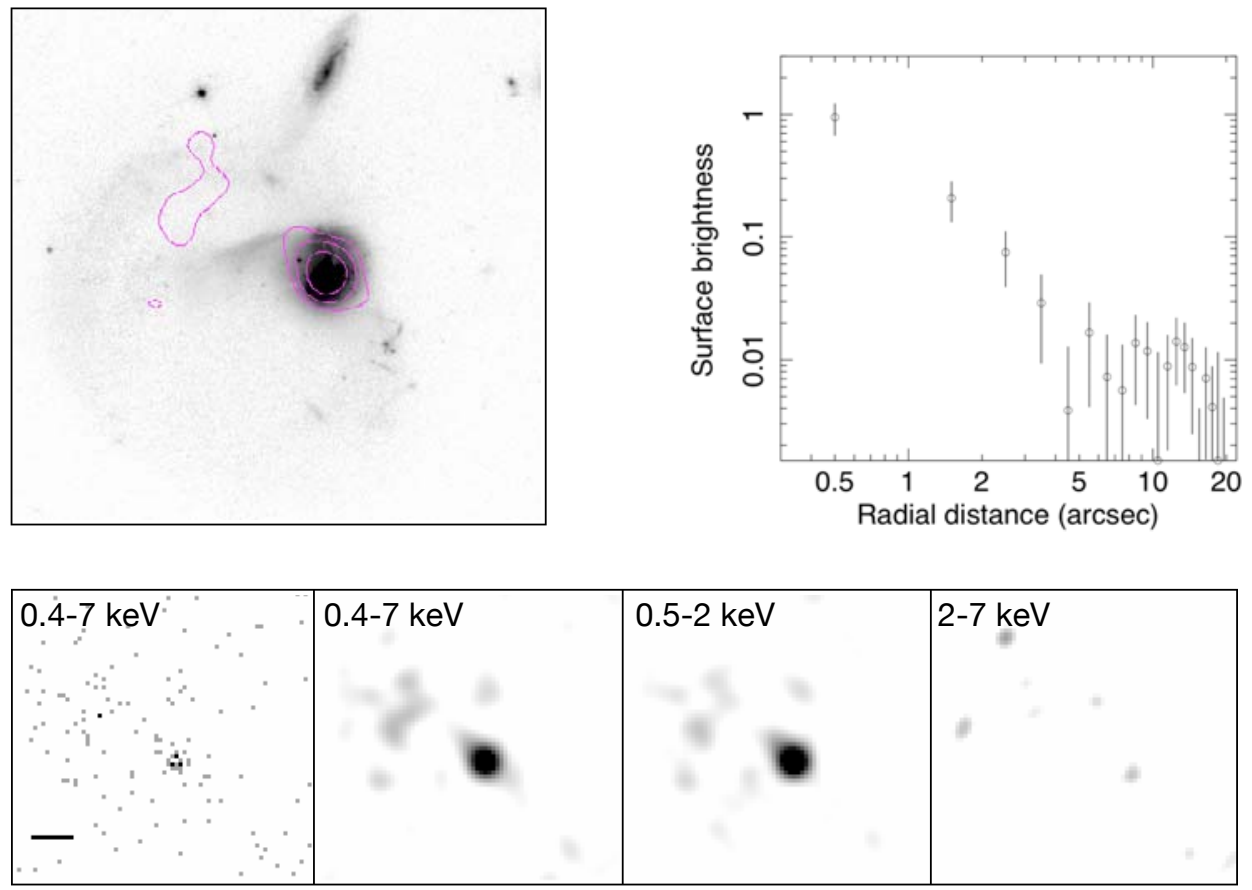

Fig. C.27.

\section{UGC 9913}
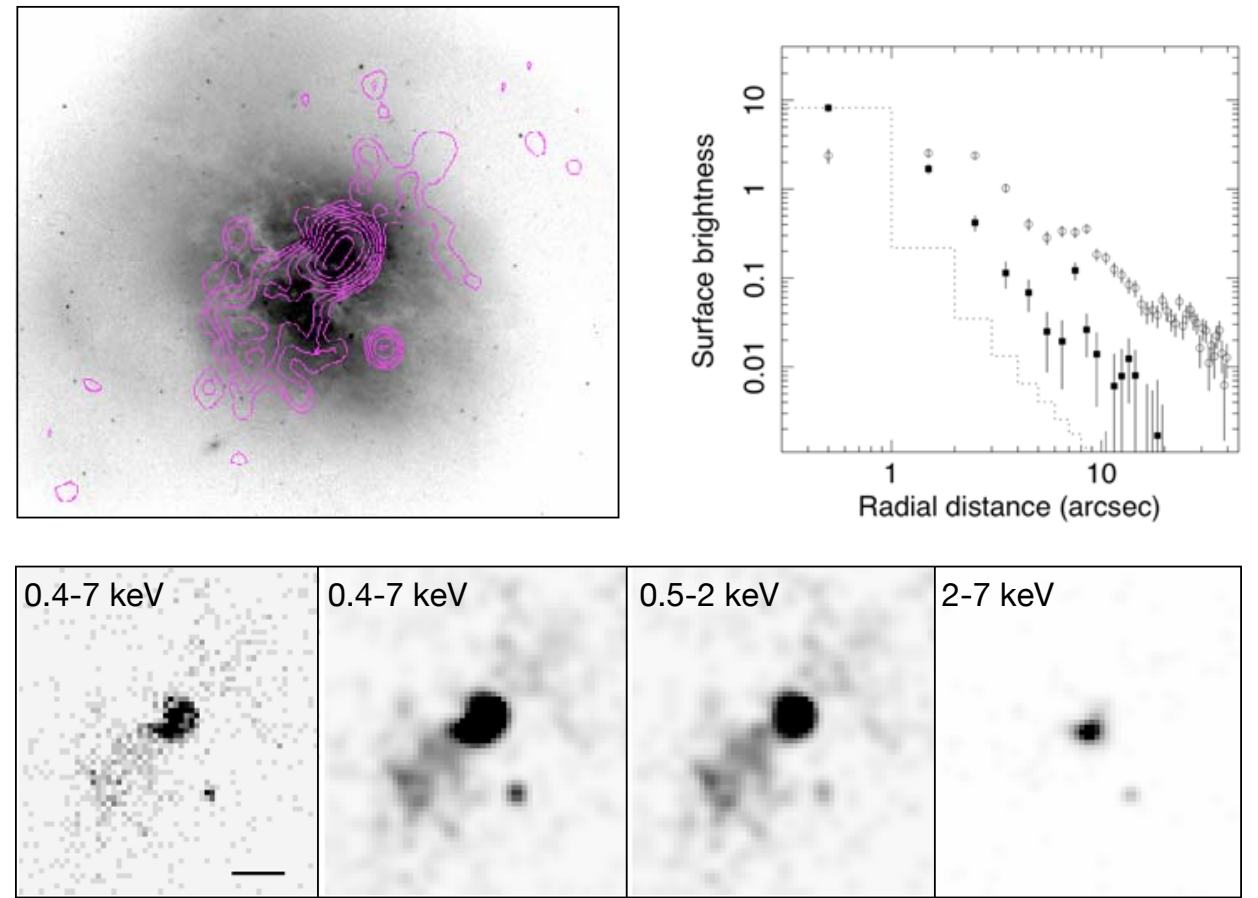

Fig. C.28. 


\section{ESO 69-IG6}
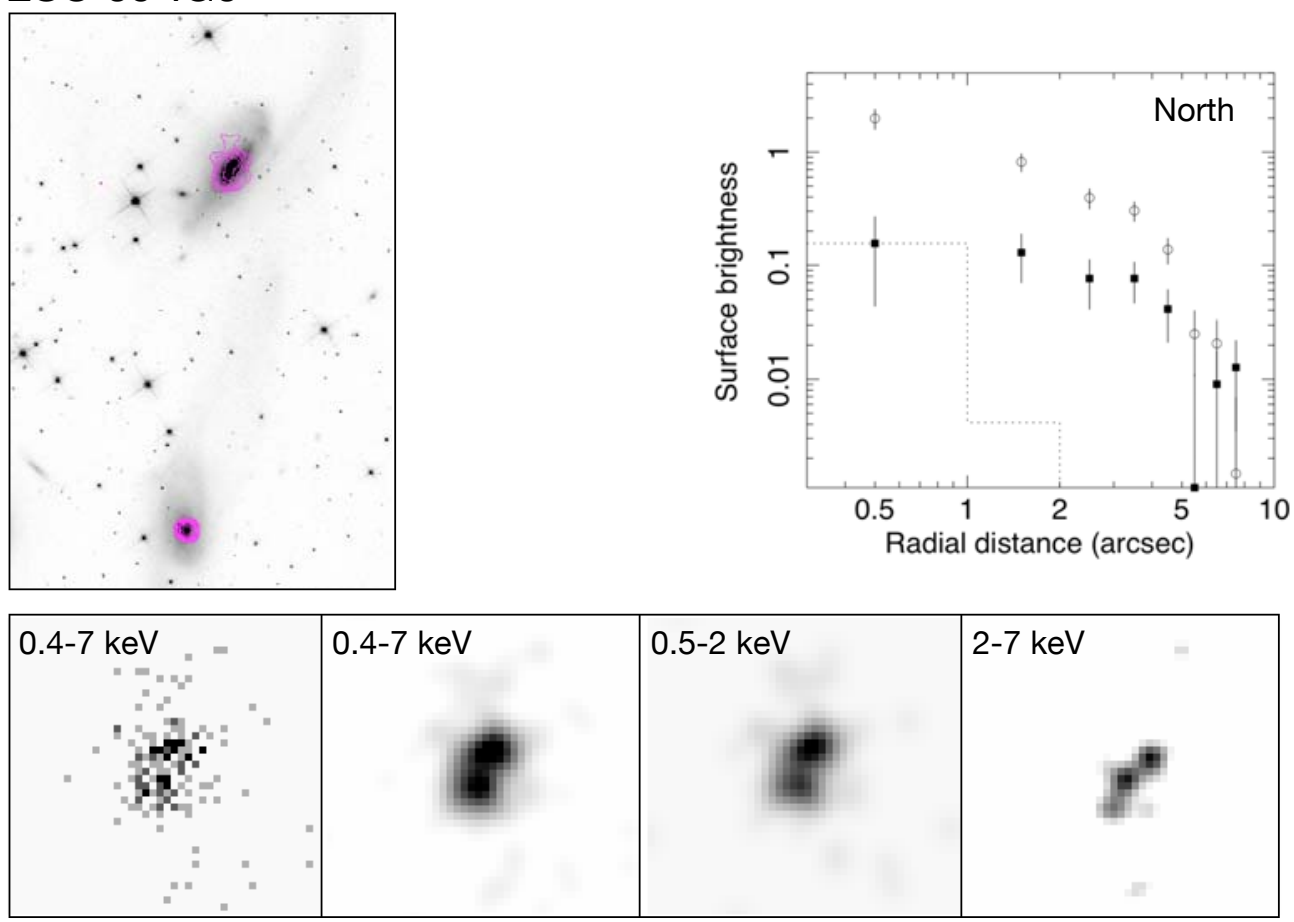

Fig. C.29.

NGC 6240
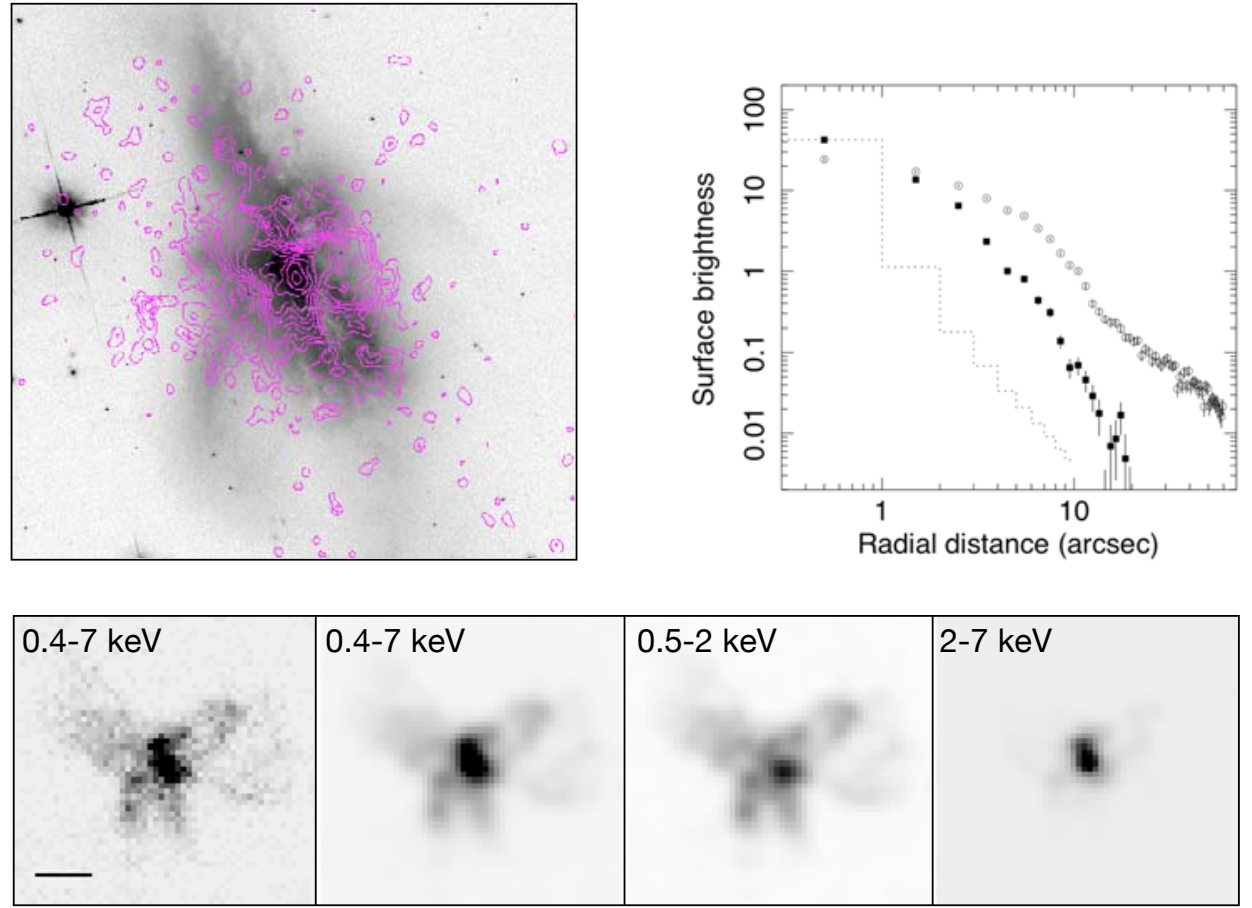

Fig. C.30. 


\section{$F 17132+5313$}
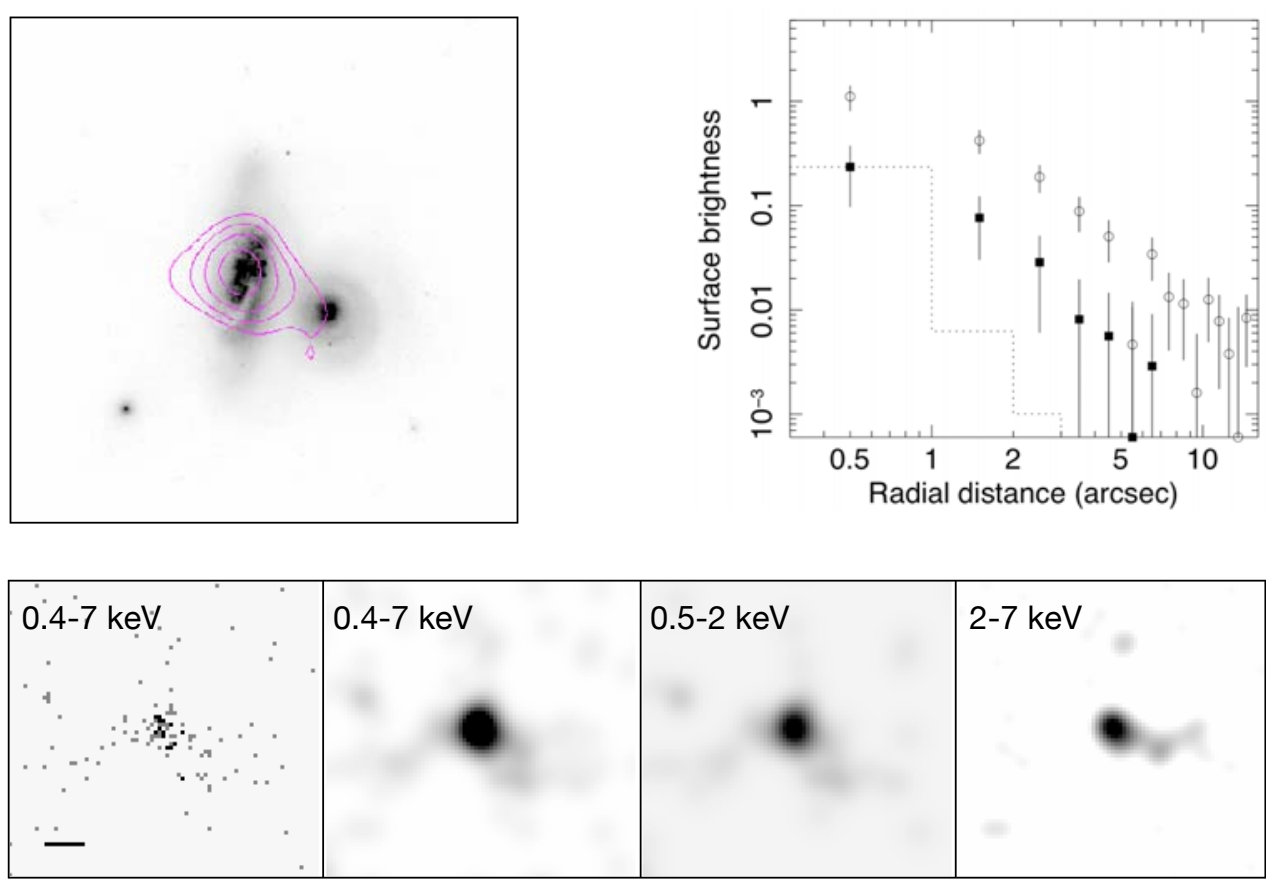

Fig. C.31.

\section{F17207-0014}
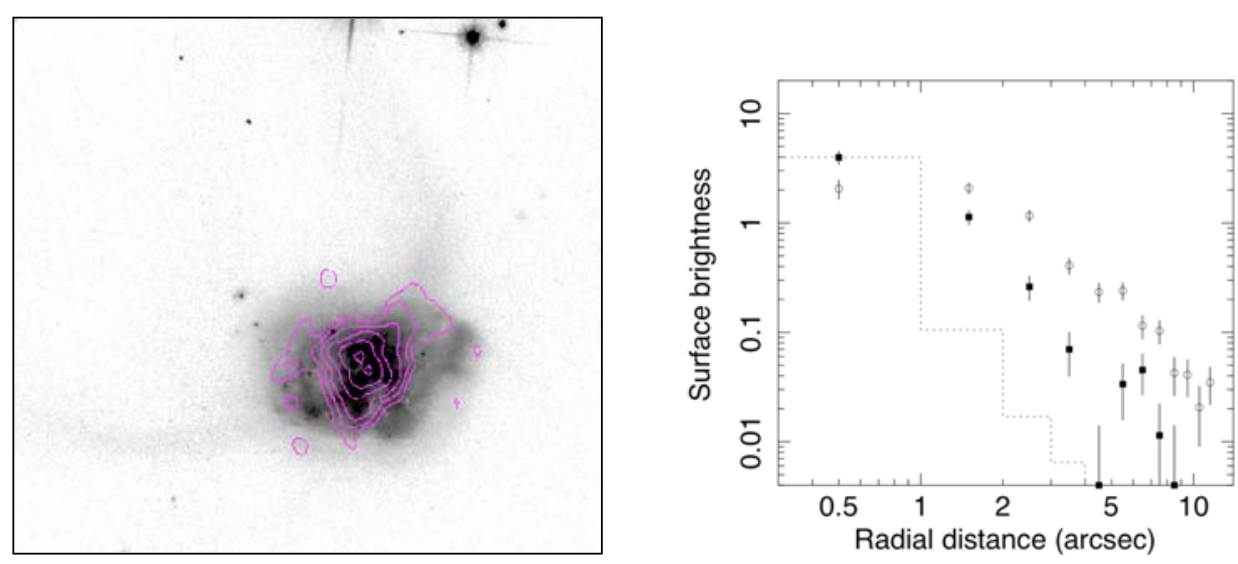

\begin{tabular}{|c|l|l|l|}
\hline $0.4-7 \mathrm{keV}$ & $0.4-7 \mathrm{keV}$ & $0.5-2 \mathrm{keV}$ & $2-7 \mathrm{keV}$ \\
$y$ & & & \\
& & &
\end{tabular}

Fig. C.32. 


\section{F18293-3413}
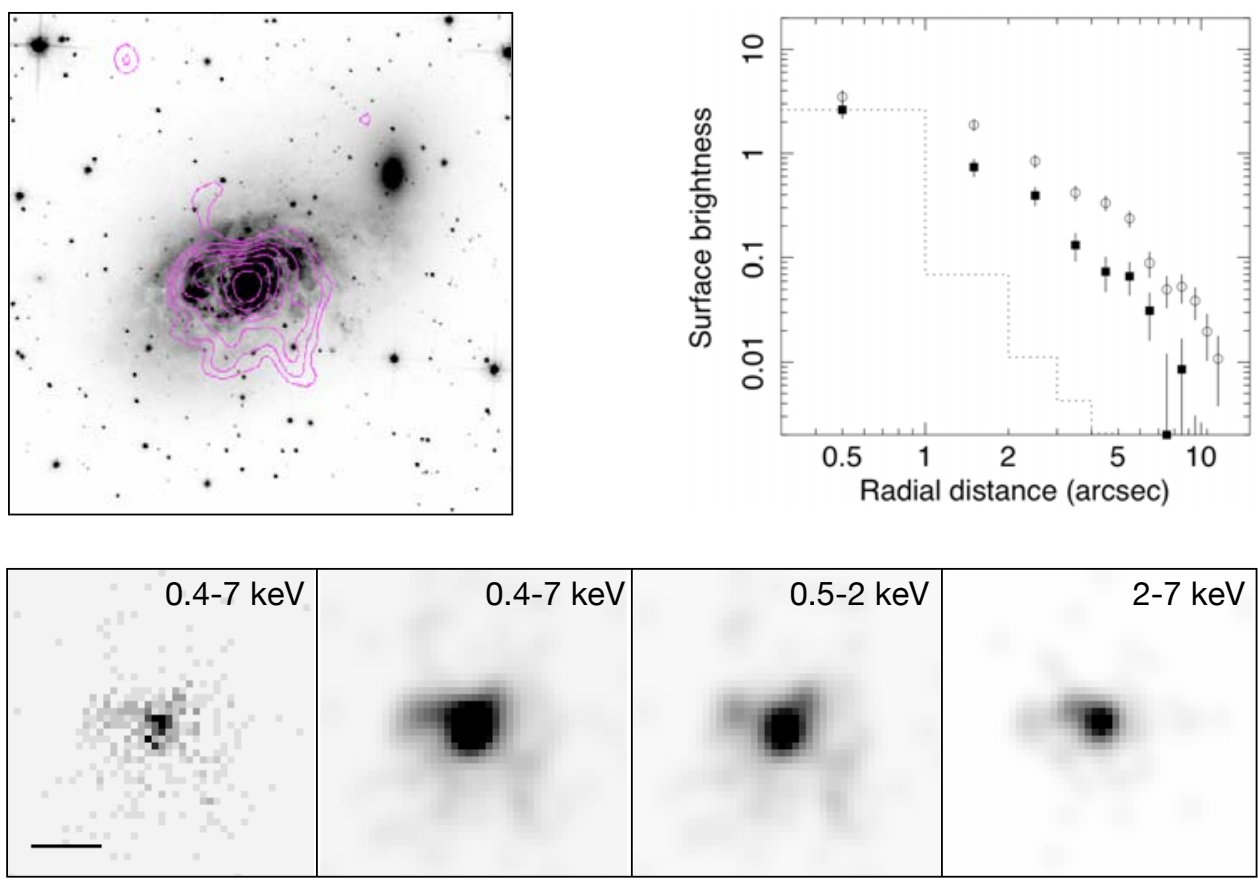

Fig. C.33.

\section{ESO 593-IG8}
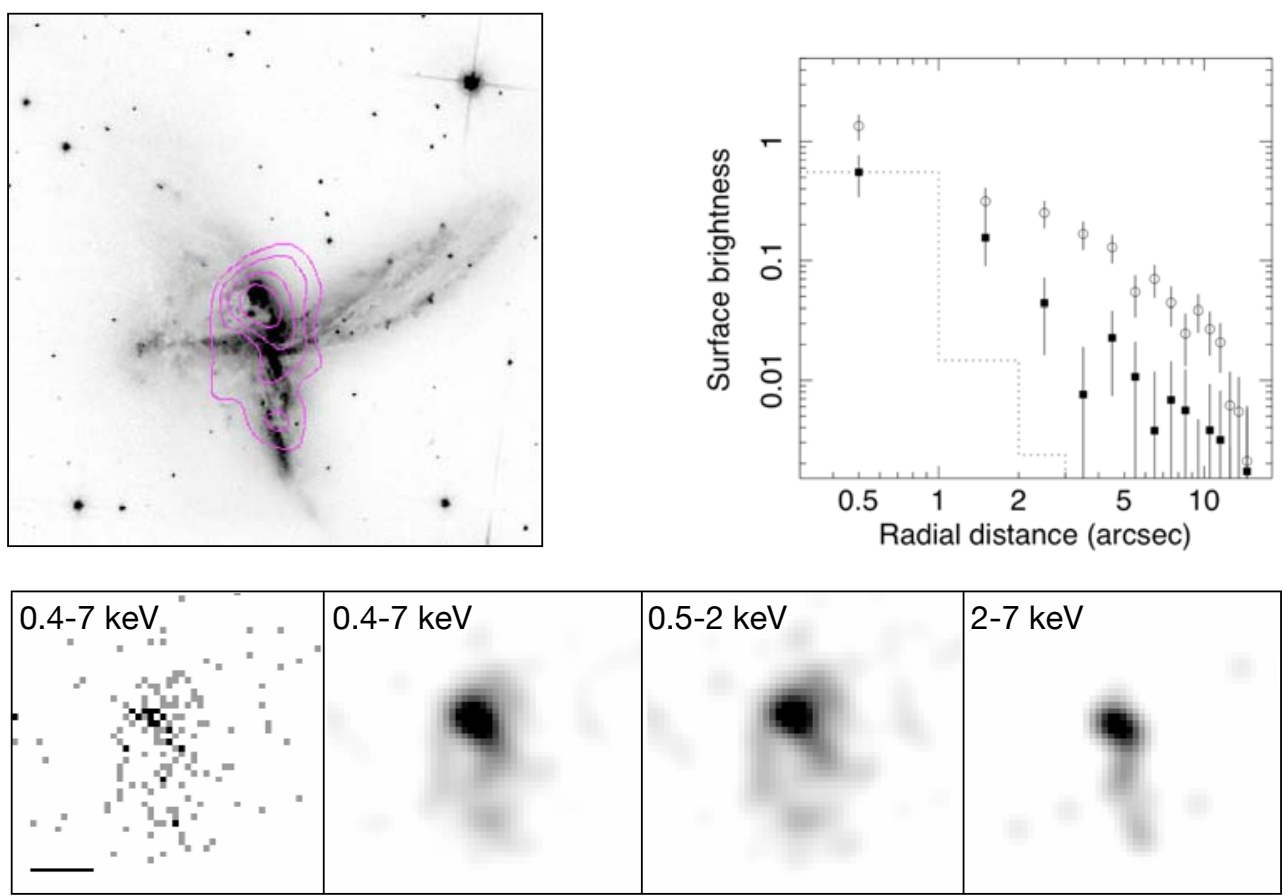

Fig. C.34. 


\section{F19297-0406}
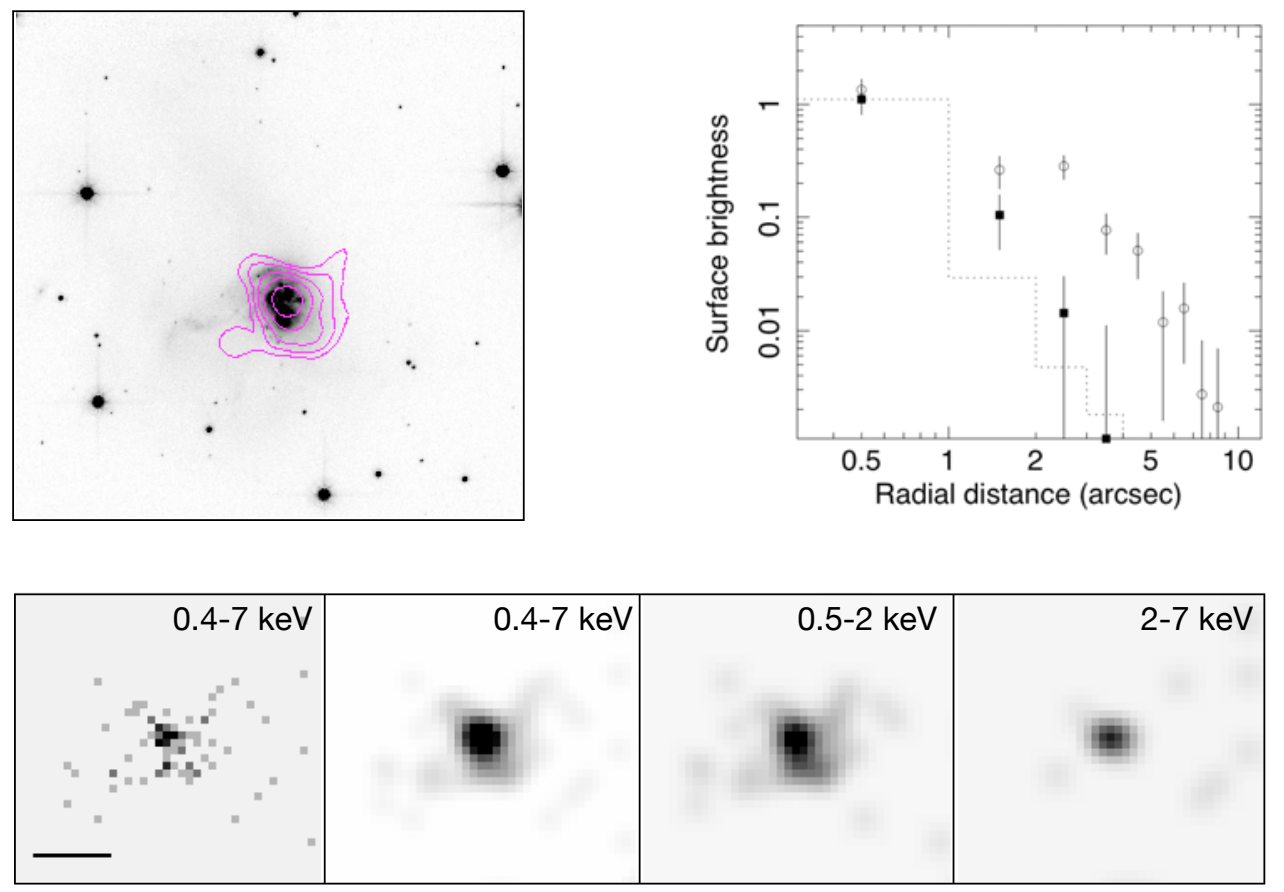

Fig. C.35.

IRAS $19542+1110$
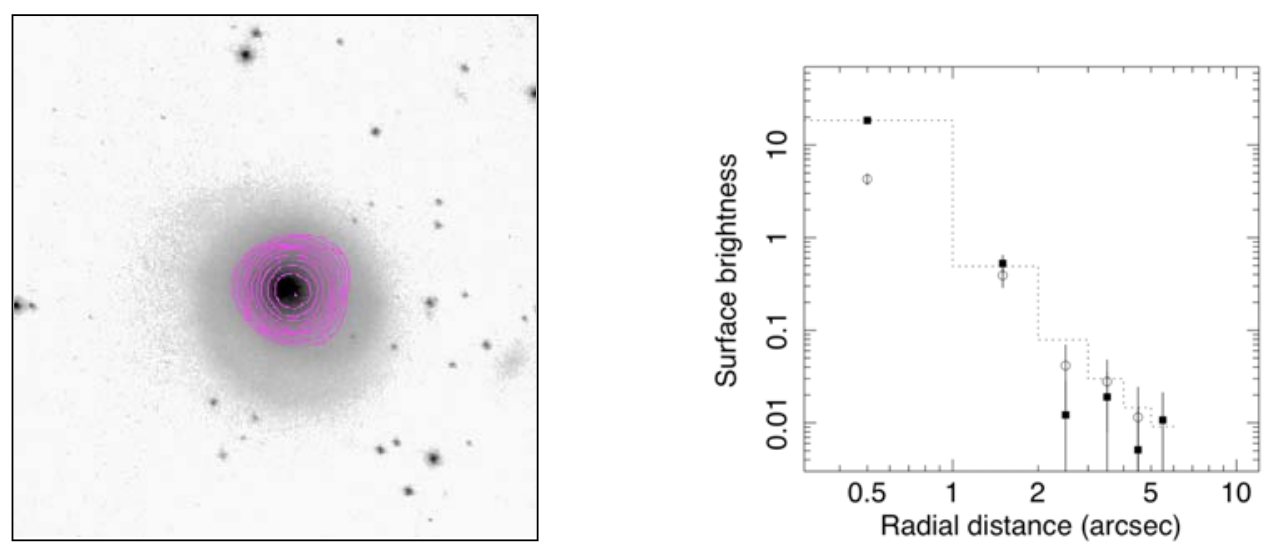

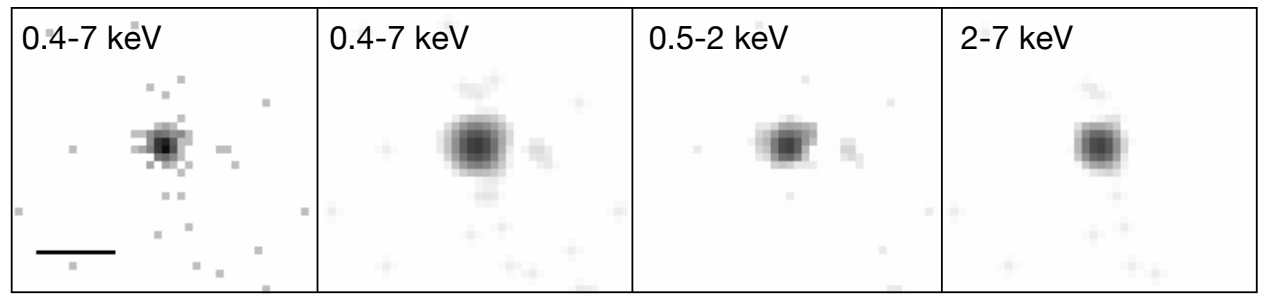

Fig. C.36. 


\section{CGCG448-020}
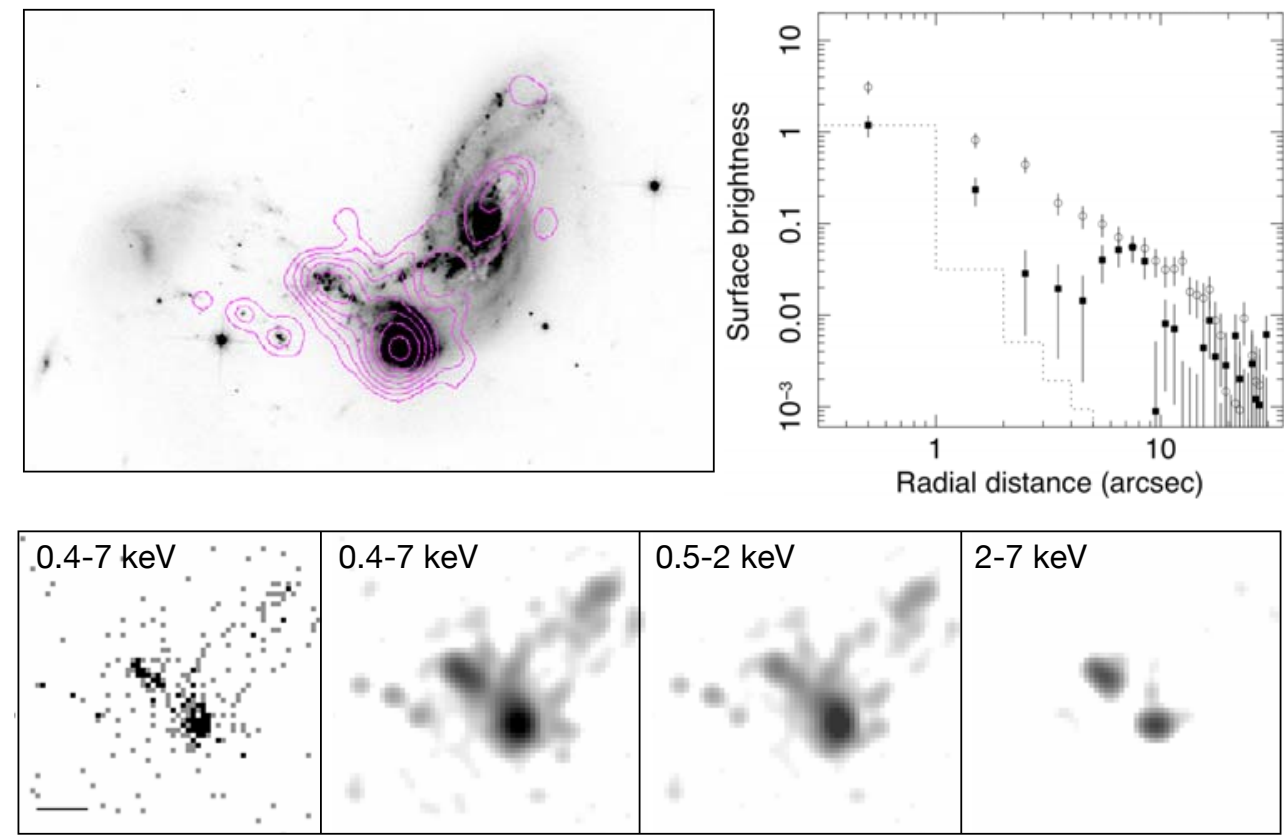

Fig. C.37.

\section{ESO 286-IG19}
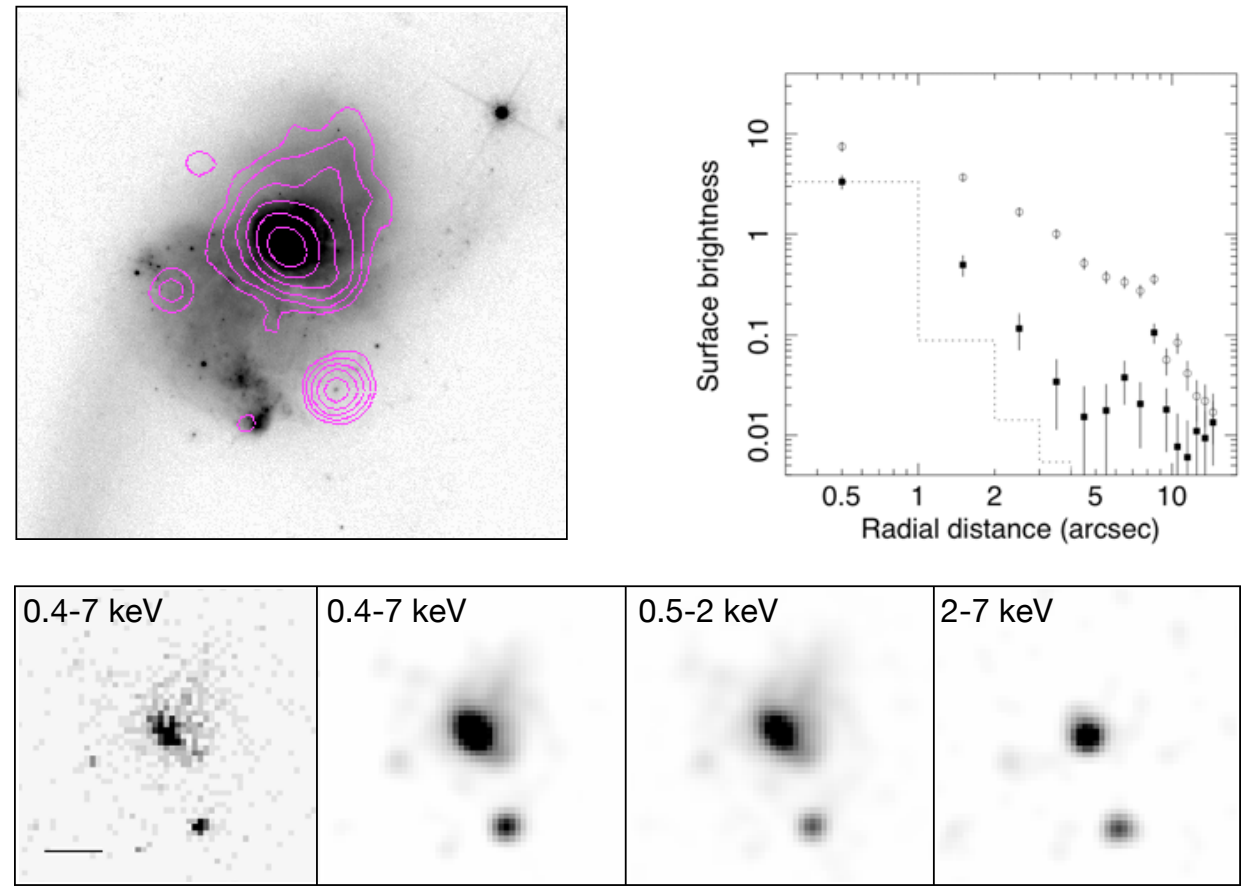

Fig. C.38. 


\section{IRAS $21101+5810$}
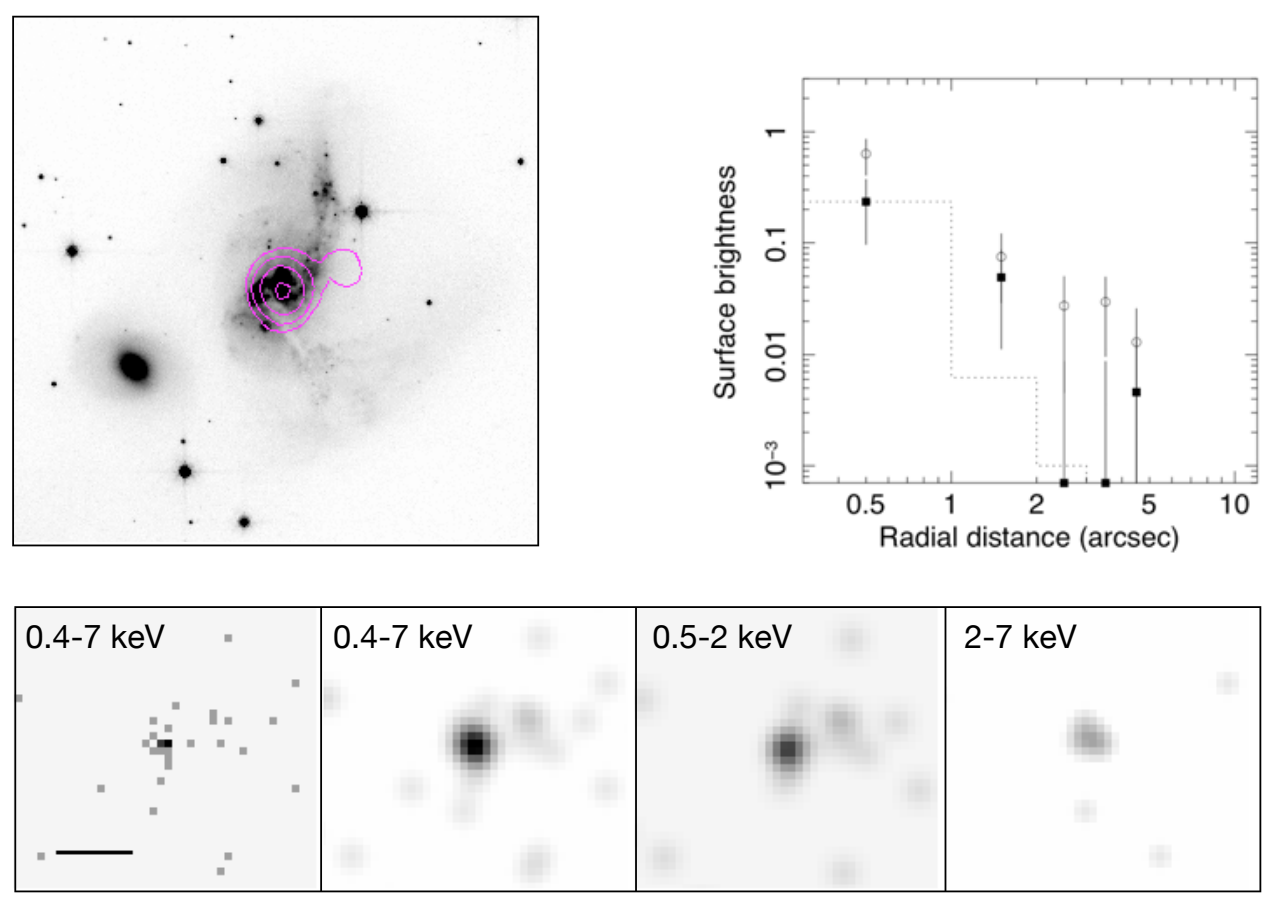

Fig. C.39.

\section{ESO 239-IG2}
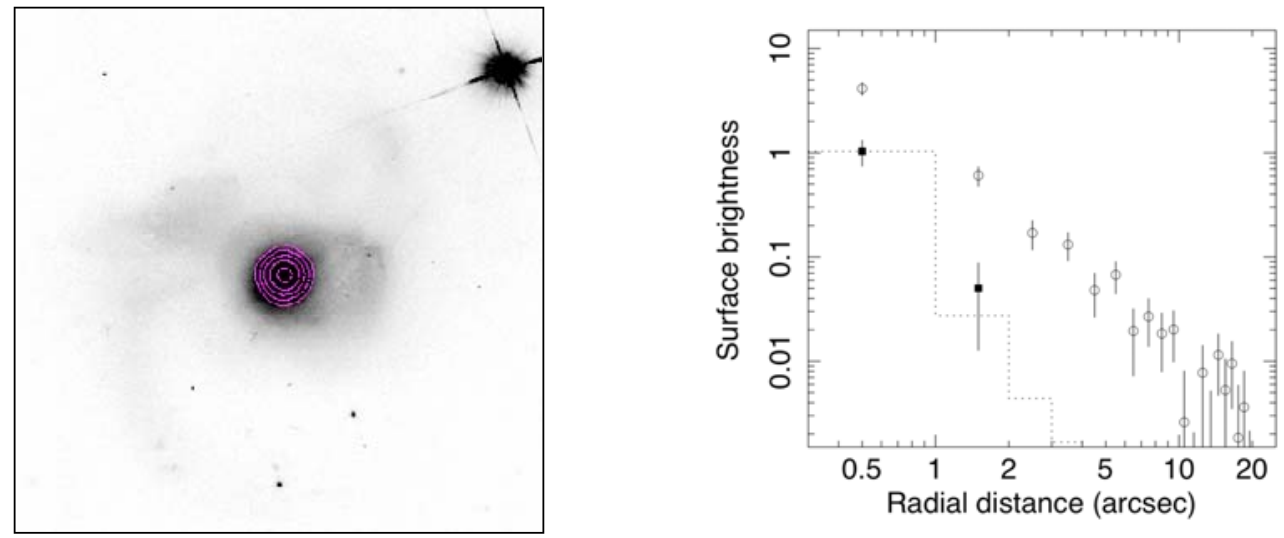

\begin{tabular}{|c|c|c|c|}
\hline $\begin{array}{c}0.4-7 \mathrm{keV} \\
\because \\
\vdots\end{array}$ & $=0.4-7 \mathrm{keV}$ & (2) & $2-7 \mathrm{keV}$ \\
\hline
\end{tabular}

Fig. C.40. 
F22491-1808
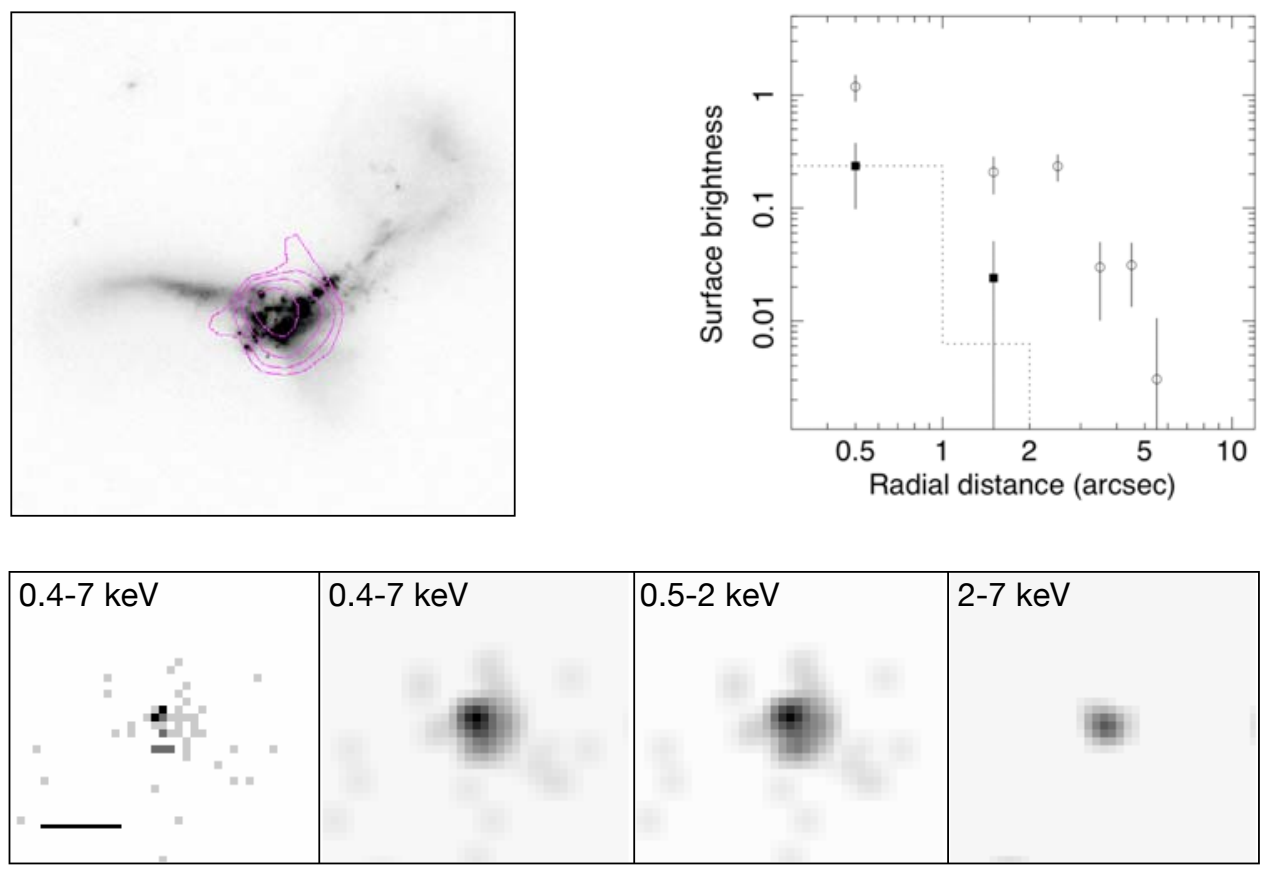

Fig. C.41.

\section{ESO 148-IG2}
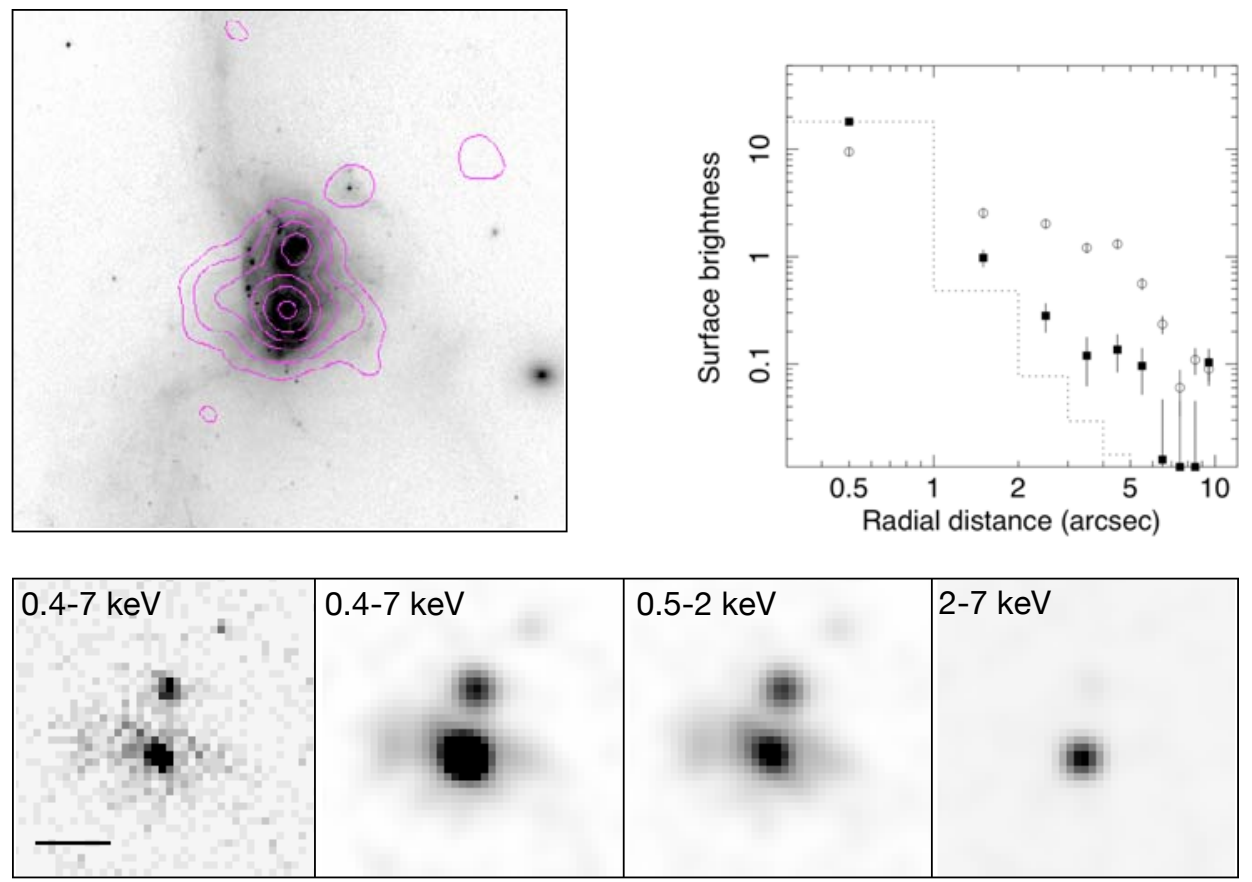

Fig. C.42. 


\section{ESO 77-IG14}
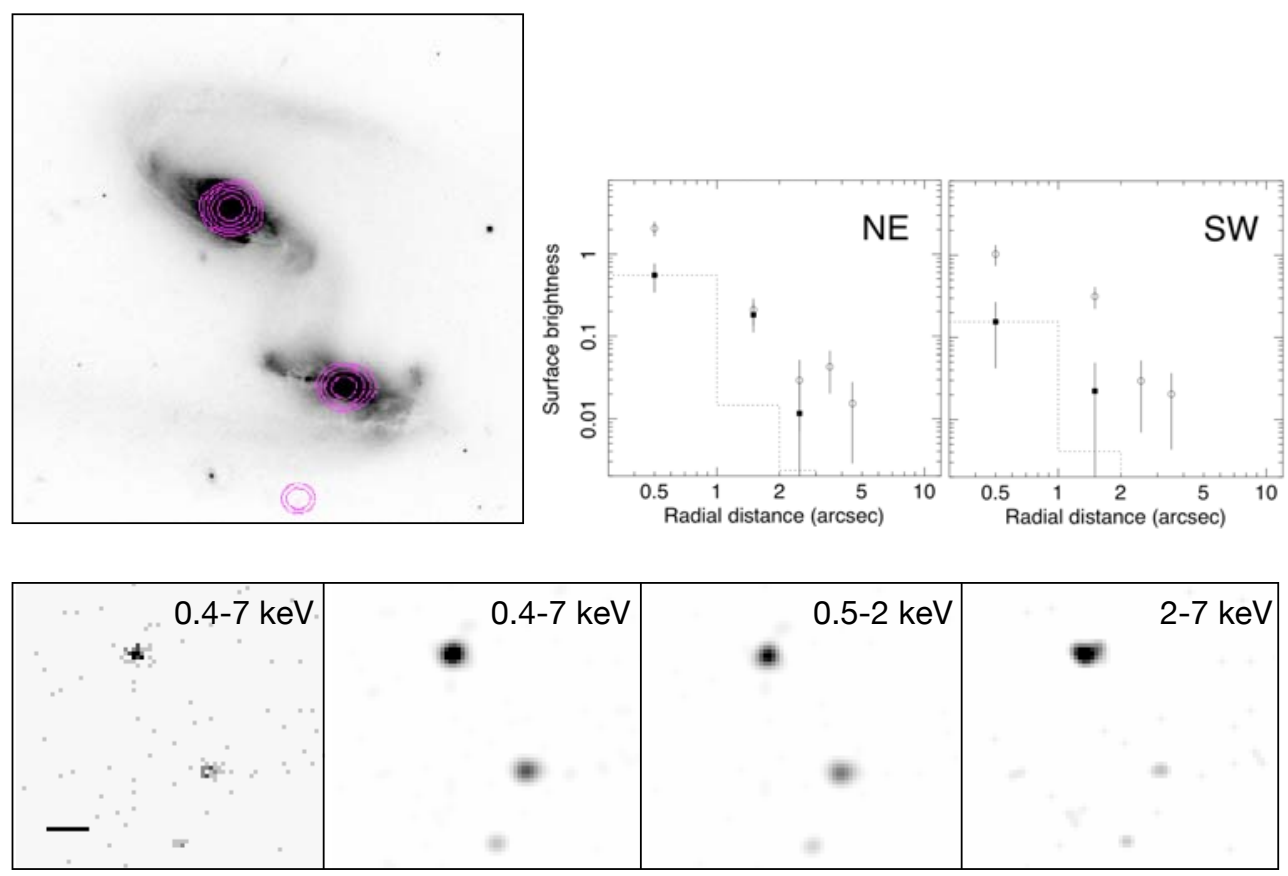

Fig. C.43.

\section{F23365+3604}
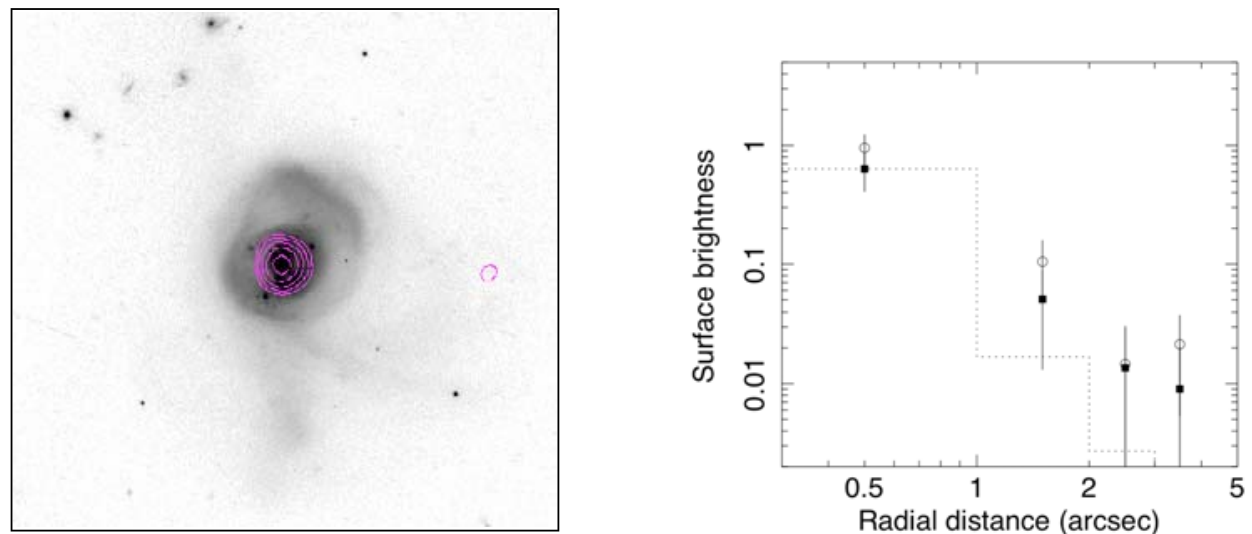

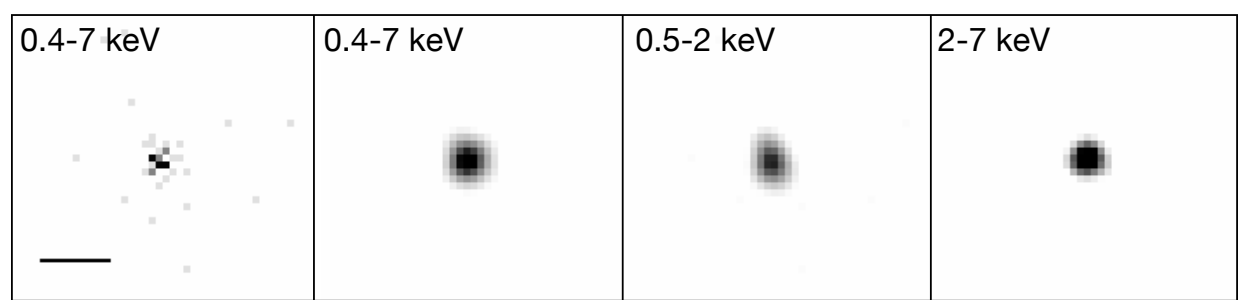

Fig. C.44. 